\title{
Multivariate Data Analysis with TMVA
}

\author{
Andreas Hoecker and Helge Voss \\ CERN, Switzerland and MPI-K Heidelberg, \\ Germany
}

\section{Introduction}

Multivariate data analysis (MVA) maps $n$ observed variables characterising an event species - also called the $n$-dimensional feature space - in, say, a physics experiment, onto a one or more dimensional output variable. In the case of multivariate regression this output variable directly represents the regression estimate, while for classification the output value corresponds to a decision boundary in the original $n$ dimensional feature space, which carries a particular "signal" efficiency and "background" rejection. One can hence choose the optimal working point in terms of signal efficiency and background rejection from the distribution of the MVA output values, along which cuts are defined.

If for a classification problem the probability density functions were known for the two different event classes (e.g. signal and background), the optimal mapping function, that is the one which for any selection efficiency achieves the best possible background rejection, would be given by the ratio of the probability density functions of the event classes. ${ }^{1}$ This is known as the Neyman-Pearsons Lemma [1]. Unfortunately however, the probability density functions are unknown in most applications. One may distinguish two different MVA approaches to deal with this ignorance : either one attempts to estimate the true underlying probability density functions, as for example done in nearest neighbour and multidimensional kernel methods, or one tries to model the optimal decision boundary in the feature space as done for example in neural networks.

These proceedings introduce the Toolkit for Multivariate Analysis (TMVA), a ROOT-integrated [2] software package for the processing, parallel evaluation and application of multivariate classification and multivariate regression techniques. All multivariate techniques in TMVA belong to the family of "supervised learnning" algorithms. They make use of training events, for which the desired output is known, to determine the mapping function that either discribes a decision boundary (classification) or an approximation of the underlying functional behaviour defining the target value (regression). The mapping function can contain various degrees of approximations and may be a single global function, or a set of local models. TMVA is specifically designed for the needs of high-energy physics (HEP) applications, but should not be restricted to these. The package includes :

- Rectangular cut optimisation (binary splits, Sec. 7.1).

- Projective likelihood estimation (Sec. 7.2).

- Multi-dimensional likelihood estimation (PDE range-search - Sec. 7.3, PDE-Foam - Sec. 7.4, and $\mathrm{k}-\mathrm{NN}$ - Sec. 7.5).

- Linear and nonlinear discriminant analysis (H-Matrix - Sec. 7.6, Fisher - Sec. 7.7, LD - Sec. 7.8, FDA - Sec. 7.9).

- Artificial neural networks (three different multilayer perceptron implementations - Sec. 7.10).

- Support vector machine (Sec. 7.11).

- Boosted/bagged decision trees (Sec. 7.12).

- Predictive learning via rule ensembles (RuleFit, Sec. 7.13).

\footnotetext{
${ }^{1}$ If the mapping function is known it also corresponds to the regression result.
} 
- A generic boost classifier allowing one to boost any of the above classifiers (Sec. 8).

- A generic category classifier allowing one to split the training data into disjoint categories with independent MVAs.

The software package consists of abstract, object-oriented implementations in $\mathrm{C}_{+}+/ \mathrm{ROOT}$ for each of these multivariate analysis (MVA) techniques, as well as auxiliary tools such as parameter fitting and transformations. It provides training, testing and performance evaluation algorithms and visualisation scripts. Detailed descriptions of all the TMVA methods and their options for classification and (where available) regression tasks are given in Sec. 7. Their training and testing is performed with the use of user-supplied data sets in form of ROOT trees or text files, where each event can have an individual weight. Preselection requirements and transformations (preprocessing) can be applied to input data. TMVA supports the use of variable combinations and formulas with a functionality similar to the one available for the Draw command of a ROOT tree.

TMVA guarantees an unbiased performance comparison between MVA methods : they all see the same training and test data, and are evaluated following the same prescriptions within the same execution job. Solely data preprocessing options can be individually invoked for different MVA methods, allowing also the comparison of one and the same algorithm on pre- and non-preprocessed data.

A Factory class organises the interaction between the user and the TMVA analysis steps. It performs preanalysis and preprocessing of the training data to assess basic properties of the discriminating variables used as inputs to the classifiers.

The linear correlation coefficients of the input variables are calculated and displayed. For regression, also nonlinear correlation measures are given, such as the correlation ratio and mutual information between input variables and output target. A preliminary variable ranking is derived, which is later superseded by algorithm-specific variable rankings. For classification problems, the variables can be linearly transformed (individually for each MVA method) into a non-correlated variable space, projected upon their principle components, or transformed into a normalised Gaussian shape. Transformations can also be arbitrarily concatenated.

To compare the signal-efficiency and background-rejection performance of the classifiers, or the average variance between regression target and estimation, the analysis job prints - among other criteria tabulated results for some benchmark values (see Sec. 2.1.3). Moreover, a variety of graphical evaluation information acquired during the training, testing and evaluation phases is stored in a ROOT output file. These results can be displayed using macros, which are conveniently executed via graphical user interfaces (each one for classification and regression) that come with the TMVA distribution (see Sec. 2.1.6).

The TMVA training job runs alternatively as a ROOT script, as a standalone executable, or as a python script via the PyROOT interface. Each MVA method trained in one of these applications writes its configuration and training results in a result ("weight") file, which in the default configuration has human readable XML format.

A light-weight Reader class is provided, which reads and interprets the weight files (interfaced by the corresponding method), and which can be included in any $\mathrm{C}++$ executable, ROOT macro, or python analysis job (see Sec. 2.2).

For standalone use of the trained MVA method, TMVA also generates lightweight $\mathrm{C}++$ response classes (not available for all methods), which contain the encoded information from the weight files so that these are not required anymore. These classes do not depend on TMVA or ROOT, neither on any other external library.

This article introduces typical multivariate classification and regression analyses with TMVA, including training optimisation, performance evaluation with indepndent test samples using the TMVA-specific evaluation tools, and the appliction to a data sample with unknown properties. We describe the preprocessing of the input data such as the transformation of input variables and event sorting (Sec. 3), introduce the optimisation and fitting tools commonly used by the methods (Sec. 5), and describe the basic principles of the TMVA methods. For a more general introduction to MVA techniques and their motivation we refer to the other contributions in these proceedings or excellent reviews on this subject (see, e.g., Refs. [3, 4, 5]). For a coding reference we refer to the TMVA Users Guide [6]. An attempt to provide guidance on which MVA method to use for varying problems and input conditions in Sec. 9. An overall summary of the implementation status of all TMVA methods is provided in Sec. 10. 
TMVA is an open source product, developed and maintained at Sourceforge.net (http://tmva.sourceforge.net). It is built upon ROOT (http://root.cern.ch/), hence for TMVA to run ROOT must be installed. Since ROOT version 5.11/06, TMVA is also an integral part of the ROOT distribution and can be invoked directly from the ROOT prompt.

\section{Multivariate Data Analysis with TMVA}

A typical TMVA classification or regression analysis consists of two independent phases : the training phase, where the multivariate methods are trained, tested and evaluated, and an application phase, where the chosen methods are applied to the concrete classification or regression problem they have been trained for. An overview of the code flow for these two phases are sketched in Fig. 1. The TMVA framework ensures equal treatment for an arbitrary number of booked methods, which all obey the same interface, allowing the user to compare their performance in a straightforward manner.

In the training phase, the communication of the user with the data sets and the MVA methods is performed via a Factory object, created at the beginning of the program. The TMVA Factory provides member functions to specify the training and test data sets (ROOT trees or text files, where the training events can carry weights ${ }^{2}$ ), to register the discriminating input (and - in case of regression - target) variables, and to book the multivariate methods. Subsequently the Factory calls for training, testing and the evaluation of the booked MVA methods. Specific result ("weight") files are created after the training phase by each booked MVA method.

The application of training results to a data set with unknown sample composition (classification) target value (regression) is governed by a Reader object. During initialisation, the user registers the input variables and books the MVA methods that were found to be the most appropriate after evaluating the training results. As booking argument, the name of the weight file is given. The weight file provides for each of the methods full and consistent configuration according to the training setup and results. Within the event loop, the input variables are updated for each event, and the MVA response values and, in some cases, errors are computed.

\subsection{Training and evaluation (using the TMVA Factory)}

The input events that are handed to the Factory are internally copied and split into one training and one test ROOT tree, unless explicit training and test trees are provided. This guarantees a statistically independent evaluation of the MVA algorithms based on the test sample. ${ }^{3}$ It is possible to apply selection requirements (cuts) upon the input events. The selection of the training and test samples from the source trees can be random, alternating (events are chosen in alternating turns for the training and test samples) or as blocks.

\subsubsection{Booking MVA methods}

All MVA methods are booked via the Factory by specifying the method's type, plus a unique name chosen by the user, and a set of specific configuration options encoded in a string qualifier. If the same method type is booked several times with different options (which is useful to compare different sets of configurations for optimisation purposes), the specified names must be different to distinguish the instances and their weight files. A booking example for the likelihood method is given in Code Example 1 below. Detailed descriptions of the configuration options are given in the corresponding tools and MVA sections of the TMVA Users Guide [6]. With the MVA booking the initialisation of the Factory is complete and no MVA-specific actions are left to do. The Factory takes care of the subsequent training, testing and evaluation of the MVA methods.

\footnotetext{
${ }^{2}$ In next-to-leading order Monte Carlo generators, events with (unphysical) negative weights may occur in some phase space regions. Such events are often troublesome to deal with, and it depends on the concrete implementation of the MVA method, whether or not they are treated properiy. A summary for all TMVA methods is given in Table 6 . In cases where a method does not properly treat events with negative weights, it is advisable to ignore such events for the training - but to include them in the performance evaluation to not bias the results.

${ }^{3}$ A fully unbiased training and evaluation requires at least three statistically independent, data sets. See comments in Footnote 5 on page 8 .
} 


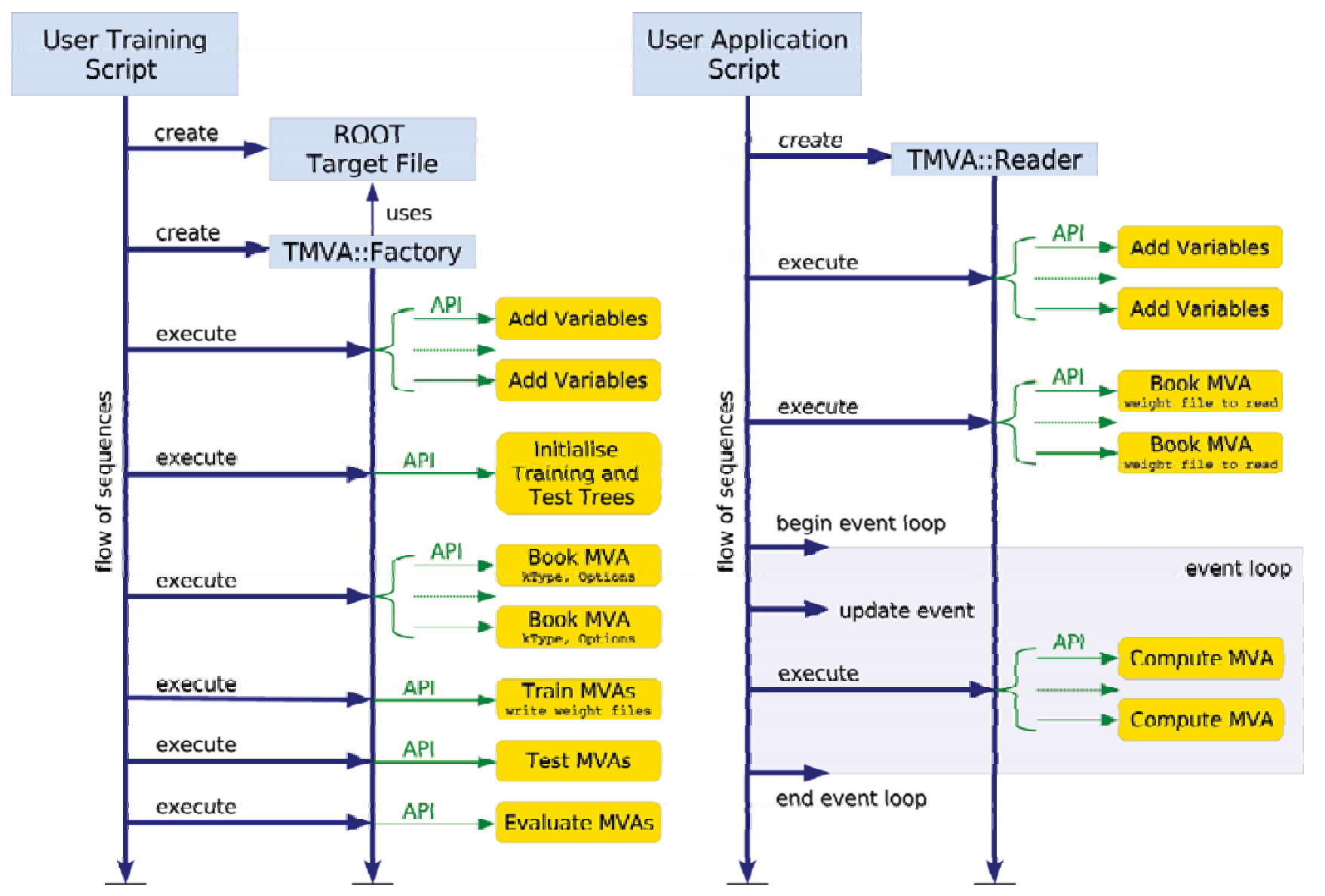

FIG. 1: Left : Flow (top to bottom) of a typical TMVA training application. The user script can be a ROOT macro: $\mathrm{C}++$ executable, python script or similar. The user creates a ROOT TFile, which is used by the TMVA Factory to store output histograms and trees. After creation by the user, the Factory organises the user's interaction with the TMVA modules. It is the only TMVA object directly created and owned by the user. First the discriminating variables that must be TFormula-compliant functions of branches in the training trees are registered. For regression also the target variable must be specified. Then, selected MVA methods are booked through a type identifier and a user-defined unique name, and configuration options are specified via an option string. The TMVA analysis proceeds by consecutively calling the training, testing and performance evaluation methods of the Factory. The training results for all booked methods are written to custom weight files in XML format and the evaluation histograms are stored in the output file. They can be analysed with specific macros that come with TMVA (cf. Tables 2 and 4 ).

Right : Flow (top to bottom) of a typical TMVA analysis application. The MVA methods qualified by the preceding training and evaluation step are now used to classify data of unknown signal and background composition or to predict a regression target. First, a Reader class object is created, which serves as interface to the method's response, just as was the Factory for the training and performance evaluation. The discriminating variables and references to locally declared memory placeholders are registered with the Reader. The variable names and types must be equal to those used for the training. The selected MVA methods are booked with their weight files in the argument, which fully configures them. The user then runs the event loop, where for each event the values of the input variables are copied to the reserved memory addresses, and the MVA response values (and in some cases errors) are computed. 


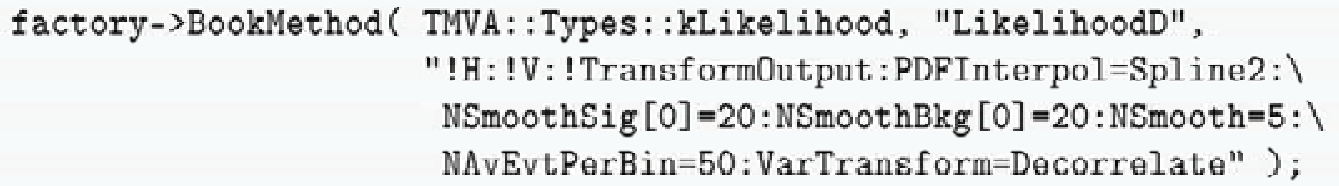

Code Example 1: Example booking of the likelihood method. The first argument is a unique type enumerator, the second is a unique user-defined name, and the third is a configuration option string that is specific to the method. For options that are not explicitly set in the string default values are used, which are printed to standard output. All specific options are explained in the tools and MVA sections of the TMVA Users Guide [6]. There is no difference in the booking of methods for classification or regression applications.

Upon request (option "H") the TMVA methods print concise help messages. These include a brief description of the algorithm, a performance assessment, and hints for setting the most important configuration options.

\subsubsection{Training and testing the MVA methods}

The training of the booked methods is invoked by the factory for all methods in one go. The training results together with the complete configuration information are stored in XML-formatted weight files.

The trained MVA methods are applied to the test data set and provide scalar outputs according to which an event can be classified as either signal or background, or which estimate the regression target. ${ }^{4}$ The MVA outputs are stored in a test tree to which a column is added for each booked method. The tree is eventually written to the output file and can be directly analysed in a ROOT session.

\subsubsection{Evaluating the MVA methods}

The Factory and data set classes of TMVA perform a preliminary property assessment of the input variables used by the MVA methods, such as computing correlation coefficients and ranking the variables according to their separation (for classification), or according to their correlations with the target variable(s) (for regression).

The performance measures differ between classification and regression problems. They are summarised below.

\section{Classification performance evaluation}

After training and testing, the linear correlation coefficients among the classifier outputs are printed. In addition, overlap matrices are derived (and printed) for signal and background that determine the fractions of signal and background events that are equally classified by each pair of classifiers. This is useful when two classifiers have similar performance, but a significant fraction of non-overlapping events. In such a case a combination of the classifiers (e.g., in a Committee classifier) could improve the performance (this can be extended to any combination of any number of classifiers).

The optimal method to be used for a specific analysis strongly depends on the problem at hand and no general recommendations can be given. To ease the choice TMVA computes a number of benchmark quantities that assess the performance of the methods on the independent test sample. For classification these are

- The signal efficiency at three representative background efficiencies (the efficiency is equal to 1 -rejection) obtained from a cut on the classifier output. Also given is the area of the background rejection versus signal efficiency function (the larger the area the better the performance).

\footnotetext{
${ }^{4}$ In classification mode, TMVA discriminates signal from background in data sets with unknown composition of these two samples. In frequent use cases the background (sometimes also the signal) consists of a variety of different populations with characteristic properties, which could call for classifiers with more than two discrimination classes. However, in practise it is uswally possible to serialise background fighting by training individual classifiers for each background source, and applying consecutive requirements to these. Since TMVA 4, the framework supports multi-class classification. However, the individual MVA methods have not yet been prepared for it.
} 
- The separation $\left\langle S^{2}\right\rangle$ of a classifier $y$, defined by the integral [9]

$$
\left\langle S^{2}\right\rangle=\frac{1}{2} \int \frac{\left(\hat{y}_{S}(y)-\hat{y}_{B}(y)\right)^{2}}{\hat{y}_{S}(y)+\hat{y}_{B}(y)} d y
$$

where $\hat{y}_{S}$ and $\hat{y}_{B}$ are the signal and background PDFs of $y$, respectively (cf. Sec. 2.1.5). The separation is zero for identical signal and background shapes, and it is one for shapes with no overlap.

- The discrimination significance of a classifier, defined by the difference between the classifier means for signal and background divided by the quadratic sum of their root-mean-squares.

The results of the evaluation are printed to standard output. Smooth background rejection/efficiency versus signal efficiency curves are written to the output ROOT file, and can be plotted using custom macros (see Sec. 2.1.6).

\section{Regression performance evaluation}

Ranking for regression is based on the correlation strength between the input variables or MVA method response and the regression target. Several correlation measures are implemented in TMVA to capture and quantify nonlinear dependencies. Their results are printed to standard output.

- The Correlation between two random variables $X$ and $Y$ is usually measured with the correlation coefficient $\rho$, defined by

$$
\rho(X, Y)=\frac{\operatorname{cov}(X, Y)}{\sigma_{X} \sigma_{Y}} .
$$

The correlation coefficient is symmetric in $X$ and $Y$, lies within the interval $[-1,1]$, and quantifies by definition a linear relationship. Thus $\rho=0$ holds for independent variables, but the converse is not true in general. In particular, higher order functional or non-functional relationships may not, or only marginally, be reflected in the value of $\rho$ (see Fig. 2).

- The correlation ratio is defined by

$$
\eta^{2}(Y \mid X)=\frac{\sigma_{E(Y \mid X)}}{\sigma_{Y}}
$$

where

$$
E(Y \mid X)=\int y P(y \mid x) d y
$$

is the conditional expectation of $Y$ given $X$ with the associated conditional probability density function $P(Y \mid X)$. The correlation ratio $\eta^{2}$ is in general not symmetric and its value lies within $[0,1]$, according to how well the data points can be fitted with a linear or nonlinear regression curve. Thus non-functional correlations cannot be accounted for by the correlation ratio. The following relations can be derived for $\eta^{2}$ and the squared correlation coefficient $\rho^{2}[11]$ :

$\circ \rho^{2}=\eta^{2}=1$, if $X$ and $Y$ are in a strict linear functional relationship.

○ $\rho^{2} \leq \eta^{2}=1$, if $X$ and $Y$ are in a strict nonlinear functional relationship.

○ $\rho^{2}=\eta^{2}<1$, if there is no strict functional relationship but the regression of $X$ on $Y$ is exactiy linear.

$\circ \rho^{2}<\eta^{2}<1$, if there is no strict functional relationship but some nonlinear regression curve is a better fit then the best linear fit.

Some characteristic examples and their corresponding values for $\eta^{2}$ are shown in Fig. 2. In the special case, where all data points take the same value, $\eta$ is undefined.

- Mutual information allows to detect any predictable relationship between two random variables. be it of functional or non-functional form. It is defined by [12]

$$
I(X, Y)=\sum_{X, Y} P(X, Y) \ln \frac{P(X, Y)}{P(X) P(Y)},
$$

where $P(X, Y)$ is the joint probability density function of the random variables $X$ and $Y$, and $P(X)$. $P(Y)$ are the corresponding marginal probabilities. Mutual information originates from information 

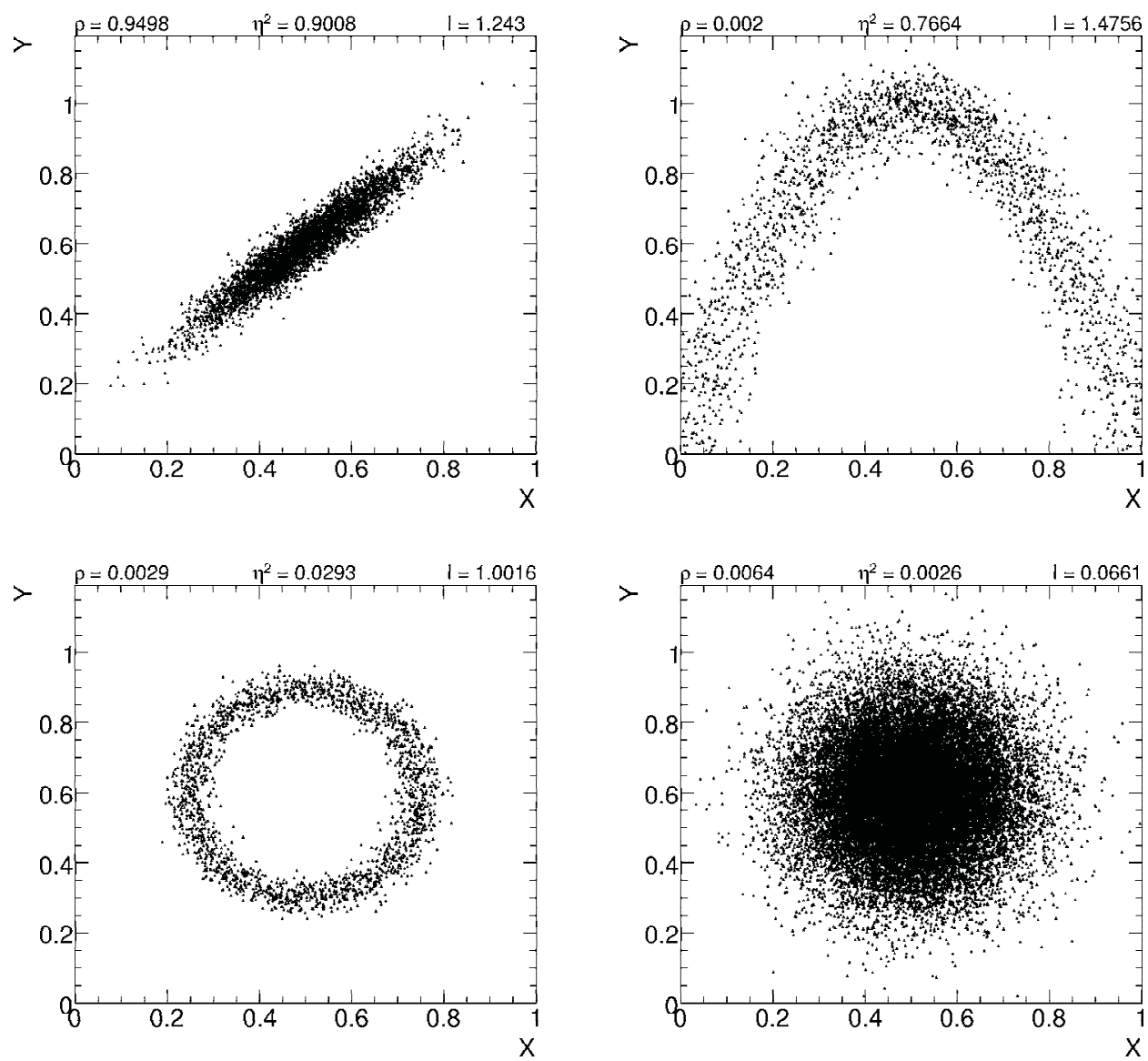

FIg. 2: Various types of correlations between two random variables and their corresponding values for the correlation coefficient $\rho$, the correlation ratio $\eta$, and mutual information $I$. Linear relationship (upper left), functional relationship (upper right), non-functional relationship (lower left), and independent variables (lower right).

theory and is closely related to entropy which is a measure of the uncertainty associated with a random variable. It is defined by

$$
H(X)=-\sum_{X} P(X) \ln P(X)
$$

where $X$ is the discrete random variable and $P(X)$ the associated probability density function. The connection between the two quantities is given by the following transformation

$$
\begin{aligned}
I(X, Y) & =\sum_{X, Y} P(X, Y) \ln \frac{P(X, Y)}{P(X) P(Y)} \\
& =\sum_{X, Y} P(X, Y) \ln \frac{P(X \mid Y)}{P_{X}(X)} \\
& =-\sum_{X, Y} P(X, Y) \ln P(X)+\sum_{X, Y} P(X, Y) \ln P(X \mid Y) \\
& =-\sum_{X, Y} P(X) \ln P(X)-\left(-\sum_{X, Y} P(X, Y) \ln P(X \mid Y)\right) \\
& =H(X)-H(X \mid Y),
\end{aligned}
$$

where $H(X \mid Y)$ is the conditional entropy of $X$ given $Y$. Thus mutual information is the reduction of the uncertainty in variable $X$ due to the knowledge of $Y$. Mutual information is symmetric and takes positive absolute values. In the case of two completely independent variables $I(X, Y)$ is zero. For experimental measurements the joint and marginal probability density functions are a priori unknown and must be approximated by choosing suitable binning procedures such as kernel estima- 


\begin{tabular}{llllllllllll}
\hline$\rho_{\text {PDF }}$ & 0.0 & 0.1 & 0.2 & 0.3 & 0.4 & 0.5 & 0.6 & 0.7 & 0.8 & 0.9 & 0.9999 \\
\hline$\rho$ & 0.006 & 0.092 & 0.191 & 0.291 & 0.391 & 0.492 & 0.592 & 0.694 & 0.795 & 0.898 & 1.0 \\
$\eta^{2}$ & 0.004 & 0.012 & 0.041 & 0.089 & 0.156 & 0.245 & 0.354 & 0.484 & 0.634 & 0.806 & 1.0 \\
$I$ & 0.093 & 0.099 & 0.112 & 0.139 & 0.171 & 0.222 & 0.295 & 0.398 & 0.56 & 0.861 & 3.071 \\
\hline
\end{tabular}

TAB. 1: Comparison of the correlation coefficient $\rho$, correlation ratio $\eta$, and mutual information $I$ for twodimensional Gaussian toy Monte-Carlo distributions with linear correlations as indicated (20000 data points/100 $\times$ 100 bins .

tion techniques (see, e.g., [13]). Consequently, the values of $I(X, Y)$ for a given data set will strongly depend on the statistical power of the sample and the chosen binning parameters.

For the purpose of ranking variables from data sets of equal statistical power and identical binning; however, we assume that the evaluation from a simple two-dimensional histogram without further smoothing is sufficient.

A comparison of the correlation coefficient $\rho$, the correlation ratio $\eta$, and mutual information $I$ for linearly correlated two-dimensional Gaussian toy MC simulations is shown in Table 1.

\subsubsection{Overtraining}

Overtraining occurs when a machine learning problem has too few degrees of freedom, because too many model parameters of an algorithm were adjusted to too few data points. The sensitivity to overtraining therefore depends on the MVA method. For example, a Fisher (or linear) discriminant can hardly ever be overtrained, whereas, without the appropriate counter measures, boosted decision trees usually suffer from at least partial overtraining, owing to their large number of nodes. Overtraining leads to a seeming increase in the classification or regression performance over the objectively achievable one, if measured on the training sample, and to an effective performance decrease when measured with an independent test sample. A convenient way to detect overtraining and to measure its impact is therefore to compare the performance results between training and test samples. Such a test is performed by TMVA with the results printed to standard output.

Various method-specific solutions to counteract overtraining exist. For example, binned likelihood reference distributions are smoothed before interpolating their shapes, or unbinned kernel density estimators smear each training event before computing the PDF; neural networks steadily monitor the convergence of the error estimator between training and test samples ${ }^{5}$ suspending the training when the test sample has passed its minimum; the number of nodes in boosted decision trees can be reduced by removing insignificant ones ("tree pruning"), etc.

\subsubsection{Other representations of MVA outputs for classification : probabilities and Rarity}

In addition to the MVA response value $y$ of a classifier, which is typically used to place a cut for the classification of an event as either signal or background, or which could be used in a subsequent likelihood fit, TMVA also provides the classifier's signal and background PDFs, $\hat{y}_{S(B)}$. The PDFs can be used to derive classification probabilities for individual events, or to compute any kind of transformation of which the Rarity transformation is implemented in TMVA.

- Classification probability : The techniques used to estimate the shapes of the PDFs are those developed for the likelihood classifier and can be customised individually for each method (the control options are given in Sec. 7). The probability for event $i$ to be of signal type is given by,

$$
P_{S}(i)=\frac{f_{S} \cdot \hat{y}_{S}(i)}{f_{S} \cdot \hat{y}_{S}(i)+\left(1-f_{S}\right) \cdot \hat{y}_{B}(i)},
$$

\footnotetext{
${ }^{5}$ Proper training and validation requires three statistically independent data sets : one for the parameter optimisation. another one for the overtraining detection, and the last one for the performance validation. In TMVA, the last two samples have been merged to increase statistics. The (usually insignificant) bias introduced by this on the evaluation results does not affect the analysis as far as classification cut efficiencies or the regression resolution are independently validated with data.
} 

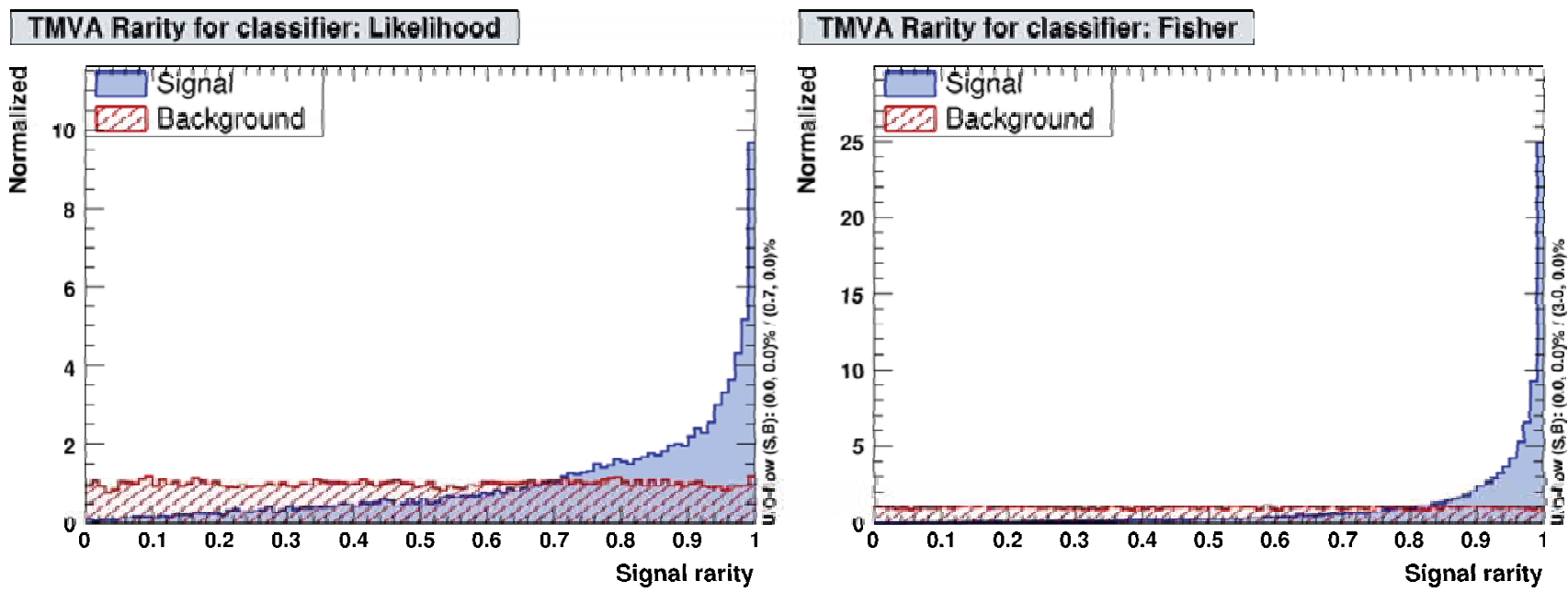

Fig. 3: Example plots for classifier Rarity distributions for signal and background events from the academic test sample. Shown are likelihood (left) and Fisher (right).

where $f_{S}=N_{S} /\left(N_{S}+N_{B}\right)$ is the expected signal fraction, and $N_{S(B)}$ is the expected number of signal (background) events (default is $\left.f_{S}=0.5\right){ }^{6}$

- Rarity : The Rarity $\mathcal{R}(y)$ of a classifier $y$ is given by the integral [10]

$$
\mathcal{R}(y)=\int_{-\infty}^{y} \hat{y}_{B}\left(y^{\prime}\right) d y^{\prime},
$$

which is defined such that $\mathcal{R}\left(y_{B}\right)$ for background events is uniformly distributed between 0 and 1 , while signal events cluster towards 1 . The signal distributions can thus be directly compared among the various classifiers. The stronger the peak towards 1 , the better is the discrimination. Another useful aspect of the Rarity is the possibility to directly visualise deviations of a test background (which could be physics data) from the training sample, by exhibition of non-uniformity.

The Rarity distributions of the Likelihood and Fisher classifiers for the present example are plotted in Fig. 3. Since Fisher performs better (cf. Fig. 7 on page 15), its signal distribution is stronger peaked towards 1. By construction, the background distributions are uniform within statistical fluctuations.

The probability and Rarity distributions can be plotted with dedicated macros, invoked through corresponding GUI buttons.

\subsubsection{ROOT macros to plot training, testing and evaluation results}

TMVA provides simple GUIs (see Fig. ??), which interface ROOT macros that visualise the various steps of the training analysis. They are described in Tables 2 and 4.

Examples for plots produced by these macros are given in Figs. 5-7 for a classification problem. The distributions of the input variables for signal and background according to our example job are shown in Fig. 4. It is useful to quantify the correlations between the input variables. These are drawn in form of a scatter plot with the superimposed profile for two of the input variables in Fig. 5 (upper left). As will be discussed in Sec. 3, TMVA allows to perform a linear decorrelation transformation of the input variables prior to the MVA training (for classification only). The result of such decorrelation is shown at the upper right hand plot of Fig. 5. The lower plots display the linear correlation coefficients between all input variables, for the signal and background training samples of the classification example.

Figure 6 shows several classifier output distributions for signal and background events based on the test sample. By TMVA convention, signal (background) events accumulate at large (small) classifier output values. Hence, cutting on the output and retaining the events with $y$ larger than the cut requirement

${ }^{6}$ The $P_{S}$ distributions may exhibit a somewhat peculiar structure with frequent narrow peaks. They are generated by regions of classificr output values in which $\hat{y}_{S} \propto \hat{y}_{B}$ for which $P_{S}$ becomes a constant. 


\begin{tabular}{|c|c|}
\hline Macro & Description \\
\hline variables.C & $\begin{array}{l}\text { Plots the signal and background MVA input variables (training } \\
\text { sample). }\end{array}$ \\
\hline correlationscatter.C & $\begin{array}{l}\text { Plots superimposed scatters and profiles for all pairs of input variables } \\
\text { used during the training phase (separate plots for signal and back- } \\
\text { ground in case of classification). }\end{array}$ \\
\hline correlations.C & $\begin{array}{l}\text { Plots the linear correlation matrices for the input variables in the trai- } \\
\text { ning sample (distinguishing signal and background for classification). }\end{array}$ \\
\hline mvas.C & $\begin{array}{l}\text { Plots the classifier response distributions of the test sample for signal } \\
\text { and background. The second argument allows to also plot the pro- } \\
\text { bability (1) and Rarity (2) distributions of the classifiers, as well as } \\
\text { a comparison of the output distributions between test and training } \\
\text { samples. }\end{array}$ \\
\hline mvaeffs.C & $\begin{array}{l}\text { Signal and background efficiencies, obtained from cutting on the classi- } \\
\text { fier outputs, versus the cut value. Also shown are the signal purity and } \\
\text { the signal efficiency times signal purity corresponding to the expected } \\
\text { number of signal and background events before cutting (numbers gi- } \\
\text { ven by uscr). The optimal cuts according to the best significance are } \\
\text { printed on standard output. }\end{array}$ \\
\hline efficiencies.C & $\begin{array}{l}\text { Background rejection or background efficiency, versus signal efficiency } \\
\text { for the classifiers (test sample). The efficiencies are obtained by cutting } \\
\text { on the classifier outputs. This is traditionally the best plot to assess } \\
\text { the overall discrimination performance (ROC curve). }\end{array}$ \\
\hline paracoor.C & $\begin{array}{l}\text { Draws diagrams of "Parallel coordinates" [33] for signal and back- } \\
\text { ground, used to visualise the correlations among the input variables, } \\
\text { but also between the MVA output and input variables (indicating the } \\
\text { importance of the variables). }\end{array}$ \\
\hline
\end{tabular}

TAB. 2: ROOT macros for the representation of the TMVA input variables and classification results. The macros are conveniently called via a GUI. Macros for the representation of regression results are given in Table 3 . Plotting macros for MVA method specific information are listed in Table 4.

\begin{tabular}{|ll|}
\hline Macro & Description \\
\hline deviations.C & $\begin{array}{l}\text { Plots the linear deviation between regression target value and MVA } \\
\text { response or input variables for test and training samples. }\end{array}$ \\
regression_averagedevs.C C $\begin{array}{l}\text { Draws the average deviation between the MVA output and the regres- } \\
\text { sion target value for all trained methods. }\end{array}$ \\
\hline
\end{tabular}

TAB. 3: ROOT macros for the representation of the TMVA regression results called via the regression GUI.

selects signal samples with efficiencies and purities that respectively decrease and increase with the cut value. The resulting relations between background rejection versus signal efficiency are shown in Fig. 7 for all classifiers that were used in the example macro. This plot belongs to the class of Receiver Operating Characteristic (ROC) diagrams, which in its standard form shows the true positive rate versus the false positive rate for the different possible cutpoints of a hypothesis test.

As an example for multivariate regression, Fig. 8 displays the deviation between the regression output and target values for linear and nonlinear regression algorithms.

More macros are available to validate training and response of specific MVA methods. For example, the macro likelihoodrefs. C compares the probability density functions used by the likelihood classifier to the normalised variable distributions of the training sample. It is also possible to visualize the MLP neural network architecture and to draw decision trees (see Table 4). 


\begin{tabular}{|c|c|}
\hline Macro & Description \\
\hline likelihoodrefs.C & $\begin{array}{l}\text { Plots the reference PDFs of all input variables for the projective likeli- } \\
\text { hood method and compares it to original distributions obtained from } \\
\text { the training sample. }\end{array}$ \\
\hline network.C & $\begin{array}{l}\text { Draws the TMVA-MLP architecture including weights after training } \\
\text { (does not work for the other ANNs). }\end{array}$ \\
\hline annconvergencetest.C & $\begin{array}{l}\text { Plots the MLP error-function convergence versus the training epoch } \\
\text { for training and test events (does not work for the other ANNs). }\end{array}$ \\
\hline BDT.C (i) & Draws the ith decision tree of the trained forest. \\
\hline BDTControlPlots.C & $\begin{array}{l}\text { Plots distributions of boost weights throughout forest, boost weights } \\
\text { versus decision tree, error fraction, number of nodes before and after } \\
\text { pruning and the coefficient } \alpha \text {. }\end{array}$ \\
\hline mvarefs.C & $\begin{array}{l}\text { Plots the PDFs used to compute the probability response for a classi- } \\
\text { fier, and compares it to the original distributions. }\end{array}$ \\
\hline PlotFoams.C & $\begin{array}{l}\text { Draws the signal and background foams created by the method PDE- } \\
\text { Foam. }\end{array}$ \\
\hline rulevis.C & $\begin{array}{l}\text { Plots the relative importance of rules and linear terms. The } 1 \mathrm{D} \text { plots } \\
\text { show the accumulated importance per input variable. The } 2 \mathrm{D} \text { scat- } \\
\text { ter plots show the same but correlated between the input variables } \\
\text { These plots help to identify regions in the parameter space that are } \\
\text { important for the model. }\end{array}$ \\
\hline
\end{tabular}

TAB. 4: List of ROOT macros representing results for specific MVA methods. The macros require that these methods have been included in the training. All macros take as first argument the name of the ROOT file containing the histograms.

\subsection{Applying the MVAs (using the TMVA Reader)}

After training and evaluation, the most performing MVA methods are chosen and used to classify events in data samples with unknown signal and background composition, or to predict values of a regression target. Analogously to the Factory, the communication between the user application and the MVA methods is interfaced by the TMVA Reader.

The user registers the names of the input variables with the Reader and the selected MVA methods are booked using the weight files from the preceding training job. Within the event loop, the response value of a classifier, and - if available - its error, for a given set of input variables provided by the user. are obtained from the Reader via the method's unique name identifier.

The output of a classifier may then be used for example to put a cut that increases the signal purity of the sample (the achievabie purities can be read off the evaluation results obtained during the test phase), or it could enter a subsequent maximum-likelihood fit, or similar. The error reflects the uncertainty, which may be statistical, in the output value as obtained from the training information.

For regression, multi-target response is already supported in TMVA, so that the Reader returns a tuple of regression estimates for each input event. The output of a regression method could be directly used for example as energy estimate for a calorimeter cluster as a function of the cell energies.

Instead of the classifier response values, one may also retrieve the ratio (12) from the Reader, which, if properly normalised to the expected signal fraction in the sample, corresponds to a probability. Similarly, the Rarity (13) of a classifier can be retrieved.

To simplify the portability of the trained MVA response to any application the TMVA methods generate after the training lightweight standalone $\mathrm{C}++$ response classes including in the source code the content of the weight files. ${ }^{7}$ These classes do not depend on ROOT, neither on any other non-standard

\footnotetext{
7 At present, the class making functionality has been implemented for all MVA methods with the exception of cut optimisation, PDE-RS, PDE-Foam and k-NN. While for the former classifier the cuts can be easily implemented into the user application, and do not require an extra class, the implementation of a response class for PDE-RS or $k-N N$ requires a
} 

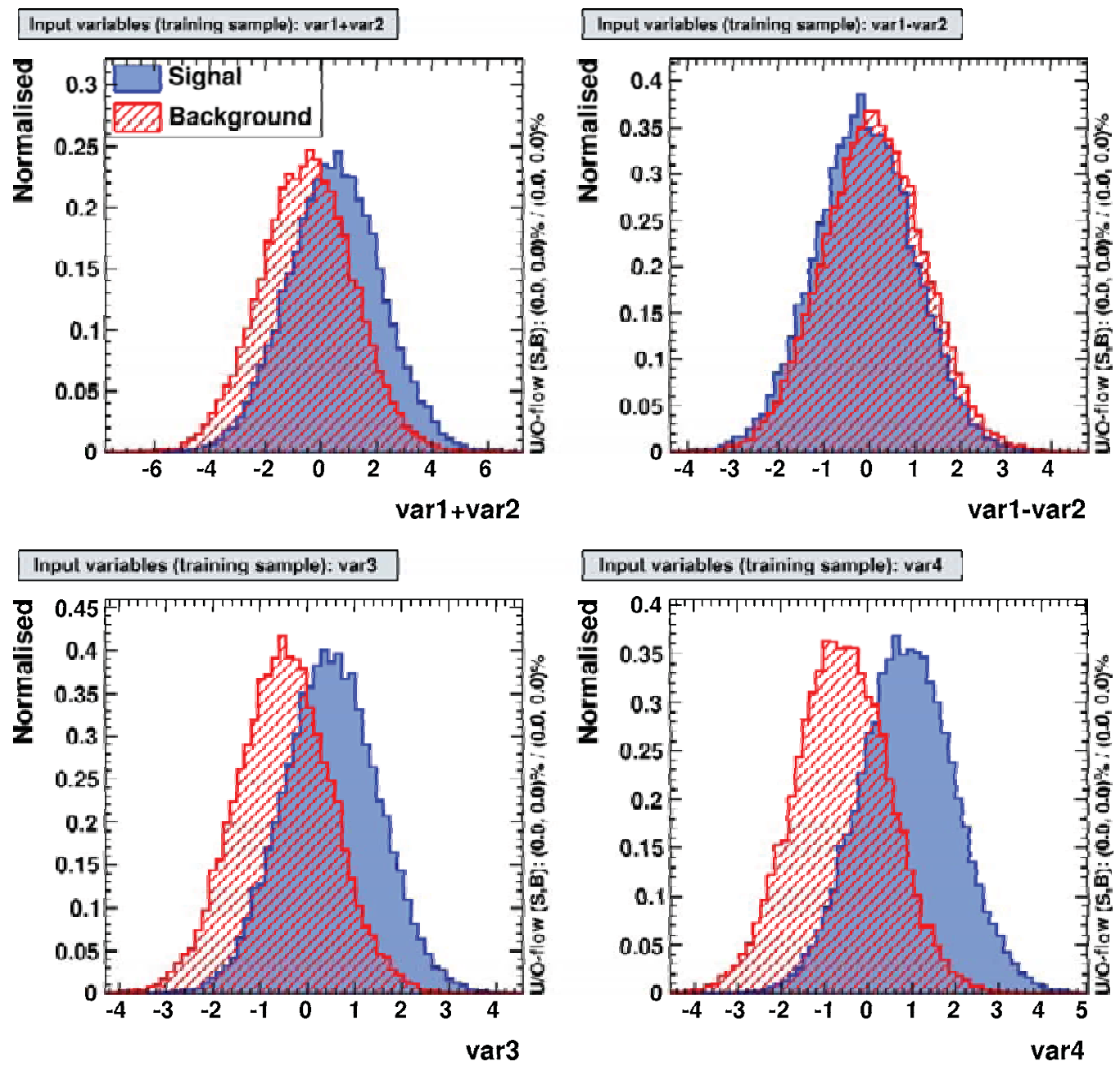

Fig. 4: Example plots for input variable distributions. The histogram limits are chosen to zoom into the bulk of the distributions, which may lead to truncated tails. The vertical text on the right-hand side of the plots indicates the under- and overflows.

library. They are presently only available for classification.

\section{Data Preprocessing}

It is possible to preprocess the discriminating input variables or the training events prior to presenting them to a multivariate method. Preprocessing can be useful to reduce correlations among the variables, to transform their shapes into more appropriate forms, or to accelerate the response time of a method (event sorting).

The preprocessing is completely transparent to the MVA methods. Any preprocessing performed for the training is automatically performed in the application through the Reader class. All the required information is stored in the weight files of the MVA method. Most preprocessing methods discussed below are only available for classification. An exception is the normalisation transformation, which exists for both classification and regression.

\subsection{Transforming input variables}

Currently four preprocessing transformations are implemented in TMVA :

- variable normalisation;

copy of the entire analysis code, which we have not attempted so far. We also point out that the use of the standalone $\mathrm{C}++$ class for BDT is not practical due to the colossal size of the generated code. 

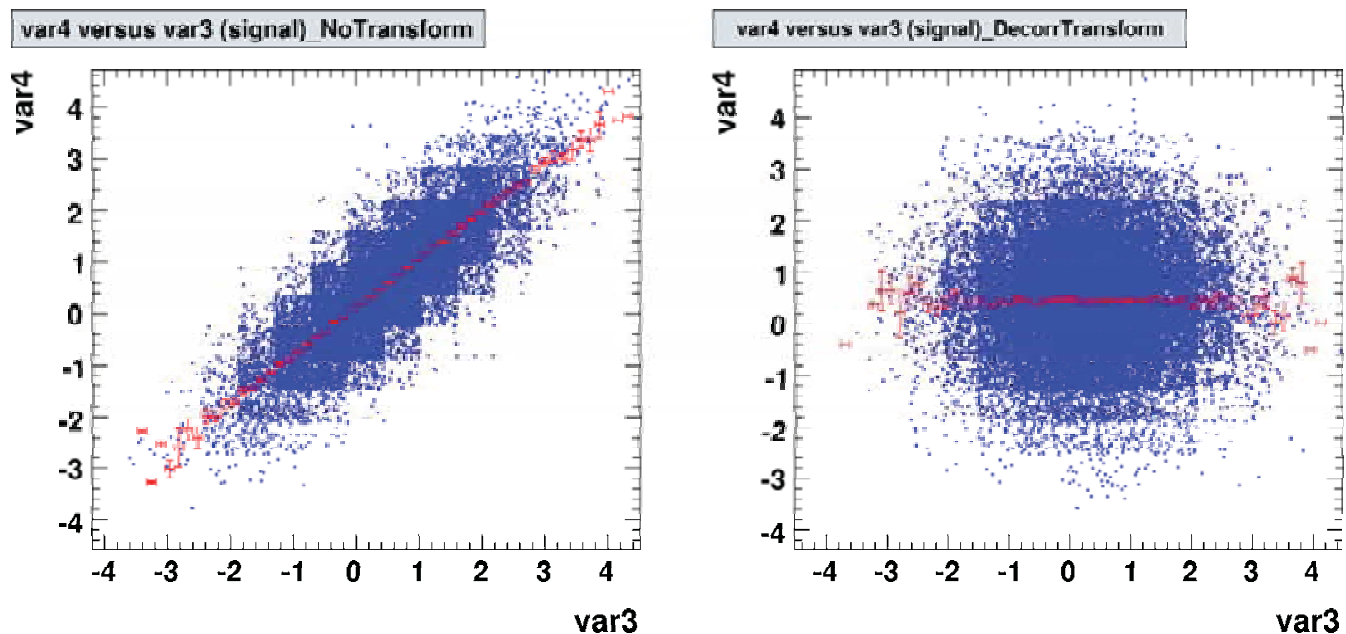

\section{Correlation Matrix (signal)}
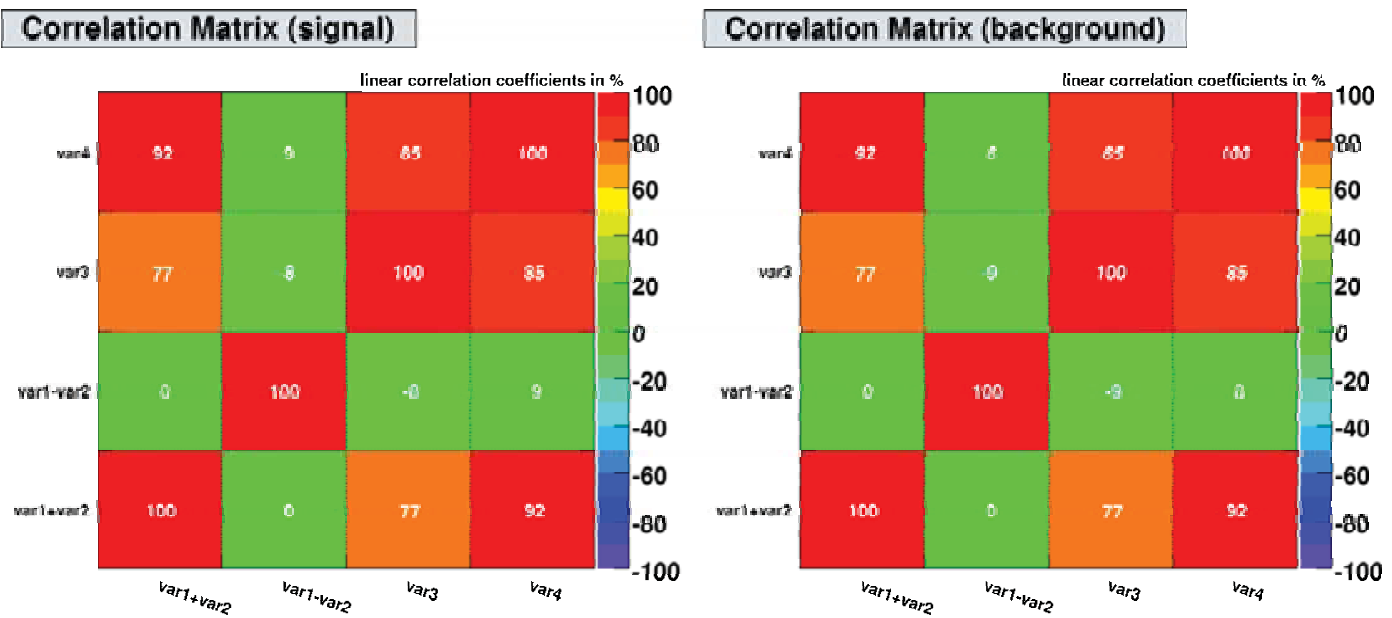

Fig. 5: Correlation between input variables. Upper left : correlations between var3 and var4 for the signal training sample. Upper right : the same after applying a linear decorrelation transformation (see Sec. 3.1.2). Lower plots : linear correlation coefficients for the signal and background training samples.

- decorrelation via the square-root of the covariance matrix;

- decorrelation via a principal component decomposition;

- transformation of the variables into Gaussian distributions ("Gaussianisation").

With the exception of normalisation, which exists for both classification and regression, the other preprocessing methods are currently only available for classification.

Technically, any transformation of the input variables is performed "on the fly" when the event is requested for training, testing or application. The preprocessing is hence fully transparent to the MVA methods. Any preprocessing performed for the training is automatically also performed in the application through the Reader class. All the required information is stored in the weight files of the MVA method.

\subsubsection{Variable normalisation}

Minimum and maximum values for all input variables are determined from the training events and used to linearly scale the input variables to lie within $[-1,1]$. Such a transformation is useful to allow direct comparisons between MVA weights applied to the variables. Large absolute weights may indicate strong separation power. Normalisation may also render minimisation processes, such as the adjustment of neural network weights, more effective.

\subsubsection{Variable decorrelation}

A drawback of, for example, the projective likelihood classifier (see Sec. 7.2) is that it ignores correlations among the discriminating input variables. Because in most realistic use cases this is not an accurate 

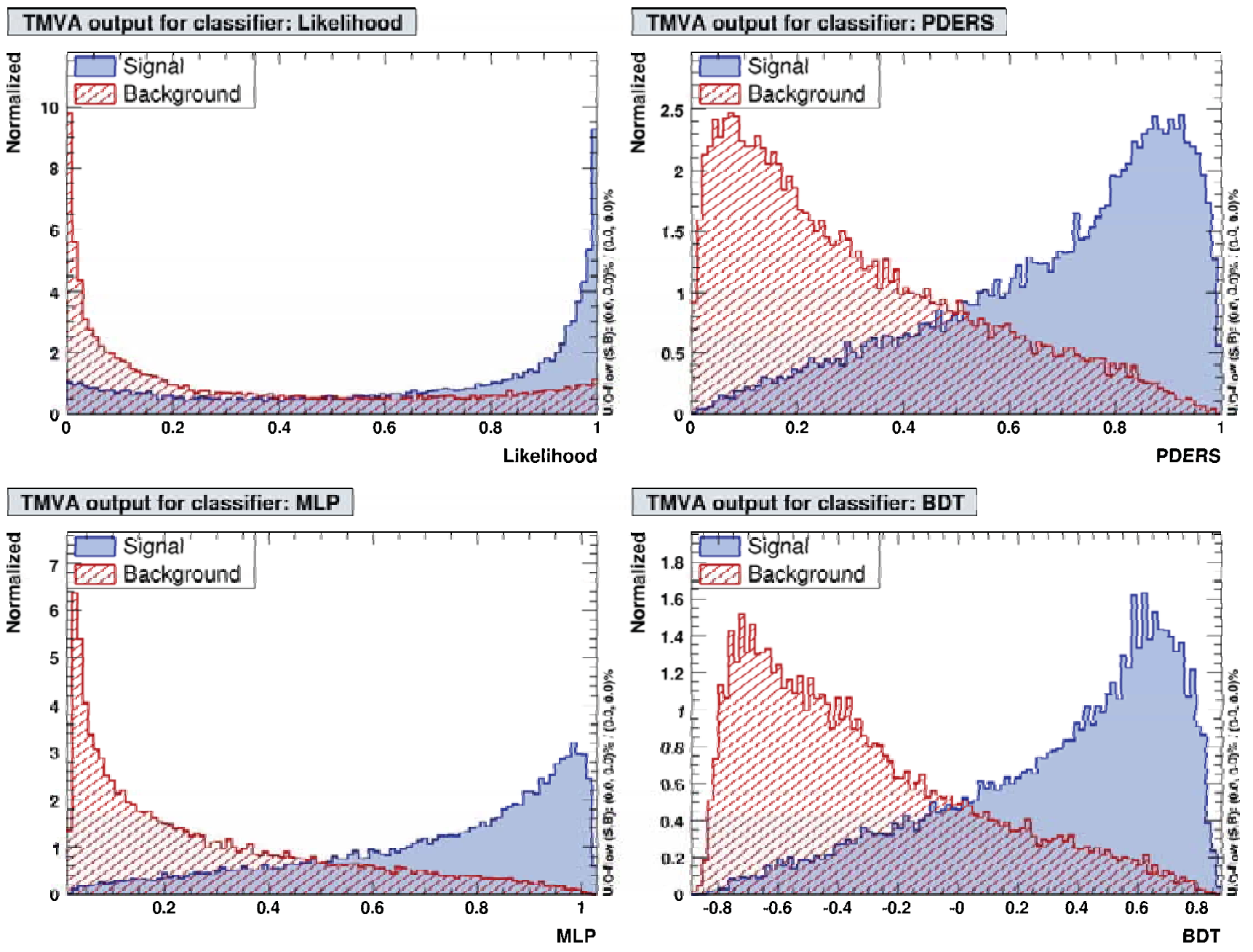

FIG. 6: Example plots for classifier output distributions for signal and background events from the academic test sample. Shown are likelihood (upper left), PDE range search (upper right), Multilayer perceptron (MLP - lower left) and boosted decision trees.

conjecture it leads to performance loss. Also other classifiers, such as rectangular cuts or decision trees, and even multidimensional likelihood approaches underperform in presence of variable correlations.

Linear correlations, measured in the training sample, can be taken into account in a straightforward manner through computing the square-root of the covariance matrix. The square-root of a matrix $C$ is the matrix $C^{\prime}$ that multiplied with itself yields $C: C=\left(C^{\prime}\right)^{2}$. TMVA computes the square-root matrix by means of diagonalising the (symmetric) covariance matrix

$$
D=S^{T} C S \quad \Rightarrow \quad C^{\prime}=S \sqrt{D} S^{T}
$$

where $D$ is a diagonal matrix, and where the matrix $S$ is symmetric. The linear decorrelation of the input variables is then obtained by multiplying the initial variable tuple $\mathbf{x}$ by the inverse of the square-root matrix

$$
\mathbf{x} \mapsto\left(C^{\prime}\right)^{-1} \mathbf{x}
$$

The transformations are typically different for signal and background events, because their correlation patterns usually differ. By default, in TMVA the transformations are computed using all training events, i.e. a collection of signal and background events. It is however possible to specify the use of a specific class only (e.g. Signal, Background), and this transformation will then be applied for all events. Different transformations for signal and background events are only useful for methods that explicitly distinguish signal and background hypotheses. This is the case for the likelihood and PDE-RS classifiers, where this is also exploited by default. For all other methods the user must choose which transformation to use.

The decorrelation is complete only for linearly correlated and Gaussian distributed variables. In real- 


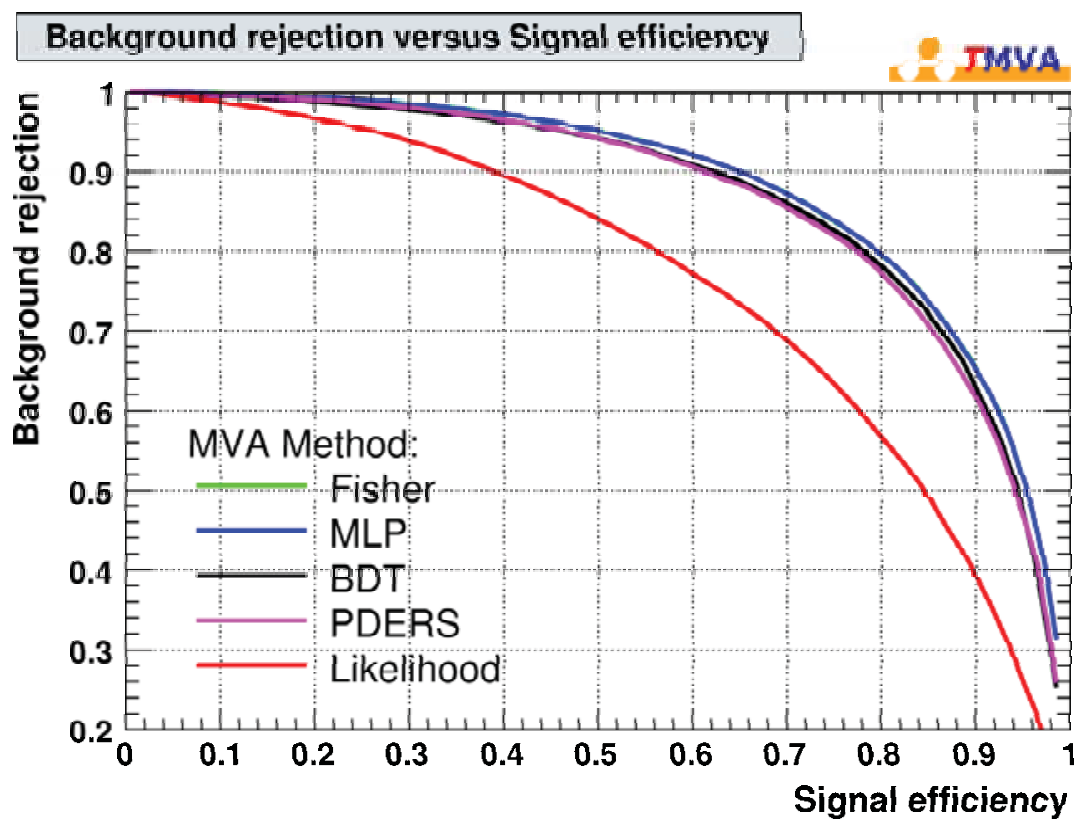

FIG. 7: Example for the background rejection versus signal efficiency ("ROC curve") obtained by cutting on the classifier outputs for the events of the test sample.
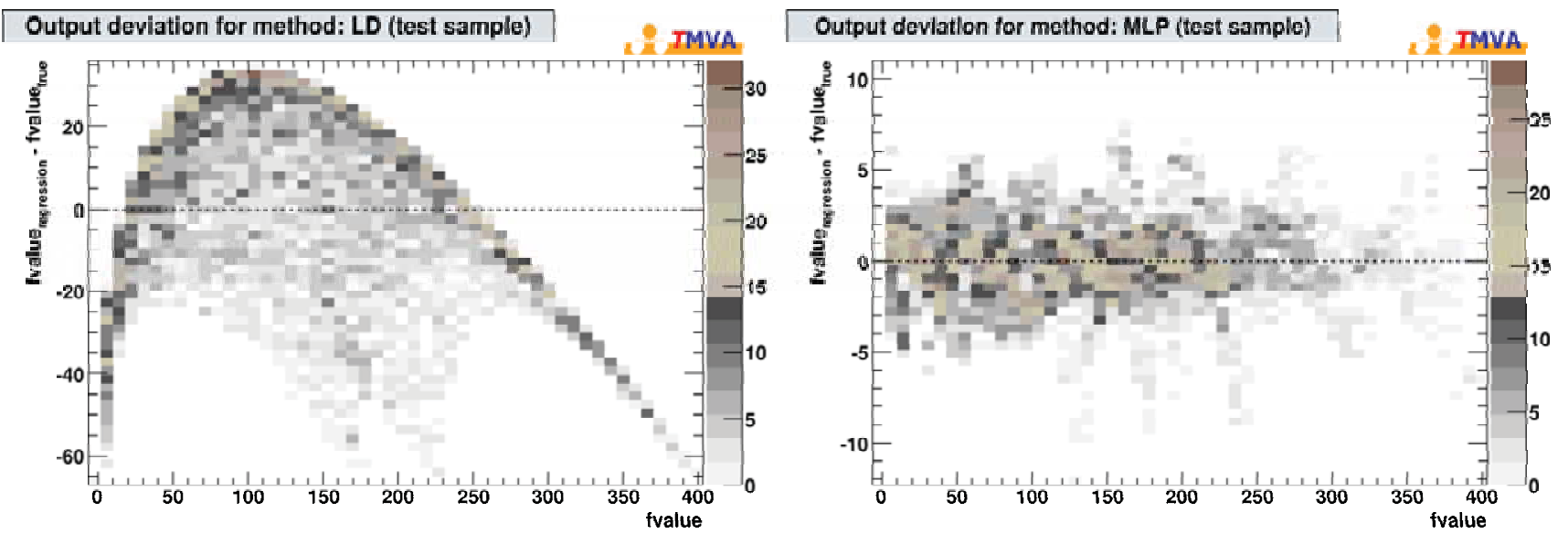

FIG. 8: Example plots for the deviation between regression output and target values for a Linear Discriminant (LD - left) and MLP (right). The dependence of the input variables on the target being strongly nonlinear, LD cannot appropriately solve the regression problem.

world use cases this is not often the case, so that sometimes only little additional information can be recovered by the decorrelation procedure. For highly nonlinear problems the performance may even become worse with linear decorrelation. Nonlinear methods without prior variable decorrelation should be used in such cases.

\subsubsection{Principal component decomposition}

Principal component decomposition or principal component analysis (PCA) as presently applied in TMVA is not very different from the above linear decorrelation. In common words, PCA is a linear transformation that rotates a sample of data points such that the maximum variability is visible. It thus identifies the most important gradients. In the PCA-transformed coordinate system, the largest variance by any projection of the data comes to lie on the first coordinate (denoted the first principal component), the second largest variance on the second coordinate, and so on. PCA can thus be used to reduce the dimensionality of a problem (initially given by the number of input variables) by removing dimensions with insignificant variance. This corresponds to keeping lower-order principal components and ignoring higher-order ones. This latter step however goes beyond straight variable transformation as performed in 
the preprocessing steps discussed here (it rather represents itself a full classification method). Hence all principal components are retained here.

The tuples $\mathbf{x}_{U}^{\mathrm{PC}}(i)=\left(x_{U, 1}^{\mathrm{PC}}(i), \ldots, x_{U, n_{\text {ver }}}^{\mathrm{PC}}(i)\right)$ of principal components of a tuple of input variables $\mathbf{x}(i)=\left(x_{1}(i), \ldots, x_{n_{\text {var }}}(i)\right)$, measured for the event $i$ for signal $(U=S)$ and background $(U=B)$, are obtained by the transformation

$$
x_{U, k}^{\mathrm{PC}}(i)=\sum_{\ell=1}^{n_{\mathrm{var}}}\left(x_{U, \ell}(i)-\bar{x}_{U, \ell}\right) v_{U, \ell}^{(k)}, \quad \forall k=1, n_{\mathrm{var}} .
$$

The tuples $\overline{\mathbf{x}}_{U}$ and $\mathbf{v}_{U}^{(k)}$ are the sample means and eigenvectors, respectively. They are computed by the ROOT class TPrincipal. The matrix of eigenvectors $V_{U}=\left(\mathbf{v}_{U}^{(1)}, \ldots, \mathbf{v}_{U}^{\left(n_{\mathrm{var}}\right)}\right)$ obeys the relation

$$
C_{U} \cdot V_{U}=D_{U} \cdot V_{U},
$$

where $C$ is the covariance matrix of the sample $U$, and $D_{U}$ is the tuple of eigenvalues. As for the preprocessing described in Sec. 3.1.2, the transformation (16) eliminates linear correlations for Gaussian variables.

\subsubsection{Gaussian transformation of variables ("Gaussianisation")}

The decorrelation methods described above require linearly correlated and Gaussian distributed input variables. In real-life HEP applications this is however rarely the case. One may hence transform the variables prior to their decorrelation such that their distributions become Gaussian. The corresponding transformation function is conveniently separated into two steps : first, transform a variable into a uniform distribution using its cumulative distribution function ${ }^{8}$ obtained from the training data (this transformation is identical to the "Rarity" introduced in Sec. 2.1 .5 on page 8); second, use the inverse error function to transform the uniform distribution into the desired Gaussian shape with zero mean and unity width. As for the other transformations, one needs to choose which class of events is to be transformed and hence: for classification (Gaussianisation is not available for regression), it is only possible to transform signal or background into proper Gaussian distributions (except for classifiers testing explicitly both hypotheses such as likelihood methods). Hence a discriminant input variable $x$ with probability density function $\hat{x}$ is transformed as follows

$$
x \mapsto \sqrt{2} \cdot \operatorname{erf}^{-1}\left(2 \cdot \int_{-\infty}^{x} \hat{x}\left(x^{\prime}\right) d x^{\prime}-1\right) .
$$

A subsequent decorrelation of the transformed variable tuple sees Gaussian distributions, but most likely non-linear correlations as a consequence of the transformation (18). The distributions obtained after the decorrelation may thus not be Gaussian anymore. It has been suggested that iterating Gaussianisation and decorrelation more than once may improve the performance of likelihood methods (see next section).

\subsubsection{Booking and chaining transformations}

Variable transformations to be applied prior to the MVA training (and application) can be defined independently for each MVA method using the booking option. The available transformation types normalisation, decorrelation, principal component analysis and Gaussianisation can also be arbitrarily chained and iterated. Like this the Gaussianisation can be applied before a decorrelation is attempted.

\subsection{Binary search trees}

When frequent iterations over the training sample need to be performed, it is helpful to sort the sample before using it. Event sorting in binary trees is employed by the MVA methods rectangular cut

\footnotetext{
${ }^{8}$ The cumulative distribution function $F(x)$ of the variable $x$ is given by the integral $F(x)=\int_{-\infty}^{x} \hat{x}\left(x^{\prime}\right) d x^{\prime}$, where $\hat{x}$ is the probability density function of $x$.
} 
optimisation, PDE-RS and k-NN. While the former two classifiers rely on the simplest possible binary tree implementation, k-NN uses a better performing $k d$-tree (cf. Ref. [14]).

Efficiently searching for and counting events that lie inside a multidimensional volume spanned by the discriminating input variables is accomplished with the use of a binary tree search algorithm [15]. ${ }^{9}$ The amount of computing time needed to sort $N$ events into the tree is $[16] \propto \sum_{i=1}^{N} \ln _{2}(i)=\ln _{2}(N !) \simeq N \ln _{2} N$. Finding the events within the tree which lie in a given volume is done by comparing the bounds of the volume with the coordinates of the events in the tree. Searching the tree once requires a CPU time that is $\propto \ln _{2} N$, compared to $\propto N^{n_{\text {var }}}$ without prior event sorting.

\section{Probability Density Functions}

Several methods and functionalities in TMVA require the estimation of probability densities (PDE) of one or more correlated variables. One may distinguish three conceptually different approaches to PDEs : (i) parametric approximation, where the training data are fitted with a user-defined parametric function. (ii) nonparametric approximation, where the data are fitted piecewise using simple standard functions, such as a polynomial or a Gaussian, and (iii) nearest-neighbour estimation, where the average of the training data in the vicinity of a test event determines the PDF.

All multidimensional PDEs used in TMVA are based on nearest-neighbour estimation with however quite varying implementations. They are described in Secs. 7.3, 7.4 and 7.5 .

One-dimensional PDFs in TMVA are estimated by means of nonparametric approximation, because parametric functions cannot be generalised to a-priori unknown problems. The training data can be in form of binned histograms, or unbinned data points (or "quasi-unbinned" data, i.e., histograms with very narrow bins). In the first case, the bin centres are interpolated with polynomial spline curves, while in the latter case one attributes a kernel function to each event such that the PDF is represented by the sum over all kernels. Beside a faithful representation of the training data, it is important that statistical fluctuations are smoothed out as much as possible without destroying significant information. In practise, where the true PDFs are unknown, a compromise determines which information is regarded significant and which is not. Likelihood methods crucially depend on a good-quality PDF representation. Since the PDFs are strongly problem dependent, the default configuration settings in TMVA will almost never be optimal. The user is therefore advised to scrutinise the agreement between training data and PDFs via the available plotting macros, and to optimise the settings.

\section{$5 \quad$ Optimisation and Fitting}

Several MVA methods (notably cut optimisation and FDA) require general purpose parameter fitting to optimise the value of an estimator. For example, an estimator could be the sum of the deviations of classifier outputs from 1 for signal events and 0 for background events, and the parameters are adjusted so that this sum is as small as possible. Since the various fitting problems call for dedicated solutions, TMVA has a fitter base class, used by the MVA methods, from which all concrete fitters inherit. The consequence of this is that the user can choose whatever fitter is deemed suitable and can configure it through the option string of the MVA method. At present, four fitters are implemented and described below : Monte Carlo sampling, Minuit minimisation, a Genetic Algorithm, Simulated Annealing. They are selected via the configuration option of the corresponding MVA method for which the fitter is invoked.

${ }^{9}$ The following is extracted from Ref. [16] for a two-dimensional range search example. Consider a random sequence of signal events $e_{i}\left(x_{1}, x_{2}\right), i=1,2, \ldots$, which are to be stored in a binary tree. The first event in the sequence becomes by definition the topmost node of the tree. The second event $e_{2}\left(x_{1}, x_{2}\right)$ shall have a larger $x_{1}$ coordinate than the first event, therefore a new node is created for it and the node is attached to the first node as the right child (if the $x_{1}$-coordinate had been smaller, the node would have become the left child). Event $e_{3}$ shall have a larger $x_{1}$-coordinate than event $e_{1}$, it therefore should be attached to the right branch below $e_{1}$. Since $e_{2}$ is already placed at that position, now the $x_{2}$-coordinates of $e_{2}$ and $e_{3}$ are compared, and, since $e_{3}$ has a larger $x_{2}, e_{3}$ becomes the right child of the node with event, $e_{2}$. The tree is sequentially filled by taking every event and, while descending the tree, comparing its $x_{1}$ and $x_{2}$ coordinates with the events already in place. Whether $x_{1}$ or $x_{2}$ are used for the comparison depends on the level within the tree. On the first level, $x_{\text {i }}$ is used, on the second level $x_{2}$, on the third again $x_{1}$ and so on. 


\subsection{Monte Carlo sampling}

The simplest and most straightforward, albeit inefficient fitting method is to randomly sample the fit parameters and to choose the set of parameters that optimises the estimator. The priors used for the sampling are uniform or Gaussian within the parameter limits. The specific behaviour for the MC sampling can be changed using the appropriate configurations options.

Minuit is the standard multivariate minimisation package used in HEP [19]. Its purpose is to find the minimum of a multi-parameter estimator function and to analyse the shape of the function around the minimum (error analysis). The principal application of the TMVA fitters is simple minimisation, while the shape of the minimum is irrelevant in most cases. The use of Minuit is therefore not necessarily the most efficient solution, but because it is a very robust tool we have included it here. Minuit searches the solution along the direction of the gradient until a minimum or an boundary is reached (MIGRAD algorithm). It does not attempt to find the global minimum but is satisfied with local minima. If during the error analysis with MINOS, the minimum smailer values than the local minimum might be obtained. In particular, the use of MINOS may as a side effect of an improved error analysis uncover a convergence in a local minimum, in which case MIGRAD minimisation is invoked once again. If multiple local and/or global solutions exist, it might be preferable to use any of the other fitters which are specifically designed for this type of problem.

\subsection{Genetic Algorithm}

A Genetic Algorithm is a technique to find approximate solutions to optimisation or search problems. The problem is modelled by a group (population) of abstract representations (genomes) of possible solutions (individuals). By applying means similar to processes found in biological evolution the individuals of the population should evolve towards an optimal solution of the problem. Processes which are usually modelled in evolutionary algorithms - of which Genetic Algorithms are a subtype - are inheritance, mutation and "sexual recombination" (also termed crossover).

Apart from the abstract representation of the solution domain, a fitness function must be defined. Its purpose is the evaluation of the goodness of an individual. The fitness function is problem dependent. It either returns a value representing the individual's goodness or it compares two individuals and indicates which of them performs better.

The TMVA Genetic Algorithm provides controls that are as ususal set through configuration options. The user can specify for example the number of individuals created at each generation of the Genetic Algorithm, steer the size and rate of mutations etc. More guidelines of how to choose the best configuration of the Genetic Algorithm and all the options are given in the Users Guide [6].

\subsection{Simulated Annealing}

Simulated Annealing also aims at solving a minimisation problem with several discrete or continuous, local or global minima. The algorithm is inspired by the process of of annealing which occur in condensed matter physics. When first heating and then slowly cooling down a metal ("annealing") its atoms move towards a state of lowest energy, while for sudden cooling the atoms tend to freeze in intermediate states higher energy. For infinitesimal annealing activity the system will always converge in its global energy minimum (see, e.g., Ref. [20]). This physical principle can be converted into an algorithm to achieve slow, but correct convergence of an optimisation problem with multiple solutions. Recovery out of local minima is achieved by assigning the probability [21]

$$
p(\Delta E) \propto \exp \left(-\frac{\Delta E}{T}\right),
$$

to a perturbation of the parameters leading to a shift $\Delta E$ in the energy of the system. The probability of such perturbations to occur decreases with the size of a positive energy coefficient of the perturbation, and increases with the ambient temperature $(T)$.

The TMVA implementation of Simulated Annealing includes various different adaptive adjustments of the perturbation and temperature gradients, that can be used to optimize the performance. 


\subsection{Combined fitters}

For MVA methods such as FDA, where parameters of a discrimination function are adjusted to achieve optimal classification performance (cf. Sec. 7.9), the user can choose to combine Minuit parameter fitting with Monte Carlo sampling or a Genetic Algorithm. While the strength of Minuit is the speedy detection of a nearby local minimum, it might not find a better global minimum. If several local minima exist Minuit will find different solutions depending on the start values for the fit parameters. When combining Minuit with Monte Carlo sampling or a Genetic Algorithm, Minuit uses starting values generated by these methods. The subsequent fits then converge in local minima. Such a combination is usually more efficient than the uncontrolled sampling used in Monte Carlo techniques. When combined with a Genetic Algorithm the user can benefit from the advantages of both methods : the Genetic Algorithm to roughly locate the global minimum, and Minuit to find an accurate solution for it (for an example see the FDA method).

\section{Boosting and Bagging}

Boosting is a way of enhancing the classification and regression performance (and increasing the stability with respect to statistical fluctuations in the training sample) of typically weak MVA methods by sequentially applying an MVA algorithm to reweighted (boosted) versions of the training data and then taking a weighted majority vote of the sequence of MVA algorithms thus produced. It has been introduced to classification techniques in the early ' 90 s [27] and in many cases this simple strategy results in dramatic performance increases.

Although one may argue that bagging (cf. Sec. 6.3) is not a genuine boosting algorithm, we include it in the same context and typically when discussing boosting we also refer to bagging. The most commonly boosted methods are decision trees. However, as described in Sec. 8.1 on page 49, any MVA method may be boosted with TMVA. Hence, although the following discussion refers to decision trees, it also applies to other methods. (Note however that "Gradient Boost" is only available for decision trees and only for classification in the present TMVA version).

\subsection{Adaptive Boost (AdaBoost)}

The most popular boosting algorithm is the so-called AdaBoost (adaptive boost) [28]. In a classification problem, events that were misclassified during the training of a decision tree are given a higher event weight in the training of the following tree. Starting with the original event weights when training the first decision tree, the subsequent tree is trained using a modified event sample where the weights of previously misclassified events are multiplied by a common boost weight $\alpha$. The boost weight is derived from the misclassification rate, err, of the previous tree ${ }^{10}$.

$$
\alpha=\frac{1-\text { err }}{\text { err }} .
$$

The weights of the entire event sample are then renormalised such that the sum of weights remains constant.

We define the result of an individual classifier as $h(\mathbf{x})$, with ( $\mathbf{x}$ being the tuple of input variables) encoded for signal and background as $h(\mathbf{x})=+1$ and -1 , respectively. The boosted event classification $y_{\text {Boost }}(\mathrm{x})$ is then given by

$$
y_{\text {Boost }}(\mathbf{x})=\frac{1}{N_{\text {collection }}} \cdot \sum_{i}^{N_{\text {collection }}} \ln \left(\alpha_{i}\right) \cdot h_{i}(\mathbf{x}),
$$

where the sum is over all classifiers in the collection. Small (large) values for $y_{\text {Boost }}(\mathbf{x})$ indicate a backgroundlike (signal-like) event. Equation (21) represents the default boosting algorithm. It can be modified via

\footnotetext{
${ }^{10}$ By construction, the error rate is err $\leq 0.5$ as the same training events used to classify the output nodes of the previous tree are used for the calculation of the error rate.
} 
the configuration option string of the MVA method to be boosted, e.g. if one if one wants to use an unweighted average of the boosted decision trees or classifiers instead of the weighted one.

For regression trees, the AdaBoost algorithm needs to be modified. TMVA uses here the so-called AdaBoost.R2 algorithm [29]. The idea is similar to AdaBoost albeit with a redefined loss per event to account for the the deviation of the estimated target value from the true one. Moreover, as there are no longer correctly and wrongly classified events, all events need to be reweighted depending on their individual loss, which - for event $k$ - is given by

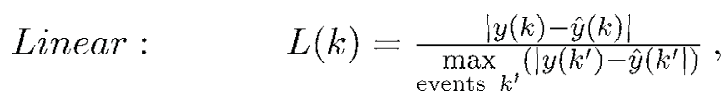

$$
\begin{aligned}
& \text { Square: } \quad L(k)=\left[\frac{|y(k)-\hat{y}(k)|}{\max _{\text {events } k^{\prime}}\left(\mid y\left(k^{\prime}\right)-\hat{y}\left(k^{\prime}\right)\right)}\right]^{2} \text {, } \\
& \text { Exponential : } \quad L(k)=1-\exp \left[-\frac{|y(k)-\hat{y}(k)|}{\max _{\text {events } k^{\prime}}\left(\mid y\left(k^{\prime}\right)-\hat{y}^{\prime}\left(k^{\prime}\right)\right.}\right] .
\end{aligned}
$$

The average loss of the classifier $y^{(i)}$ over the whole training sample, $\langle L\rangle^{(i)}=\sum_{\text {events } k^{\prime}} w\left(k^{\prime}\right) L^{\langle i\rangle}\left(k^{\prime}\right\rangle$. can be considered to be the analogon to the error fraction in classification. Given $\langle L\rangle$, one computes the quantity $\beta_{(i)}=\langle L\rangle^{(i)} /\left(1-\langle L\rangle^{(i)}\right)$, which is used in the boosting of the events, and for the combination of the regression methods belonging to the boosted collection. The boosting weight, $w^{(i+1)}(k)$, for event $k$ and boost step $i+1$ thus reads

$$
w^{(i+1)}(k)=w^{(i)}(k) \cdot \beta_{(i)}^{1-L^{(i)}(k)} .
$$

The sum of the event weights is again renormalised to reproduce the original overall number of events. The final regressor, $y_{\text {Boost }}$, uses the weighted median, $\tilde{y}_{(i)}$, where $(i)$ is chosen so that it is the minimal (i) that satisfies the inequality

$$
\sum_{\substack{t \in \text { sorted collection } \\ t \leq \imath}} \ln \frac{1}{\beta_{(t)}} \geq \frac{1}{2} \sum_{t}^{N_{\text {collection }}} \ln \frac{1}{\beta_{(t)}}
$$

\subsection{Gradient Boost}

The idea of function estimation through boosting can be understood by considering a simple additive expansion approach. The function $F(\mathbf{x})$ under consideration is assumed to be a weighted sum of parametrised base functions $f\left(x ; a_{m}\right)$, so-called "weak learners". From a technical point of view any TMVA classifier could act as a weak learner in this approach, but decision trees benefit most from boosting and are currently the only classifier that implements GradientBoost (a generalisation may be included in future releases). Thus each base function in this expansion corresponds to a decision tree

$$
F(\mathbf{x} ; P)=\sum_{m=0}^{M} \beta_{m} f\left(x ; a_{m}\right) ; \quad P \in\left\{\beta_{m} ; a_{m}\right\}_{0}^{M} .
$$

The boosting procedure is now employed to adjust the parameters $P$ such that the deviation between the model response $F(\mathrm{x})$ and the true value $y$ obtained from the training sample is minimised. The deviation is measured by the so-called loss-function $L(F, y)$, a popular choice being squared error loss $L(F, y)=(F(\mathrm{x})-y)^{2}$. It can be shown that the loss function fully determines the boosting procedure.

The most popular boosting method, AdaBoost, is based on exponential loss, $L(F, y)=e^{-F(\mathbf{x}) y}$, which leads to the well known reweighting algorithm described in Sec. 6.1. Exponential loss has the shortcoming that it lacks robustness in presence of outliers or mislabelled data points. The performance of AdaBoost therefore degrades in noisy settings.

The GradientBoost algorithm attempts to cure this weakness by allowing for other, potentially more robust, loss functions without giving up on the good out-of-the-box performance of AdaBoost. The current 
TMVA implementation of GradientBoost uses the binomial log-likelihood loss

$$
L(F, y)=\ln \left(1+e^{-2 F(\mathbf{x}) y}\right)
$$

for classification. As the boosting algorithm corresponding to this loss function cannot be obtained in a straightforward manner, one has to resort to a steepest-descent approach to do the minimisation. This is done by calculating the current gradient of the loss function and then growing a regression tree whose leaf values are adjusted to match the mean value of the gradient in each region defined by the tree structure. Iterating this procedure yields the desired set of decision trees which minimises the loss function. Note that GradientBoost can be adapted to any loss function as long as the calculation of the gradient is feasible.

Giving good results already for small trees (5-10 leaf nodes), GradientBoost is typically less susceptible to overtraining. Its robustness can be enhanced by reducing the learning rate of the algorithm through the Shrinkage parameter, which controls the weight of the individual trees. A small shrinkage (0.1-0.3) demands more trees to be grown but can significantly improve the accuracy of the prediction in difficult settings.

In certain settings GradientBoost may also benefit from the introduction of a bagging-like resampling procedure using random subsamples of the training events for growing the trees. This is called stochastic gradient boosting and can be enabled by selecting the UseBaggedGrad option. The sample fraction used in each iteration can be controlled through the parameter GradBaggingFraction, where typically the best results are obtained for values between 0.5 and 0.8 .

For regression tasks, GradientBoost employs the Huber loss function [47], which features the robustness of least-absolute-deviation loss for error distributions with broad tails, while maintaining the high efficiency of least-squares loss for normally distributed errors. For moderately broad tails, it should surpass both least-squares and least-absolute-deviation loss.

$$
L(F, y)=\left\{\begin{array}{cc}
\frac{1}{2}(y-F(\mathbf{x}))^{2} & |y-F| \leq \delta, \\
\delta(|y-F|-\delta / 2) & |y-F|>\delta .
\end{array}\right.
$$

All GradientBoost options for classification described above can be also applied for regression tasks, but tests have shown that stochastic gradient boosting may not perform very well for regression problems.

\subsection{Bagging}

The term Bagging denotes a resampling technique where a classifier is repeatedly trained using resampled training events such that the combined classifier represents an average of the individual classifiers. A priori, bagging does not aim at enhancing a weak classifier in the way adaptive or gradient boosting does, and is thus not a "boosting" algorithm in a strict sense. Instead it effectively smears over statistical representations of the training data and is hence suited to stabilise the response of a classifier. In this context it is often accompanied also by a significant performance increase compared to the individual classifier.

Resampling includes the possibility of replacement, which means that the same event is allowed to be (randomly) picked several times from the parent sample. This is equivalent to regarding the training sample as being a representation of the probability density distribution of the parent sample : indeed, if one draws an event out of the parent sample, it is more likely to draw an event from a region of phase-space that has a high probability density, as the original data sample will have more events in that region. If a selected event is kept in the original sample (that is when the same event can be selected several times), the parent sample remains unchanged so that the randomly extracted samples will have the same parent distribution, albeit statistically fluctuated. Training several classifiers with different resampled training data, and combining them into a collection, results in an averaged classifier that, just as for boosting, is more stable with respect to statistical fluctuations in the training sample.

Technically, resampling is implemented by applying random Poisson weights to each event of the parent sample. 


\section{The TMVA Methods}

All TMVA classification and regression methods (in most cases, a method serves both analysis goals) inherit from a common base class, which implements functionality like the interpretation of common configuration options, the interaction with the training and test data sets, $1 / O$ operations and common performance evaluation calculus. Each classifier output can be fitted by a PDF to allow the computation of the Rarity, or to input it to subsequent likelihood fits. The following sections describe the methods implemented in TMVA.

\subsection{Rectangular cut optimisation}

The simplest and most common classifier for selecting signal events from a mixed sample of signal and background events is the application of an ensemble of rectangular cuts on discriminating variables. Unlike all other classifiers in TMVA, the cut classifier only returns a binary response (signal or background). ${ }^{11}$ The optimisation of cuts performed by TMVA maximises the background rejection at given signal efficiency, and scans over the full range of the latter quantity. Dedicated analysis optimisation for which, e.g., the signal significance is maximised requires the expected signal and background yields to be known before applying the cuts. This is not the case for a multi-purpose discrimination and hence not used by TMVA. However, the cut ensemble leading to maximum significance corresponds to a particular working point on the efficiency curve, and can hence be easily derived after the cut optimisation scan has converged. ${ }^{12}$

TMVA cut optimisation is performed with the use of multivariate parameter fitters for which only Monte Carlo sampling, Genetic Algorithm, and Simulated Annealing showed satisfying results (gradientbased fitting (Minuit) is unsuccessful due to the non-unique solution space). The training events are sorted in binary trees prior to the optimisation, which significantly reduces the computing time required to determine the number of events passing a given cut ensemble (cf. Sec. 3.2).

The cut optimisation analysis proceeds by first building binary search trees for signal and background. For each variable, statistical properties like mean, root-mean-squared (RMS), variable ranges are computed to guide the search for optimal cuts. Cut optimisation requires an estimator that quantifies the goodness of a given cut ensemble. Maximising this estimator minimises (maximises) the background efficiency, $\varepsilon_{B}$ (background rejection, $r_{B}=1-\varepsilon_{B}$ ) for each signal efficiency $\varepsilon_{S}$.

All optimisation methods (fitters) act on the assumption that one minimum and one maximum requirement on each variable is sufficient to optimally discriminate signal from background (i.e., the signal is clustered). If this is not the case, the variables must be transformed prior to the cut optimisation to make them compliant with this assumption.

For a given cut ensemble the signal and background efficiencies are derived by counting the training events that pass the cuts and dividing the numbers found by the original sample sizes. The resulting efficiencies are therefore rational numbers that may exhibit visible discontinuities when the number of training events is small and an efficiency is either very small or very large. Another way to compute efficiencies is to parameterise the probability density functions of all input variables and thus to achieve continuous efficiencies for any cut vahe. Note however that this method expects the input variables to be uncorrelated! Non-vanishing correlations would lead to incorrect efficiency estimates and hence to underperforming cuts.

\footnotetext{
11 Note that cut optimisation is not a multivariate analyser method but a sequence of univariate ones, because no combination of the variables is achieved. Neither does a cut on one variable depend on the value of another variable (like it is the case for Decision Trees), nor can a, say, background-like value of one variable in a signal event be counterweighed by signal-like values of the other variables (like it is the case for the likelihood method).

${ }^{12}$ Assuming a large enough number of events so that Gaussian statistics is applicable, the significance for a signal is given by $\mathcal{S}=\varepsilon_{S} \mathcal{N}_{S} / \sqrt{\varepsilon_{\mathcal{S}} \mathcal{N}_{S}+\varepsilon_{\mathcal{B}}\left(\varepsilon_{S}\right) \mathcal{N}_{S}}$, where $\varepsilon_{S(B)}$ and $N_{S(B)}$ are the signal and background efficiencies for a cut ensemble and the event yields before applying the cuts, respectively. The background efficiency $\varepsilon_{B}$ is expressed as a function of $\varepsilon_{S}$ using the TMVA evaluation curve obtained form the test data sample. The maximum significance is then found at the root of the derivative
}

which depends on the problem.

$$
\frac{d \mathcal{S}}{d \varepsilon_{S}}=N_{S} \frac{2 \varepsilon_{B}\left(\varepsilon_{S}\right) N_{B}+\varepsilon_{S}\left(N_{S}-\frac{d \varepsilon_{B}\left(\varepsilon_{S}\right)}{d \varepsilon_{S}} N_{B}\right)}{2\left(\varepsilon_{S} N_{S}+\varepsilon_{B}\left(\varepsilon_{S}\right) N_{B}\right)^{3 / 2}}=0
$$




\section{Monte Carlo sampling}

Each generated cut sample (cf. Sec. 5.1) corresponds to a point in the $\left(\varepsilon_{S}, r_{B}\right)$ plane. The $\varepsilon_{S}$ dimension is (finely) binned and a cut sample is retained if its $r_{B}$ value is larger than the value already contained in that bin. This way a reasonably smooth efficiency curve can be obtained if the number of input variables is not too large (the required number of $\mathrm{MC}$ samples grows with powers of $2 n_{\mathrm{var}}$ ).

Prior information on the variable distributions can be used to reduce the number of cuts that need to be sampled. For example, if a discriminating variable follows Gaussian distributions for signal and background, with equal width but a larger mean value for the background distribution, there is no useful minimum requirement (other than $-\infty$ ) so that a single maximum requirement is sufficient for this variable. To instruct TMVA to remove obsolete requirements, the option VarProp [i] must be used, where [i] indicates the counter of the variable (following the order in which they have been registered with the Factory, beginning with 0) must be set to either FMax or FMin. TMVA is capable of automatically detecting which of the requirements should be removed. Use the option VarProp [i]=FSmart (where again [i] must be replaced by the appropriate variable counter, beginning with 0 ). Note that in many realistic use cases the mean values between signal and background of a variable are indeed distinct, but the background can have large tails. In such a case, the removal of a requirement is inappropriate, and would lead to underperforming cuts.

\section{Genetic Algorithm}

Genetic Algorithm (cf. Sec. 5.2) is a technique to find approximate solutions to optimisation or search problems. Apart from the abstract representation of the solution domain, a fitness function must be defined. In cut optimisation, the fitness of a rectangular cut is given by good background rejection combined with high signal efficiency.

At the initialization step, all parameters of all individuals (cut ensembles) are chosen randomly. The individuals are evaluated in terms of their background rejection and signal efficiency. Each cut ensemble giving an improvement in the background rejection for a specific signal efficiency bin is immediately stored. Each individual's fitness is assessed, where the fitness is largely determined by the difference of the best found background rejection for a particular bin of signal efficiency and the value produced by the current individual. The same individual that has at one generation a very good fitness will have only average fitness at the following generation. This forces the algorithm to focus on the region where the potential of improvement is the highest. Individuals with a good fitness are selected to produce the next generation. The new individuals are created by crossover and mutated afterwards. Mutation changes some values of some parameters of some individuals randomly following a Gaussian distribution function, etc.

\section{Simulated Annealing}

Cut optimisation using Simulated Annealing works similarly as for the Genetic Algorithm and achieves comparable performance. The same fitness function is used to estimator the goodness of a given cut ensemble. Details on the algorithm and the configuration options can be found in Sec. 5.3 on page 18 .

\section{Performance}

The Genetic Algorithm currently provides the best cut optimisation convergence. However, it is found that with rising number of discriminating input variables the goodness of the solution found (and hence the smoothness of the background-rejections versus signal efficiency plot) deteriorates quickly. Rectangular cut optimisation should therefore be reduced to the variables that have the largest discriminating power.

If variables with excellent signal from background separation exist, applying cuts can be quite competitive with more involved classifiers. Cuts are known to underperform in presence of strong nonlinear correlations and/or if several weakly discriminating variables are used. In the latter case, a true multivariate combination of the information will be rewarding. 


\subsection{Projective likelihood estimator (PDE approach)}

The method of maximum likelihood consists of building a model out of probability density functions (PDF) that reproduces the input variables for signal and background. For a given event, the likelihood for being of signal type is obtained by multiplying the signal probability densities of all input variables, which are assumed to be independent, and normalising this by the sum of the signal and background likelihoods. Because correlations among the variables are ignored, this PDE approach is also called "naive Bayes estimator", unlike the full multidimensional PDE approaches such as PDE-range search, PDE-foam and $k$-nearest-neighbour discussed in the subsequent sections, which approximate the true Bayes limit.

The likelihood ratio $y_{\mathcal{L}}(i)$ for event $i$ is defined by

$$
y_{\mathcal{L}}(i)=\frac{\mathcal{L}_{S}(i)}{\mathcal{L}_{S}(i)+\mathcal{L}_{B}(i)}
$$

where

$$
\mathcal{L}_{S(B)}(i)=\prod_{k=1}^{n_{\text {var }}} p_{S(B), k}\left(x_{k}(i)\right),
$$

and where $p_{S(B), k}$ is the signal (background) PDF for the $k$ th input variable $x_{k}$. The PDFs are normalised

$$
\int_{-\infty}^{+\infty} p_{S(B), k}\left(x_{k}\right) d x_{k}=1, \quad \forall k .
$$

It can be shown that in absence of model inaccuracies (such as correlations between input variables not removed by the de-correlation procedure, or an inaccurate probability density model), the ratio (31) provides optimal signal from background separation for the given set of input variables.

Since the parametric form of the PDFs is generally unknown, the PDF shapes are empirically approximated from the training data by nonparametric functions, which can be chosen individually for each variable and are either polynomial splines of various degrees fitted to histograms or unbinned kernel density estimators (KDE), as discussed in Sec. (4).

A certain number of primary validations are performed during the PDF creation, the results of which are printed to standard output. Among these are the computation of a $\chi^{2}$ estimator between all nonzero bins of the original histogram and its PDF, and a comparison of the number of outliers (in sigmas) found in the original histogram with respect to the (smoothed) PDF shape, with the statistically expected one. The fidelity of the PDF estimate can be also inspected visually by executing the macro likelinoodrefs.C (cf. Table 4).

\section{Transforming the likelihood output}

If a data-mining problem offers a large number of input variables, or variables with excellent separation power, the likelihood response $y_{\mathcal{L}}$ is often strongly peaked at 0 (background) and 1 (signal). Such a response is inconvenient for the use in subsequent analysis steps. TMVA therefore allows to transform the likelihood output by an inverse sigmoid function that zooms into the peaks

$$
y_{\mathcal{L}}(i) \longrightarrow y_{\mathcal{L}}^{\prime}(i)=-\tau^{-1} \ln \left(y_{\mathcal{L}}^{-1}-1\right)
$$

where $\tau=15$ is used. Note that $y_{\mathcal{L}}^{\prime}(i)$ is no longer contained within $[0,1]$ (see Fig. 9).

\section{Performance}

Both the training and the application of the likelihood classifier are very fast operations that are suitable for large data sets.

The performance of the classifier relies on the accuracy of the likelihood model. Because high fidelity PDF estimates are mandatory, sufficient training statistics is required to populate the tails of the distributions. The neglect of correlations between input variables in the model (32), often leads to a diminution 


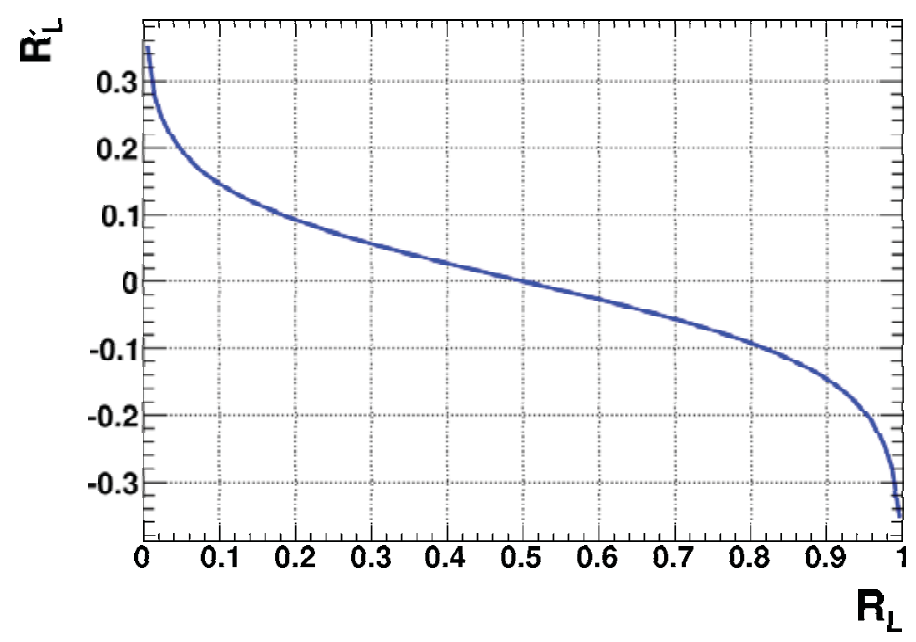

Fig. 9: Transformation (34) of the likelihood output.

of the discrimination performance. While linear Gaussian correlations can be rotated away (see Sec. 3.1), such an ideal situation is rarely given. Positive correlations lead to peaks at both $y_{\mathcal{L}} \rightarrow 0,1$. Correlations can be reduced by categorising the data samples and building an independent likelihood classifier for each event category. Such categories could be geometrical regions in the detector, kinematic properties, etc. In spite of this, realistic applications with a large number of input variables are often plagued by irreducible correlations, so that projective likelihood approaches like the one discussed here are under-performing. This finding led to the development of the many alternative classifiers that exist in statistical theory today.

\subsection{Multidimensional likelihood estimator (PDE range-search approach)}

This is a generalization of the projective likelihood classifier described in Sec. 7.2 to $n_{\text {var }}$ dimensions, where $n_{\text {var }}$ is the number of input variables used. If the multidimensional PDF for signal and background (or regression data) were known, this classifier would exploit the full information contained in the input variables, and would hence be optimal. In practice however, huge training samples are necessary to sufficiently populate the multidimensional phase space. ${ }^{13}$ Kernel estimation methods may be used to approximate the shape of the PDF for finite training statistics.

A simple probability density estimator denoted $P D E$ range search, or $P D E-R S$, has been suggested in Ref. [16]. The PDE for a given test event (discriminant) is obtained by counting the (normalised) number of training events that occur in the "vicinity" of the test event. The classification of the test event may then be conducted on the basis of the majority of the nearest training events. The $n_{\text {var }}$-dimensional volume that encloses the "vicinity" is user-defined and can be adaptive. A search method based on sorted binary trees is used to reduce the computing time for the range search. To enhance the sensitivity within the volume, kernel functions are used to weight the reference events according to their distance from the test event. PDE-RS is a variant of the k-nearest neighbour classifier described in Sec. 7.5.

\section{Classification}

To classify an event as being either of signal or of background type, a local estimate of the probability density of it belonging to either class is computed. The method of PDE-RS provides such an estimate by defining a volume $(V)$ around the test event $(i)$, and by counting the number of signal $\left(n_{S}(i, V)\right)$ and background events $\left(n_{B}(i, V)\right)$ obtained from the training sample in that volume. The ratio

$$
y_{\mathrm{PDE}-\mathrm{RS}}(i, V)=\frac{1}{1+r(i, V)},
$$

\footnotetext{
${ }^{13}$ Due to correlations between the input variables, only a sub-space of the full phase space may be populated.
} 


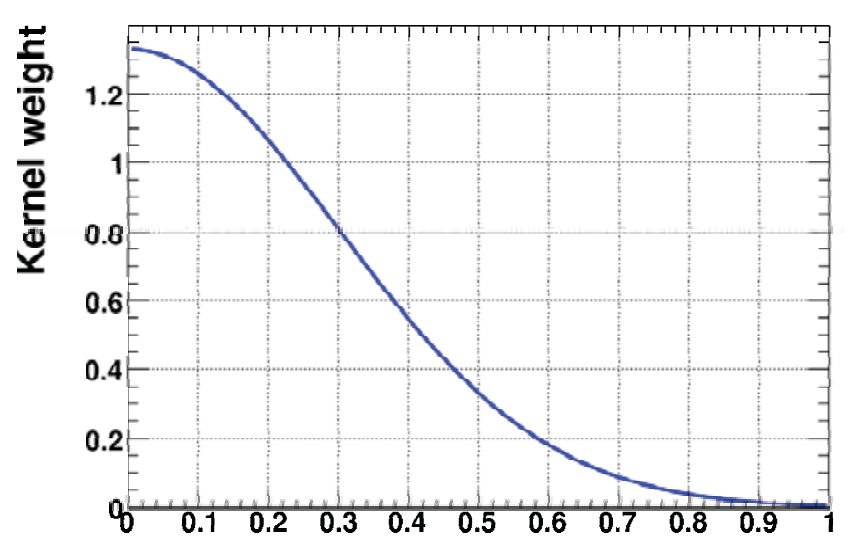

Radial distance from test discriminant

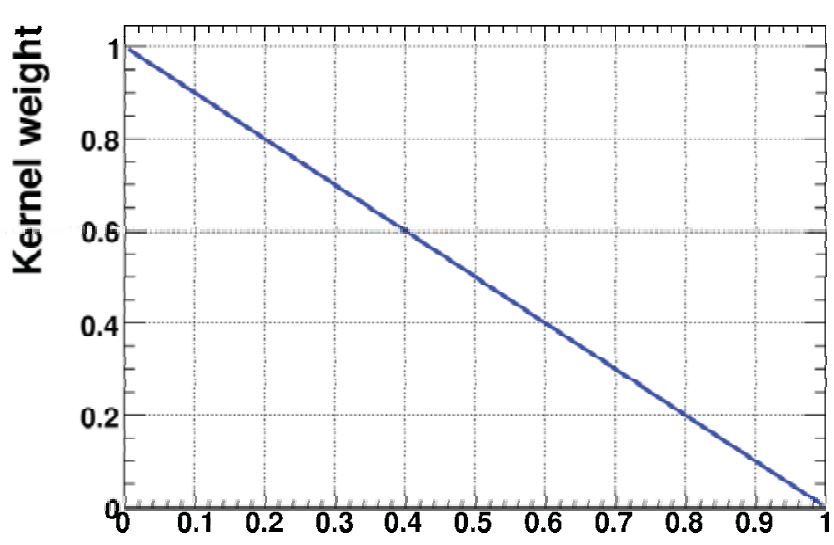

Radial distance from test discriminant

FIG. 10: Kernel functions (left : Gaussian, right : Teepee) used to weight the events that are found inside the reference volume of a test event.

is taken as the estimate, where $r(i, V)=\left(n_{B}(i, V) / N_{B}\right) \cdot\left(N_{S} / n_{S}(i, V)\right)$, and $N_{S(B)}$ is the total number of signal (background) events in the training sample. The estimator $y_{\text {PDE-RS }}(i, V)$ peaks at $1(0)$ for signal (background) events. The counting method averages over the PDF within $V$, and hence ignores the available shape information inside (and outside) that volume.

Efficiently searching for and counting the events that lie inside the volume is accomplished with the use of a $n_{\mathrm{var}}$-variable binary tree search algorithm [15] (cf. Sec. 3.2).

The TMVA implementation of PDE-RS optionally provides four different volume definitions. The simplest volume definition consisting of a rigid box of some size in units of the variables. This method was the one originally used by the developers of PDE-RS [16]. The MinMax volume is a rectangular box defined in each dimension (i.e., input variable) with respect to a fraction of the full range of values found for that dimension in the training sample. The $R M S$ volume uses a fraction of the RMS in each dimension instead of the whole range, thus being more robust against outliers. Finally, the best volume definition is adaptive, where a volume is defined in each dimension with respect to the RMS of that dimension, estimated from the training sample. The overall scale of the volume is adjusted individually for each test event such that the total number of events confined in the volume lies within a user-defined range. The adjustment is performed by a root finder. The requirement to collect a certain number of events in the volume automatically leads to small volume sizes in strongly populated phase space regions, and enlarged volumes in areas where the population is scarce.

Although the adaptive volume adjustment is more flexible and should perform better, it significantly increases the computing time of the PDE-RS discriminant.

One of the shortcomings of the original PDE-RS implementation is its sensitivity to the exact location of the sampling volume boundaries : an infinitesimal change in the boundary placement can include or exclude a training event, thus changing $r(i, V)$ by a finite amount. ${ }^{14}$ In addition, the shape information within the volume is ignored.

The use of kernel functions mitigates these problems by weighting each event within the volume as a function of its distance to the test event. The farer it is away, the smaller is its weight. Among the kernel functions available in TMVA are a sphere, a hyper-elliptic volume element replacing the rectangular box, but not applying event weights, a teepee function linearly weighting events as a function of the distance from the test event, and a Gauss function, which is the simplest well-behaved convolution kernel. Other kernels implemented for test purposes are "Sinc" and "Lanczos" functions $\alpha \sin x / x$ of different (symmetric) orders. They exhibit strong peaks at zero and oscillating tails. The Gaussian and Teepee kernel functions are shown in Fig. 10.

\footnotetext{
${ }^{14}$ Such an introduction of artefacts by having sharp boundaries in the sampled space is an example of Gibbs's phenomenon, and is commonly referred to as ringing or aliasing.
} 


\section{Regression}

Regression with PDE-RS proceeds similar to classification. The difference lies in the replacement of Eq. (35) by the average target value of all events belonging to the volume $V$ defined by event $i$ (the test event)

$$
y_{\mathrm{PDE}-\mathrm{RS}}(i, V)=\langle t(i, V)\rangle=\frac{\sum_{j \in V} w_{j} t_{j} f(\operatorname{dis}(i, j))}{\sum_{j \in V} w_{j} f(\operatorname{dis}(i, j))},
$$

where the sum is over all training events in $V, w_{j}$ and $t_{j}$ are the weight and target value of event $j$ in $V$, $\operatorname{dis}(i, j)$ is a measure of the distance between events $i$ and $j$, and $f(\ldots)$ is a kernel function.

\section{Performance}

As opposed to many of the more sophisticated data-mining approaches, which tend to present the user with a "black box", PDE-RS is simple enough that the algorithm can be easily traced and tuned by hand. PDE-RS can yield competitive performance if the number of input variables is not too large and the statistics of the training sample is ample. In particular, it naturally deals with complex nonlinear variable correlations, the reproduction of which may, for example, require involved neural network architectures.

PDE-RS is a slowly responding classifier. Only the training, i.e., the fabrication of the binary tree is fast, which is usually not the critical part. The necessity to store the entire binary tree in memory to avoid accessing virtual memory limits the number of training events that can effectively be used to model the multidimensional PDF. This is not the case for the other classifiers implemented in TMVA (with some exception for Boosted Decision Trees).

\subsection{Likelihood estimator using self-adapting phase-space binning (PDE-Foam)}

The PDE-Foam method [18] is an extension of PDE-RS, which divides the multi-dimensional phase space in a finite number of hyper-rectangles (cells) of constant event density. This "foam" of cells is filled with averaged probability density information sampled from the training data. For a given number of cells, the binning algorithm adjusts the size and position of the cells inside the multi-dimensional phase space based on a binary split algorithm that minimises the variance of the event density in the cell. The binned event density information of the final foam is stored in cells, organised in a binary tree, to allow a fast and memory-efficient storage and retrieval of the event density information necessary for classification or regression. The implementation of PDE-Foam is based on the Monte-Carlo integration package TFoam [17] included in ROOT.

In classification mode PDE-Foam forms bins of similar density of signal and background events or the ratio of signal to background. In regression mode the algorithm determines cells with small varying regression targets. In the following, we use the term density $(\rho)$ for the event density in case of classification or for the target variable density in case of regression.

Foams for an arbitrary event sample are formed as follows.

1. Setup of binary search trees. A binary search tree is created and filled with the $d$-dimensional event tuples form the training sample as for the PDE-RS method (cf.Sec. 7.3).

2. Initialisation phase. A foam is created, which at first consists of one $d$-dimensional hyper-rectangle (base cell). The coordinate system of the foam is normalised such that the base cell extends from 0 to 1 in each dimension. The coordinates of the events in the corresponding training tree are linearly transformed into the coordinate system of the foam.

3. Growing phase. A binary splitting algorithm iteratively splits cells of the foam along axis-parallel hyperplanes until the maximum number of active cells is reached. The splitting algorithm minimises the relative variance of the density $\sigma_{\rho} /\langle\rho\rangle$ across each cell (cf.Ref. [17]). For each cell random points uniformly distributed over the cell volume are generated. For each of these points a small box centred around this point is defined. The box has a user-defined size in units of the size of the base cell in each dimension. The density is estimated as the number of events contained in this box divided by the volume of the box. ${ }^{15}$ The densities obtained for all sampled points in the cell are

\footnotetext{
${ }^{15}$ In case of regression this is the average target value computed according to Eq. (36), page 27 .
} 


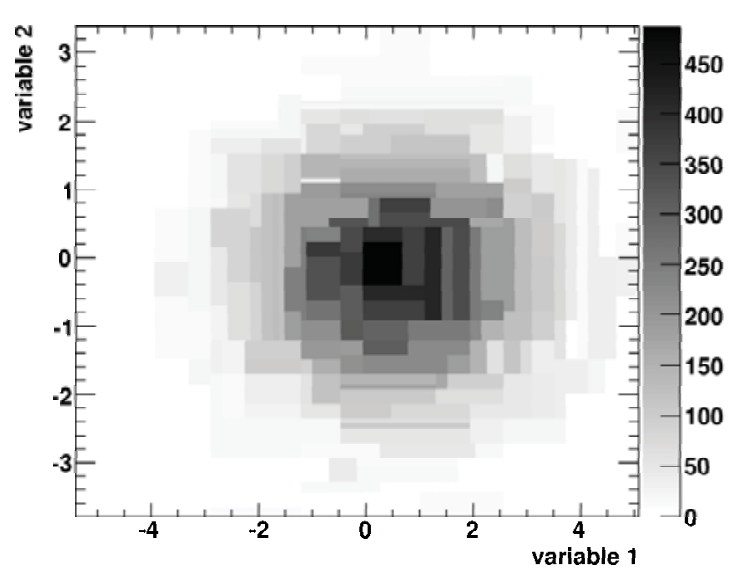

(a) foam projection without kernel

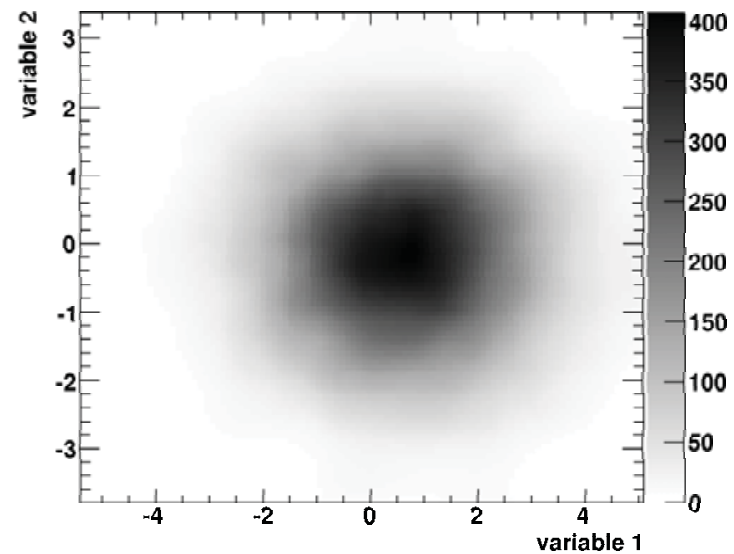

(b) foam projection with Gaussian kernel

FIG. 11: Projections of a two-dimensional foam with 500 cells for a Gaussian distribution on a two-dimensional histogram. The foam was created with 5000 events from the input tree. (a) shows the reconstructed distribution without kernel weighting and (b) shows the distribution weighted with a Gaussian kernel. The grey shades indicate the event density of the drawn cell.

projected onto the $d$ axes of the cell and the projected values are filled in histograms. The next cell to be split and the corresponding division edge (bin) for the split are selected as the ones that have the largest relative variance. The two new daughter cells are marked as 'active' cells and the old mother cell is marked as 'inactive'. A detailed description of the splitting algorithm can be found in Ref. [17]. The geometry of the final foam reflects the distribution of the training samples : phase-space regions where the density is constant are combined in large cells, while in regions with large density gradients many small cells are created. Figure 11(a) displays a foam obtained from a two-dimensional Gaussian-distributed training sample.

4. Filling phase. Each active cell is filled with values that classify the event distribution within this cell and which permit the calculation of the classification or regression discriminator.

5. Evaluation phase. The estimator for a given event is evaluated based on the information stored in the foam cells. The corresponding foam cell, in which the event variables ( $d$-dimensional vector) of a given event is contained, is determined with a binary search algorithm. ${ }^{16}$

The initial trees which contain the training events and which are needed to evaluate the densities for the foam build-up, are discarded after the training phase. The memory consumption for the foam is 160 bytes per foam cell plus an overhead of 1.4 kbytes for the PDE-Foam object on a 64 bit architecture. Note that in the foam all cells created during the growing phase are stored within a binary tree structure. Cells which have been split are marked as inactive and remain empty. To reduce memory consumption, the cell geometry is not stored with the cell, but rather obtained recursively from the information about the division edge of the corresponding mother cell. This way only two short integer numbers per cell represent the information of the entire foam geometry : the division coordinate and the bin number of the division edge.

It is possible to apply a Gaussian kernel smoothing during the evaluation phase. In this case all cells contribute to the calculation of the discriminant for a given event, weighted with their Gaussian distance to the event. The Gaussian kernel avoids discontinuities of the discriminant values at the cell boundaries. In most cases it results in an improved separation power between signal and background. However, the time needed for classification increases due to the larger number of computations performed. A comparison between foams with and without Gaussian kernel can be seen in Fig. 11.

An optional linear interpolation with adjacent cells in each dimension is applied during the classification phase. This results in faster classification than the Gauss weighting of all cells in the foam.

\footnotetext{
${ }^{16}$ For an event that fails outside the foam boundaries, the cell with the smallest Cartesian distance to the event is chosen.
} 
To classify an event in a $d$-dimensional phase space as being either of signal or of background type, a local estimator of the probability that this event belongs to either class can be obtained from the foam's hyper-rectangular cells. The foams are created and filled based on samples of signal and background training events. For classification two possibilities are implemented. One foam can be used to separate the $S / B$ probability density or two separate foams are created, one for the signal events and one for background events. For the same total number of foam cells, the performance of the two implementations is found to be similar.

Two different methods are also implemented for regression. In the first method, applicable for single targets only (mono-target regression), the target value is stored in each cell of the foam. In the second method, applicable to any number of targets (multi-target regression), the target values are stored in higher foam dimensions. In mono-target regression the density used to form the foam is given by the mean target density in a given box. For multi-target regression the target information is stored in additional foam dimensions. For a training sample with $n_{\text {var }}\left(n_{\text {tar }}\right)$ input variables (regression targets), one would form a $\left(n_{\mathrm{var}}+n_{\text {tar }}\right)$-dimensional foam. To compute a target estimate for event $i$, one needs the coordinates of the cell centre $C(i, k)$ in each foam dimension $k$. The kernel weighting methods have been implemented also for regression, taking into account the modified structure of the foam in case of multi-target regression.

\section{Performance}

Like PDE-RS (see Sec. 7.3), this method is a powerful classification tool for problems with highly nonlinearly correlated observables. Furthermore PDE-Foam is a fast responding classifier, because of its limited number of cells, independent of the size of the training samples.

An exception is the multi-target regression with Gauss kernel because the time scales with the number of cells squared. Also the training can be slow, depending on the number of training events and number of cells one wishes to create.

\section{5 k-Nearest Neighbour (k-NN) Classifier}

Similar to PDE-RS (cf. Sec. 7.3), the k-nearest neighbour method compares an observed (test) event to reference events from a training data set [3]. However, unlike PDE-RS, which in its original form uses a fixed-sized multidimensional volume surrounding the test event, and in its augmented form resizes the volume as a function of the local data density, the $\mathrm{k}-\mathrm{NN}$ algorithm is intrinsically adaptive. It searches for a fixed number of adjacent events, which then define a volume for the metric used. The k-NN classifier has best performance when the boundary that separates signal and background events has irregular features that cannot be easily approximated by parametric learning methods.

The k-NN algorithm searches for $k$ events that are closest to the test event. Closeness is thereby measured using a metric function. The simplest metric choice is the Euclidean distance

$$
R=\left(\sum_{i=1}^{n_{\mathrm{var}}}\left|x_{i}-y_{i}\right|^{2}\right)^{\frac{1}{2}}
$$

where $n_{\text {var }}$ is the number of input variables used for the classification, $x_{i}$ are coordinates of an event from a training sample and $y_{i}$ are variables of an observed test event. The $k$ events with the smallest values of $R$ are the $k$-nearest neighbours. The value of $k$ determines the size of the neighbourhood for which a probability density function is evaluated. Large values of $k$ do not capture the local behavior of the probability density function. On the other hand, small values of $k$ cause statistical fluctuations in the probability density estimate. A case study with real data suggests that values of $k$ between 10 and 100 are appropriate and result in similar classification performance when the training sample contains hundreds of thousands of events (and $n_{\mathrm{var}}$ is of the order of a few variables).

The classification algorithm finds k-nearest training events around a query point

$$
k=k_{S}+k_{B},
$$



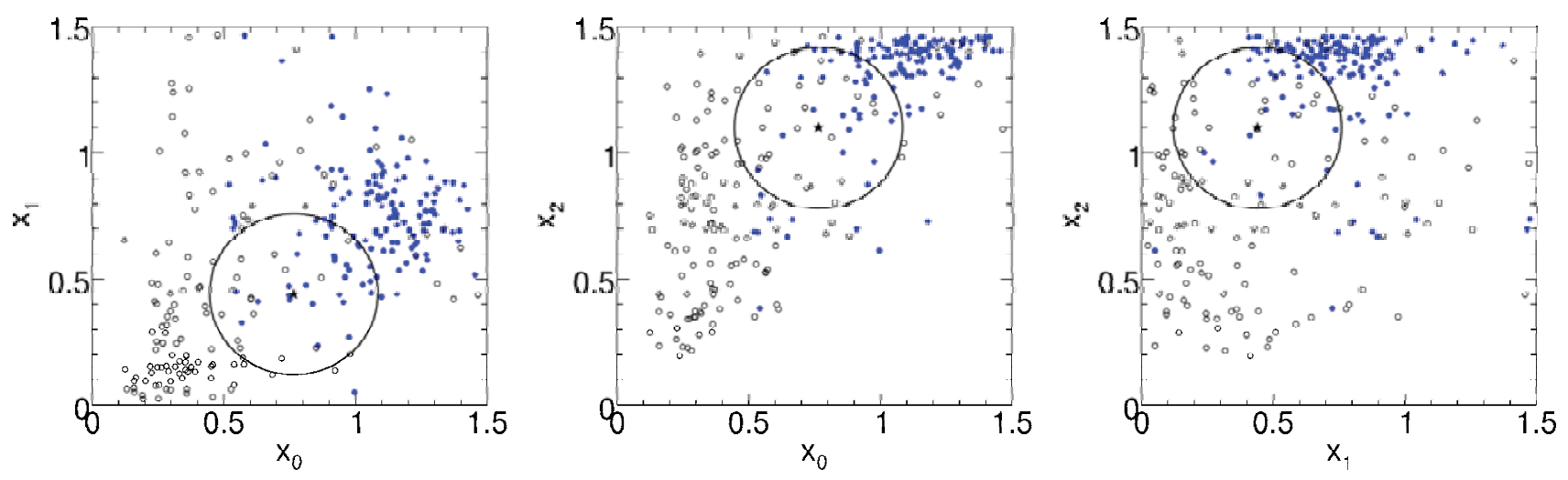

FIG. 12: Example for the k-nearest neighbour algorithm in a three-dimensional space (i.e., for three discriminating input variables). The three plots are projections upon the two-dimensional coordinate planes. The full (open) circles are the signal (background) events. The k-NN algorithm searches for 20 nearest points in the nearest neighborhood (circle) of the query event, shown as a star. The nearest neighborhood counts 13 signal and 7 background points so that query event may be classified as a signal candidate.

where $k_{S(B)}$ is number of the signal (background) events in the training sample. The relative probability that the test event is of signal type is given by

$$
P_{S}=\frac{k_{S}}{k_{S}+k_{B}}=\frac{k_{S}}{k} .
$$

The choice of the metric governs the performance of the nearest neighbour algorithm. When input variables have different units a variable that has a wider distribution contributes with a greater weight to the Euclidean metric. This feature is compensated by rescaling the variables using a user-defined scaling fraction. The scaling factor applied to variable $i$ is determined by the width $w_{i}$ of the $x_{i}$ distribution for the combined sample of signal and background events : $w_{i}$ is the interval that contains the defined fraction of $x_{i}$ training values. The input variables are then rescaled by $1 / w_{i}$, leading to the rescaled metric

$$
R_{\text {rescaled }}=\left(\sum_{i=1}^{d} \frac{1}{w_{i}^{2}}\left|x_{i}-y_{i}\right|^{2}\right)^{\frac{1}{2}} .
$$

Figure 12 shows an example of event classification with the k-nearest neighbour algorithm. ${ }^{17}$

The output of the $k$-nearest neighbour algorithm can be optionally interpreted as a probability that an event is of signal type, if the numbers (better : sum of event weights) of signal and background events in the training sample are equal. If set training events of the overabundant type are randomly removed until parity is achieved.

Like (more or less) all TMVA classifiers, the k-nearest neighbour estimate suffers from statistical fluctuations in the training data. The typically high variance of the $\mathrm{k}-\mathrm{NN}$ response is mitigated by adding a weight function that depends smoothly on the distance from a test event. The current k-NN implementation uses a polynomial kernel

$$
W(x)= \begin{cases}\left(1-|x|^{3}\right)^{3} & \text { if }|x|<1 \\ 0 & \text { otherwise }\end{cases}
$$

If $R_{k}$ is the distance between the test event and the $k$ th neighbour, the events are weighted according to the formula:

$$
W_{S(B)}=\sum_{i=1}^{k_{S(B)}} W\left(\frac{R_{i}}{R_{k}}\right)
$$

\footnotetext{
17 The number of training events shown has been greatly reduced to illustrate the principle of the algorithm. In a real application a typical $\mathrm{k}-\mathrm{NN}$ training sample should be amplc.
} 
where $k_{S(B)}$ is number of the signal (background) events in the neighbourhood. The weighted signal probability for the test event is then given by

$$
P_{S}=\frac{W_{S}}{W_{S}+W_{B}}
$$

The k-NN algorithm in TMVA also implements a simple multi-dimensional (multi-target) regression model. For a test event, the algorithm finds the k-nearest neighbours using the input variables, where each training event contains a regression value. The predicted regression value for the test event is the weighted average of the regression values of the k-nearest neighbours, cf.Eq. (36) on page 27.

\section{Performance}

The simplest implementation of the $\mathrm{k}$-NN algorithm would store all training events in an array. The classification would then be performed by looping over all stored events and finding the k-nearest neighbours. As discussed in Sec. 3.2, such an implementation is impractical for large training samples. The k-NN algorithm therefore uses a $k d$-tree structure [14] that significantly improves the performance.

The TMVA implementation of the $k$-NN method is reasonably fast to allow classification of large data sets. In particular, it is faster than the adaptive PDE-RS method (cf. Sec. 7.3). Note that the k-NN method is not appropriate for problems where the number of input variables exceeds $n_{\text {var }} \gtrsim 10$. The neighbourood size $R$ depends on $n_{\text {var }}$ and the size of the training sample $N$ as

$$
R_{N} \propto \frac{1}{\sqrt[n_{\operatorname{var}}]{N}} .
$$

A large training set allows the algorithm to probe small-scale features that distinguish signal and background events.

\subsection{H-Matrix discriminant}

The origins of the H-Matrix approach dates back to works of Fisher and Mahalanobis in the context of Gaussian classifiers $[24,25]$. It discriminates one class (signal) of a feature vector from another (background). The correlated elements of the vector are assumed to be Gaussian distributed, and the inverse of the covariance matrix is the $H$-Matrix. A multivariate $\chi^{2}$ estimator is built that exploits differences in the mean values of the vector elements between the two classes for the purpose of discrimination.

The H-Matrix classifier as it is implemented in TMVA is equal or less performing than the Fisher discriminant (see Sec. 7.7), and has been only included for completeness.

For an event $i$, each one $\chi^{2}$ estimator $\left(\chi_{S(B)}^{2}\right)$ is computed for signal $(S)$ and background $(B)$, using estimates for the sample means $\left(\bar{x}_{S(B), k}\right)$ and covariance matrices $\left(C_{S(B)}\right)$ obtained from the training data

$$
\chi_{U}^{2}(i)=\sum_{k, \ell=1}^{n_{\text {var }}}\left(x_{k}(i)-\bar{x}_{U, k}\right) C_{U, k \ell}^{-1}\left(x_{\ell}(i)-\bar{x}_{U, \ell}\right),
$$

where $U=S, B$. From this, the discriminant

$$
y_{H}(i)=\frac{\chi_{B}^{2}(i)-\chi_{S}^{2}(i)}{\chi_{B}^{2}(i)+\chi_{S}^{2}(i)}
$$

is computed to discriminate between the signal and background classes.

\section{Performance}

The TMVA implementation of the H-Matrix classifier has been shown to underperform in comparison with the corresponding Fisher discriminant (cf. Sec. 7.7), when using similar assumptions and complexity. It might therefore be considered to be depreciated. 


\subsection{Fisher discriminants (linear discriminant analysis)}

In the method of Fisher discriminants [24] event selection is performed in a transformed variable space with zero linear correlations, by distinguishing the mean values of the signal and background distributions. The linear discriminant analysis determines an axis in the (correlated) hyperspace of the input variables such that, when projecting the output classes (signal and background) upon this axis, they are pushed as far as possible away from each other, while events of a same class are confined in a close vicinity. The linearity property of this classifier is reflected in the metric with which "far apart" and "close vicinity" are determined : the covariance matrix of the discriminating variable space.

The classification of the events in signal and background classes relies on the following characteristics : the overall sample means $\bar{x}_{k}$ for each input variable $k=1, \ldots, n_{\text {var }}$, the class-specific sample means $\bar{x}_{S(B), k}$, and total covariance matrix $C$ of the sample. The covariance matrix can be decomposed into the sum of a within- $(W)$ and a between-class matrix $(B)$. They respectively describe the dispersion of events relative to the means of their own class (within-class matrix), and relative to the overall sample means (between-class matrix) ${ }^{18}$.

The Fisher coefficients, $F_{k}$, are then given by

$$
F_{k}=\frac{\sqrt{N_{S} N_{B}}}{N_{S}+N_{B}} \sum_{\ell=1}^{n_{\mathrm{var}}} W_{k \ell}^{-1}\left(\bar{x}_{S, \ell}-\bar{x}_{B, \ell}\right)
$$

where $N_{S(B)}$ are the number of signal (background) events in the training sample. The Fisher discriminant $y_{\mathrm{Fi}}(i)$ for event $i$ is given by

$$
y_{\mathrm{Fi}}(i)=F_{0}+\sum_{k=1}^{n_{\mathrm{var}}} F_{k} x_{k}(i) .
$$

The offset $F_{0}$ centers the sample mean $\bar{y}_{\text {Fi }}$ of all $N_{S}+N_{B}$ events at zero.

Instead of using the within-class matrix, the Mahalanobis variant determines the Fisher coefficients as follows [25]

$$
F_{k}=\frac{\sqrt{N_{S} N_{B}}}{N_{S}+N_{B}} \sum_{\ell=1}^{n_{\mathrm{var}}} C_{k \ell}^{-1}\left(\bar{x}_{S, \ell}-\bar{x}_{B, \ell}\right),
$$

where $C_{k \ell}=W_{k \ell}+B_{k \ell}$.

The Fisher discriminant analysis aims at simultaneously maximising the between-class separation while minimising the within-class dispersion. A useful measure of the discrimination power of a variable is therefore given by the diagonal quantity $B_{k k} / C_{k k}$, which is used for the ranking of the input variables.

\section{Performance}

In spite of the simplicity of the classifier, Fisher discriminants can be competitive with likelihood and nonlinear discriminants in certain cases. In particular, Fisher discriminants are optimal for Gaussian distributed variables with linear correlations (cf. the standard toy example that comes with TMVA).

On the other hand, no discrimination at all is achieved when a variable has the same sample mean for signal and background, even if the shapes of the distributions are very different. Thus, Fisher discriminants often benefit from suitable transformations of the input variables. For example, if a variable $x \in[-1,1$ : has a a signal distributions of the form $x^{2}$, and a uniform background distributions, their mean value

${ }^{18}$ The within-class matrix is given by

$$
W_{k \ell}=\sum_{U-S, B}\left\langle x_{U, k} \quad \bar{x}_{U, k}\right\rangle\left\langle x_{U, \ell} \quad \bar{x}_{U, \ell}\right\rangle=C_{S, k \ell} \mid C_{B, k \ell}:
$$

where $C_{S(B)}$ is the covariance matrix of the signal (background) sample. The between-class matrix is obtained by

$$
B_{k \ell}=\frac{1}{2} \sum_{U=S, B}\left(\bar{x}_{U, k} \quad \bar{x}_{k}\right)\left(\bar{x}_{U, \ell} \cdots \bar{x}_{\ell}\right)
$$

where $\bar{x}_{S(B), k}$ is the average of variabie $x_{k}$ for the signal (background) sample, and $\bar{x}_{k}$ denotes the average for the entire sample. 
is zero in both cases, leading to no separation. The simple transformation $x \rightarrow|x|$ renders this variable powerful for the use in a Fisher discriminant.

\subsection{Linear discriminant analysis (LD)}

The linear discriminant analysis provides data classification using a linear model, where linear refers to the discriminant function $y(\mathbf{x})$ being linear in the parameters $\beta$

$$
y(\mathbf{x})=\mathbf{x}^{\top} \beta+\beta_{0},
$$

where $\beta_{0}$ (denoted the bias) is adjusted so that $y(\mathbf{x}) \geq 0$ for signal and $y(\mathbf{x})<0$ for background. It can be shown that this is equivalent to the Fisher discriminant, which seeks to maximise the ratio of between-class variance to within-class variance by projecting the data onto a linear subspace.

Assuming that there are $m+1$ parameters $\beta_{0}, \cdots, \beta_{m}$ to be estimated using a training set comprised of $n$ events, the defining equation for $\beta$ is

$$
Y=X \beta,
$$

where we have absorbed $\beta_{0}$ into the vector $\beta$ and introduced the matrices

$$
Y=\left(\begin{array}{c}
y_{1} \\
y_{2} \\
\vdots \\
y_{n}
\end{array}\right) \text { and } X=\left(\begin{array}{cccc}
1 & x_{11} & \cdots & x_{1 m} \\
1 & x_{21} & \cdots & x_{2 m} \\
\vdots & \vdots & \ddots & \vdots \\
1 & x_{n 1} & \cdots & x_{n m}
\end{array}\right)
$$

where the constant column in $X$ represents the bias $\beta_{0}$ and $Y$ is composed of the target values with $y_{i}=1$ if the $i$ th event belongs to the signal class and $y_{i}-0$ if the $i$ th event belongs to the background class. Applying the method of least squares, we now obtain the normal equations for the classification problem, given by

$$
X^{T} X \beta=X^{T} Y \Longleftrightarrow \beta=\left(X^{T} X\right)^{-1} X^{T} Y .
$$

The transformation $\left(X^{T} X\right)^{-1} X^{T}$ is known as the Moore-Penrose pseudo inverse of $X$ and can be regarded as a generalisation of the matrix inverse to non-square matrices. It requires that the matrix $X$ has full rank.

If weighted events are used, this is simply taken into account by introducing a diagonal weight matrix $W$ and modifying the normal equations as follows:

$$
\beta=\left(X^{T} W X\right)^{-1} X^{T} W Y .
$$

Considering two events $\mathbf{x}_{1}$ and $\mathbf{x}_{2}$ on the decision boundary, we have $y\left(\mathbf{x}_{1}\right)=y\left(\mathbf{x}_{2}\right)=0$ and hence $\left(\mathrm{x}_{1}-\mathrm{x}_{2}\right)^{T} \beta=0$. Thus we see that the $\mathrm{LD}$ can be geometrically interpreted as determining the decision boundary by finding an orthogonal vector $\beta$.

The implementation of LD provides a ranking of the input variables based on the coefficients of the variables in the linear combination that forms the decision boundary. The order of importance of the discriminating variables is assumed to agree with the order of the absolute values of the coefficients.

It is straightforward to apply the LD algorithm to linear regression by replacing the binary targets $y_{i} \in 0,1$ in the training data with the measured values of the function which is to be estimated. The resulting function $y(\mathbf{x})$ is then the best estimate for the data obtained by least-squares regression.

\section{Performance}

The LD is optimal for Gaussian distributed variables with linear correlations (cf.the standard toy example that comes with TMVA) and can be competitive with likelihood and nonlinear discriminants in certain cases. No discrimination is achieved when a variable has the same sample mean for signal and background, but the LD can often benefit from suitable transformations of the input variables. For example, if a variable $x \in[-1,1]$ has a signal distribution of the form $x^{2}$ and a uniform background 
distribution, their mean value is zero in both cases, leading to no separation. The simple transformation $x \rightarrow|x|$ renders this variable powerful for the use with $L D$.

\subsection{Function discriminant analysis (FDA)}

The common goal of all TMVA discriminators is to determine an optimal separating function in the multivariate space of all input variables. The Fisher discriminant solves this analytically for the linear case, while artificial neural networks, support vector machines or boosted decision trees provide nonlinear approximations with - in principle - arbitrary precision if enough training statistics is available and the chosen architecture is flexible enough.

The function discriminant analysis (FDA) provides an intermediate solution to the problem with the aim to solve relatively simple or partially nonlinear problems. The user provides the desired function with adjustable parameters via the configuration option string, and FDA fits the parameters to it, requiring the function value to be as close as possibie to the real value (to 1 for signal and 0 for background in classification). Its advantage over the more involved and automatic nonlinear discriminators is the simplicity and transparency of the discrimination expression. A shortcoming is that FDA will underperform for involved problems with complicated, phase space dependent nonlinear correlations.

A typical option string to defined FDA could look as follows :

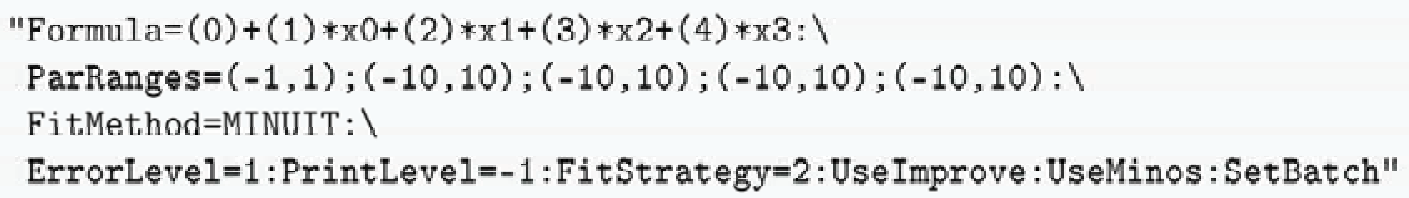

Code Example 2: FDA booking option example simulating a linear Fisher discriminant (cf. Sec. 7.7). The top line gives the discriminator expression, where the $x i$ denote the input variables and the $(j)$ denote the coefficients to be determined by the fit. Allowed are all standard functions and expressions, including the functions belonging to the ROOT TMath library. The second line determines the limits for the fit parameters, where the numbers of intervals given must correspond to the number of fit parameters defined. The third line defines the fitter to be used (here Minuit), and the last line is the fitter configuration.

For simple formula with a single global fit solution, Minuit will be the most efficient fitter. However, if the problem is complicated, highly nonlinear, and/or has a non-unique solution space, more involved fitting algorithms may be required. In that case the Genetic Algorithm combined or not with a Minuit converger should lead to the best results. After fit convergence, FDA prints the fit results (parameters and estimator value) as well as the discriminator expression used on standard output. The smaller the estimator value, the better the solution found. The normalised estimator is given by

$$
\begin{aligned}
& \text { For classification : } \mathcal{E}=\frac{1}{W_{S}} \sum_{i=1}^{N_{S}}\left(F\left(\mathbf{x}_{i}\right)-1\right)^{2} w_{i}+\frac{1}{W_{B}} \sum_{i=1}^{N_{B}} F^{2}\left(\mathbf{x}_{i}\right) w_{i}, \\
& \text { For regression : } \quad \mathcal{E}=\frac{1}{W} \sum_{i=1}^{N}\left(F\left(\mathbf{x}_{i}\right)-\mathbf{t}_{i}\right)^{2} w_{i}
\end{aligned}
$$

where for classification the first (second) sum is over the signal (background) training events, and for regression it is over all training events, $F\left(\mathbf{x}_{i}\right)$ is the discriminator function, $\mathbf{x}_{i}$ is the tuple of the $n_{\text {var }}$ input variables for event $i, w_{i}$ is the event weight, $\mathbf{t}_{i}$ the tuple of training regression targets, $W_{S(B)}$ is the sum of all signal (background) weights in the training sample, and $W$ the sum over all training weights.

\section{Performance}

The FDA performance depends on the complexity and fidelity of the user-defined discriminator function. As a general rule, it should be able to reproduce the discrimination power of any linear discriminant analysis. To reach into the nonlinear domain, it is useful to inspect the correlation profiles of the input variables, and add quadratic and higher polynomial terms between variables as necessary. Comparison with more involved nonlinear classifiers can be used as a guide. 


\subsection{Artificial Neural Networks (nonlinear discriminant analysis)}

An Artificial Neural Network (ANN) is most generally speaking any simulated collection of interconnected neurons, with each neuron producing a certain response at a given set of input signals. By applying an external signal to some (input) neurons the network is put into a defined state that can be measured from the response of one or several (output) neurons. One can therefore view the neural network as a mapping from a space of input variables $x_{1}, \ldots, x_{n_{\mathrm{var}}}$ onto a one-dimensional (e.g. in case of a signalversus-background discrimination problem) or multi-dimensional space of output variables $y_{1}, \ldots, y_{\mathrm{m}_{\mathrm{var}}}$. The mapping is nonlinear if at least one neuron has a nonlinear response to its input.

In TMVA three neural network implementations are available to the user. The first was adapted from a FORTRAN code developed at the Université Blaise Pascal in Clermont-Ferrand, ${ }^{19}$ the second is the ANN implementation that comes with ROOT. The third is a newly developed neural network (denoted $M L P$ ) that is faster and more flexible than the other two and is the recommended neural network to use with TMVA. All three neural networks are feed-forward multilayer perceptrons.

The TMVA implementation of MLP supports random and importance event sampling. With event sampling enabled, only a fraction of the training events is used for the training of the MLP. The events can be either selected randomly or the same events are sampled again depending on the training performance achieved for classification or regression. If for a given set of events the training leads to a decrease of the error of the test sample, the probability for the events of being selected decreases, while it increases otherwise. The aim of random and importance sampling is foremost to speed-up the training for large training samples. As a side effect, random or importance sampling may also increase the robustness of the training algorithm with respect to convergence in a local minimum.

The behaviour of an artificial neural network is determined by the layout of the neurons, the weights of the inter-neuron connections, and by the response of the neurons to the input, described by the neuron response function $\rho$.

\section{Multilayer Perceptron}

While in principle a neural network with $n$ neurons can have $n^{2}$ directional connections, the complexity can be reduced by organising the neurons in layers and only allowing direct connections from a given layer to the following layer (see Fig. 13). This kind of neural network is termed multi-layer perceptron; all neural net implementations in TMVA are of this type. The first layer of a multilayer perceptron is the input layer, the last one the output layer, and all others are hidden layers. For a classification problem with $n_{\mathrm{var}}$ input variables the input layer consists of $n_{\text {var }}$ neurons that hold the input values, $x_{1}, \ldots, x_{n_{\mathrm{var}}}$, and one neuron in the output layer that holds the output variable, the neural net estimator $y_{\text {ANN }}$.

For a regression problem the network structure is similar, except that for multi-target regression each of the targets is represented by one output neuron. A weight is associated to each directional connection between the output of one neuron and the input of another neuron. When calculating the input value to the response function of a neuron, the output values of all neurons connected to the given neuron are multiplied with theses weights.

The neuron response function $\rho$ maps the neuron input $i_{1}, \ldots, i_{n}$ onto the neuron output (Fig. 14). Often it can be separated into a $\mathcal{R}^{n} \mapsto \mathcal{R}$ synapse function $\kappa$, and a $\mathcal{R} \mapsto \mathcal{R}$ neuron activation function $\alpha$, so that $\rho-\alpha \circ \kappa$. The functions $\kappa$ and $\alpha$ can have the following forms :

$$
\kappa:\left(y_{1}^{(\ell)}, . ., y_{n}^{(\ell)} \mid w_{0 j}^{(\ell)}, . ., w_{n j}^{(\ell)}\right) \rightarrow \begin{cases}w_{0 j}^{(\ell)}+\sum_{i=1}^{n} y_{i}^{(\ell)} w_{i j}^{(\ell)} & \text { Sum }, \\ w_{0 j}^{(\ell)}+\sum_{i=1}^{n}\left(y_{i}^{(\ell)} w_{i j}^{(\ell)}\right)^{2} & \text { Sum of squares }, \\ w_{0 j}^{(\ell)}+\sum_{i=1}^{n}\left|y_{i}^{(\ell)} w_{i j}^{(\ell)}\right| & \text { Sum of absolutes },\end{cases}
$$

${ }^{19}$ The original Clermont-Ferrand neural network has been used for Higgs search analyses in ALEPH, and background fighting in rare $B$-decay searches by the BABAR. Collaboration. For the use in TMVA the FORTRAN code has been converted to $\mathrm{C}++$. 


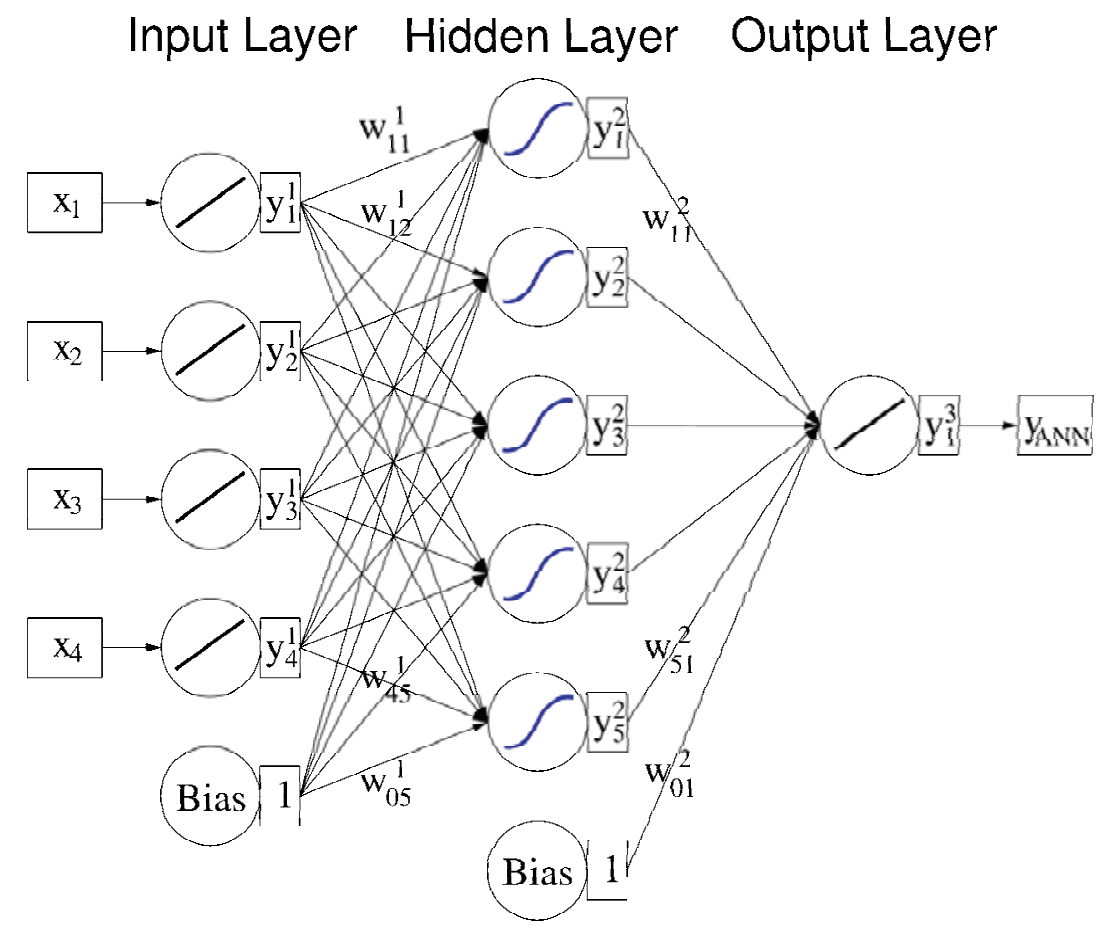

FIG. 13: Multilayer perceptron with one hidden layer.

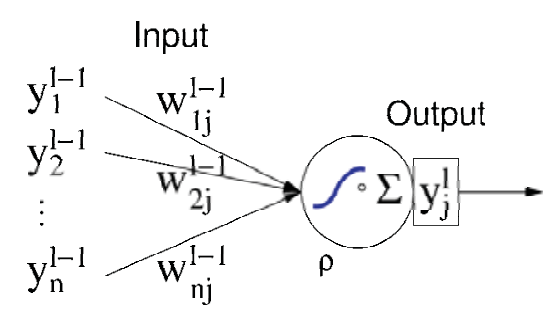

FIG. 14: Single neuron $j$ in layer $\ell$ with $n$ input connections. The incoming connections carry a weight of $w_{i j}^{(l-1)}$.

$$
\alpha: x \rightarrow \begin{cases}x & \text { Linear }, \\ \frac{1}{1+e^{-k x}} & \text { Sigmoid. } \\ \frac{e^{x}-e^{-x}}{e^{x}+e^{-x}} & \text { Tanh }, \\ e^{-x^{2} / 2} & \text { Radial. }\end{cases}
$$

\section{Network architecture}

The number of hidden layers in a network and the number of neurons in these layers are user defined. For example the configuration "HiddenLayers $=\mathrm{N}-1, \mathbb{N}+10,3$ " creates a network with three hidden layers, the first hidden layer with $n_{\mathrm{var}}-1$ neurons, the second with $n_{\mathrm{var}}+10$ neurons, and the third with 3 neurons.

When building a network two rules should be kept in mind. The first is the theorem by Weierstrass, which if applied to neural nets, ascertains that for a multilayer perceptron a single hidden layer is sufficient to approximate a given continuous correlation function to any precision, provided that a sufficiently large number of neurons is used in the hidden layer. If the available computing power and the size of the training data sample suffice, one can increase the number of neurons in the hidden layer until the optimal performance is reached.

It is likely that the same performance can be achieved with a network of more than one hidden layer and a potentially much smaller total number of hidden neurons. This would lead to a shorter training 
time and a more robust network.

\section{Training of the neural network}

The most common algorithm for adjusting the weights that optimise the classification performance of a neural network is the so-called back propagation. It belongs to the family of supervised learning methods, where the desired output for every input event is known. Back propagation is used by all neural networks in TMVA. The output of a network (here for simplicity assumed to have a single hidden layer with a Tanh activation function, and a linear activation function in the output layer) is given by

$$
y_{\mathrm{ANN}}=\sum_{j=1}^{n_{\mathrm{h}}} y_{j}^{(2)} w_{j 1}^{(2)}=\sum_{j=1}^{n_{\mathrm{h}}} \tanh \left(\sum_{i=1}^{n_{\mathrm{var}}} x_{i} w_{i j}^{(1)}\right) \cdot w_{j 1}^{(2)},
$$

where $n_{\mathrm{var}}$ and $n_{\mathrm{h}}$ are the number of neurons in the input layer and in the hidden layer, respectively, $w_{i j}^{(1)}$ is the weight between input-layer neuron $i$ and hidden-layer neuron $j$, and $w_{j 1}^{(2)}$ is the weight between the hidden-layer neuron $j$ and the output neuron. A simple sum was used in Eq. (58) for the synapse function $\kappa$.

During the learning process the network is supplied with $N$ training events $\mathbf{x}_{a}=\left(x_{1}, \ldots, x_{n_{\text {var }}}\right)_{a}$, $a=1, \ldots, N$. For each training event $a$ the neural network output $y_{\mathrm{ANN}, a}$ is computed and compared to the desired output $\hat{y}_{a} \in\{1,0\}$ (in classification 1 for signal events and 0 for background events). An error function $E$, measuring the agreement of the network response with the desired one, is defined by

$$
E\left(\mathbf{x}_{1}, \ldots, \mathbf{x}_{N} \mid \mathbf{w}\right)=\sum_{a=1}^{N} E_{a}\left(\mathbf{x}_{a} \mid \mathbf{w}\right)=\sum_{a=1}^{N} \frac{1}{2}\left(y_{\mathrm{ANN}, a}-\hat{y}_{a}\right)^{2},
$$

where $\mathbf{w}$ denotes the ensemble of adjustable weights in the network. The set of weights that minimises the error function can be found using the method of steepest or gradient descent, provided that the neuron response function is differentiable with respect to the input weights. Starting from a random set of weights $\mathbf{w}^{(\rho)}$ the weights are updated by moving a small distance in $\mathbf{w}$-space into the direction $-\nabla_{\mathbf{w}} E$ where $E$ decreases most rapidly

$$
\mathbf{w}^{(\rho+1)}=\mathbf{w}^{(\rho)}-\eta \nabla_{\mathbf{w}} E,
$$

where the positive number $\eta$ is the learning rate.

The weights connected with the output layer are updated by

$$
\Delta w_{j 1}^{(2)}=-\eta \sum_{a=1}^{N} \frac{\partial E_{a}}{\partial w_{j 1}^{(2)}}=-\eta \sum_{a=1}^{N}\left(y_{\mathrm{ANN}, a}-\hat{y}_{a}\right) y_{j, a}^{(2)},
$$

and the weights connected with the hidden layers are updated by

$$
\Delta w_{i j}^{(1)}=-\eta \sum_{a=1}^{N} \frac{\partial E_{a}}{\partial w_{i j}^{(1)}}=-\eta \sum_{a=1}^{N}\left(y_{\mathrm{ANN}, a}-\hat{y}_{a}\right) y_{j, a}^{(2)}\left(1-y_{j, a}^{(2)}\right) w_{j 1}^{(2)} x_{i, a},
$$

where we have used $\tanh ^{\prime} x=\tanh x(1-\tanh x)$. This method of training the network is denoted bulk learning, since the sum of errors of all training events is used to update the weights. An alternative choice is the so-called online learning, where the update of the weights occurs at each event. The weight updates are obtained from Eqs. (61) and (62) by removing the event summations. In this case it is important to use a well randomised training sample. Online learning is the learning method implemented in TMVA.

The Broyden-Fletcher-Goldfarb-Shannon (BFGS) method [26] differs from back propagation by the use of second derivatives of the error function to adapt the synapse weight by an algorithm which is composed of four main steps.

1. Two vectors, $D$ and $Y$ are calculated. The vector of weight changes $D$ represents the evolution between one iteration of the algorithm $(k-1)$ to the next $(k)$. Each synapse weight corresponds to 
one element of the vector. The vector $Y$ is the vector of gradient errors.

$$
\begin{aligned}
& D_{i}^{(k)}=w_{i}^{(k)}-w_{i}^{(k-1)}, \\
& Y_{i}^{(k)}=g_{i}^{(k)}-g_{i}^{(k-1)},
\end{aligned}
$$

where $i$ is the synapse index, $g_{i}$ is the $i$-th synapse gradient, ${ }^{20} w_{i}$ is the weight of the $i$-th synapse, and $k$ denotes the iteration counter.

2. Approximate the inverse of the Hessian matrix, $H^{-1}$, at iteration $k$ by

$$
H^{-1(k)}=\frac{D \cdot D^{T} \cdot\left(1+Y^{T} \cdot I^{-1(k-1)} \cdot Y\right)}{Y^{T} \cdot D}-D \cdot Y^{T} \cdot H+H \cdot Y \cdot D^{T}+H^{-1(k-1)},
$$

where superscripts $(k)$ are implicit for $D$ and $Y$.

3. Estimate the vector of weight changes by

$$
D^{(k)}=-H^{-1(k)} \cdot Y^{(k)} .
$$

4. Compute a new vector of weights by applying a line search algorithm. In the line search the error function is locally approximated by a parabola. The algorithm evaluates the second derivatives and determines the point where the minimum of the parabola is expected. The total error is evaluated for this point. The algorithm then evaluates points along the line defined by the direction of the gradient in weights space to find the absolute minimum. The weights at the minimum are used for the next iteration. The learning parameter, which defines by how much the weights are changed in one epoch along the line where the minimum is suspected, is multiplied with the learning rate as long as the training error of the neural net with the changed weights is below the one with unchanged weights. If the training error of the changed neural net were already larger for the initial learning parameter, it is divided by the learning rate until the training error becomes smaller. The iterative and approximate calculation of $H^{-1(k)}$ turns less accurate with an increasing number of iterations. The matrix is therefore reset to the unit matrix every user-defined number of steps.

The advantage of the BFGS method compared to BG is the smaller number of iterations. However, because the computing time for one iteration is proportional to the squared number of synapses, large networks are particularly penalised.

\section{Variable ranking}

The MLP neural network implements a variable ranking that uses the sum of the weights-squared of the connections between the variable's neuron in the input layer and the first hidden layer. The importance $I_{i}$ of the input variable $i$ is given by

$$
I_{i}=\bar{x}_{i}^{2} \sum_{j=1}^{n_{\mathrm{h}}}\left(w_{i j}^{(i)}\right)^{2}, \quad i=1, \ldots, n_{\mathrm{var}},
$$

where $\bar{x}_{i}$ is the sample mean of input variable $i$.

\section{Performance}

In the tests we have carried out so far, the MLP and ROOT networks performed equally well, however with a clear speed advantage for the MLP. The Clermont-Ferrand neural net exhibited worse classification performance in these tests, which is partly due to the slow convergence of its training (at least 10k training cycles are required to achieve approximately competitive results).

${ }^{20}$ The synapse gradient is estimated in the same way as in the BP method (with initial gradient and weights set to zero). 


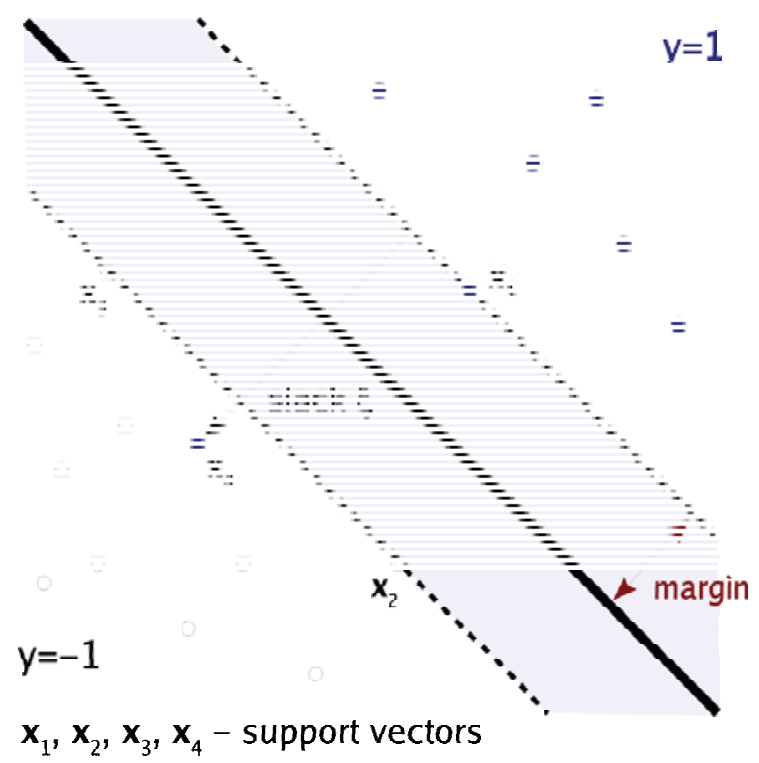

FIG. 15: Hyperplane classifier in two dimensions. The vectors (events) $\mathbf{x}_{1-4}$ define the hyperplane and margin, i.e., they are the support vectors.

\subsection{Support Vector Machine (SVM)}

In the early 1960 s a linear support vector method has been developed for the construction of separating hyperplanes for pattern recognition problems [39, 40]. It took 30 years before the method was generalised to nonlinear separating functions [41, 42] and for estimating real-valued functions (regression) [43]. At that moment it became a general purpose algorithm, performing classification and regression tasks which can compete with neural networks and probability density estimators. Typical applications of SVMs include text categorisation, character recognition, bio-informatics and face detection.

The main idea of the SVM approach to classification problems is to build a hyperplane that separates signal and background vectors (events) using only a minimal subset of all training vectors (support vectors). The position of the hyperplane is obtained by maximizing the margin (distance) between it and the support vectors. The extension to nonlinear SVMs is performed by mapping the input vectors onto a higher dimensional feature space in which signal and background events can be separated by a linear procedure using an optimally separating hyperplane. The use of kernel functions eliminates thereby the explicit transformation to the feature space and simplifies the computation.

The implementation of the newiy introduced regression is similar to the approach in classification. It also maps input data into higher dimensional space using previously chosen support vectors. Instead of separating events of two types, it determines the hyperplane with events of the same value (which is equal to the mean from all training events). The final value is estimated based on the distance to the hyperplane which is computed by the selected kernel function.

A detailed description of the SVM formalism can be found, for example, in Ref. [44]. Here only a brief introduction of the TMVA implementation is given.

\section{Linear SVM}

Consider a simple two-class classifier with oriented hyperplanes. If the training data is linearly separable, a vector-scalar pair $(\vec{w}, b)$ can be found that satisfies the constraints

$$
y_{i}\left(\overrightarrow{x_{i}} \cdot \vec{w}+b\right)-1 \geq 0, \quad \forall_{i},
$$

where $\vec{x}_{i}$ are the input vectors, $y_{i}$ the desired outputs $\left(y_{i}= \pm 1\right)$, and where the pair $(\vec{w}, b)$ defines a hyperplane. The decision function of the classifier is $f\left(\vec{x}_{i}\right)=\operatorname{sign}\left(\vec{x}_{i} \cdot \vec{w}+b\right)$, which is +1 for all points on one side of the hyperplane and -1 for the points on the other side. 
Intuitively, the classifier with the largest margin will give better separation. The margin for this linear classifier is just $2 /|\vec{w}|$. Hence to maximise the margin, one needs to minimise the cost function $W=|\vec{w}|^{2} / w$ with the constraints from Eq. (68).

At this point it is beneficial to consider the significance of different input vectors $\vec{x}_{i}$. The training events lying on the margins, which are called the support vectors (SV), are the events that contribute to defining the decision boundary (see Fig. 15). Hence if the other events are removed from the training sample and the classifier is retrained on the remaining events, the training will result in the same decision boundary. To solve the constrained quadratic optimisation problem, we first reformulate it in terms of a Lagrangian

$$
\mathcal{L}(\vec{w}, b, \vec{\alpha})=\frac{1}{2}|\vec{w}|^{2}-\sum_{i} \alpha_{i}\left(y_{i}\left(\left(\vec{x}_{i} \cdot \vec{w}\right)+b\right)-1\right)
$$

where $\alpha_{i} \geq 0$ and the condition from Eq. (68) must be fulfilled. The Lagrangian $\mathcal{L}$ is minimised with respect to $\vec{w}$ and $b$ and maximised with respect to $\vec{\alpha}$. The solution has an expansion in terms of a subset of input vectors for which $\alpha_{i} \neq 0$ (the support vectors) :

$$
\vec{w}=\sum_{i} \alpha_{i} y_{i} \vec{x}_{i}
$$

because $\partial \mathcal{L} / \partial b=0$ and $\partial \mathcal{L} / \partial \vec{w}=0$ hold at the extremum. The optimisation problem translates to finding the vector $\vec{\alpha}$ which maximises

$$
\mathcal{L}(\vec{\alpha})=\sum_{i} \alpha_{i}-\frac{1}{2} \sum_{i j} \alpha_{i} \alpha_{j} y_{i} y_{j} \vec{x}_{i} \cdot \vec{x}_{j}
$$

Both the optimisation problem and the final decision function depend only on scalar products between input vectors, which is a crucial property for the generalisation to the nonlinear case.

\section{Nonseparable data}

The above algorithm can be extended to non-separable data. The classification constraints in Eq. (68) are modified by adding a "slack" variable $\xi_{i}$ to it $\left(\xi_{i}=0\right.$ if the vector is properly classified, otherwise $\xi_{i}$ is the distance to the decision hyperplane)

$$
y_{i}\left(\overrightarrow{x_{i}} \cdot \vec{w}+b\right)-1+\xi_{i} \geq 0, \quad \xi_{i} \geq 0, \quad \forall_{i} .
$$

This admits a certain amount of misclassification. The training algorithm thus minimises the modified cost function

$$
W=\frac{1}{2}|\vec{w}|^{2}+C \sum_{i} \xi_{i}
$$

describing a trade-off between margin and misclassification. The cost parameter $C$ sets the scale by how much misclassification increases the cost function.

\section{Nonlinear SVM}

The SVM formulation given above can be further extended to build a nonlinear SVM which can classify

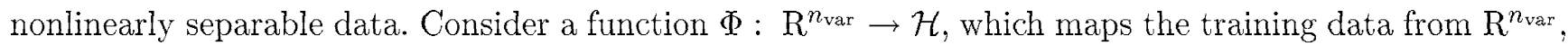
where $n_{\text {var }}$ is the number of discriminating input variables, to some higher dimensional space $\mathcal{H}$. In the $\mathcal{H}$ space the signal and background events can be linearly separated so that the linear SVM formulation can be applied. We have seen in Eq. (71) that event variables only appear in the form of scalar products $\vec{x}_{i} \cdot \vec{x}_{j}$, which become $\Phi\left(\vec{x}_{i}\right) \cdot \Phi\left(\vec{x}_{j}\right)$ in the higher dimensional feature space $\mathcal{H}$. The latter scalar product can be approximated by a kernel function

$$
K\left(\vec{x}_{i}, \vec{x}_{j}\right) \approx \Phi\left(\vec{x}_{i}\right) \cdot \Phi\left(\vec{x}_{j}\right)
$$


which avoids the explicit computation of the mapping function $\Phi(\vec{x})$. This is desirable because the exact form of $\Phi(\vec{x})$ is hard to derive from the training data. Most frequently used kernel functions are

$$
\begin{aligned}
& K(\vec{x}, \vec{y})=(\vec{x} \cdot \vec{y}+\theta)^{d} \\
& K(\vec{x}, \vec{y})=\exp \left(-|\vec{x}-\vec{y}|^{2} / 2 \sigma^{2}\right) \quad \text { Gaussian, } \\
& K(\vec{x}, \vec{y})=\tanh (\kappa(\vec{x} \cdot \vec{y})+\theta) \quad \text { Sigmoidal. }
\end{aligned}
$$

It was shown in Ref. [43] that a suitable function kernel must fulfill Mercer's condition

$$
\int K(\vec{x}, \vec{y}) g(\vec{x}) g(\vec{y}) d \vec{x} d \vec{y} \geq 0
$$

for any function $g$ such that $\int g^{2}(\vec{x}) d \vec{x}$ is finite. While Gaussian and polynomial kernels are known to comply with Mercer's condition, this is not strictly the case for sigmoidal kernels. To extend the linear methodology to nonlinear problems one substitutes $\vec{x}_{i} \cdot \vec{x}_{j}$ by $K\left(\vec{x}_{i}, \vec{x}_{j}\right)$ in Eq. (71). Due to Mercer's conditions on the kernel, the corresponding optimisation problem is a well defined convex quadratic programming problem with a global minimum. This is an advantage of SVMs compared to neural networks where local minima occur.

For regression problems, the same algorithm is used as for classification with the exception that instead of dividing events based on their type (signal/background), it separates them based on the value (larger/smaller than average). In the end, it does not return the sigmoid of the distance between the event and the hyperplane, but the distance itself - increased by the average target value.

\section{Implementation}

The TMVA implementation of the Support Vector Machine follows closely the description given in the literature. It employs a sequential minimal optimisation (SMO) [45] to solve the quadratic problem. Acceleration of the minimisation is achieved by dividing a set of vectors into smaller subsets [46]. The number of training subsets is controlled by the user. The SMO method drives the subset selection to the extreme by selecting subsets of two vectors (for details see Ref. [44]). The pairs of vectors are chosen, using heuristic rules, to achieve the largest possible improvement (minimisation) per step. Because the working set is of size two, it is straightforward to write down the analytical solution. The minimisation procedure is repeated recursively until the minimum is found. The SMO algorithm has proven to be significantly faster than other methods and has become the most common minimisation method used in SVM implementations. The precision of the minimisation is controlled via a tolerance parameter. The SVM training time can be reduced by increasing the tolerance. Most classification problems should be solved with less then 1000 training iterations.

\section{Performance}

The TMVA SVM algorithm comes with linear, polynomial, Gaussian and sigmoidal kernel functions. With sufficient training statistics, the Gaussian kernel allows to approximate any separating function in the input space. It is crucial for the performance of the SVM to appropriately tune the kernel parameters and the cost parameter $C$. In case of a Gaussian, the kernel is tuned via an optional parameter $\Gamma$, which is related to the width $\sigma$ by $\Gamma=1 /\left(2 \sigma^{2}\right)$. The optimal tuning of these parameters is specific to the problem and must be done by the user.

The SVM training time scales with $n^{2}$, where $n$ is the number of vectors (events) in the training data set. The user is therefore advised to restrict the sample size during the first rough scan of the kernel parameters. Also increasing the minimisation tolerance helps to speed up the training.

SVM is a nonlinear general purpose classification and regression algorithm with a performance similar to neural networks (Sec. 7.10) or to a multidimensional likelihood estimator (Sec. 7.3). 


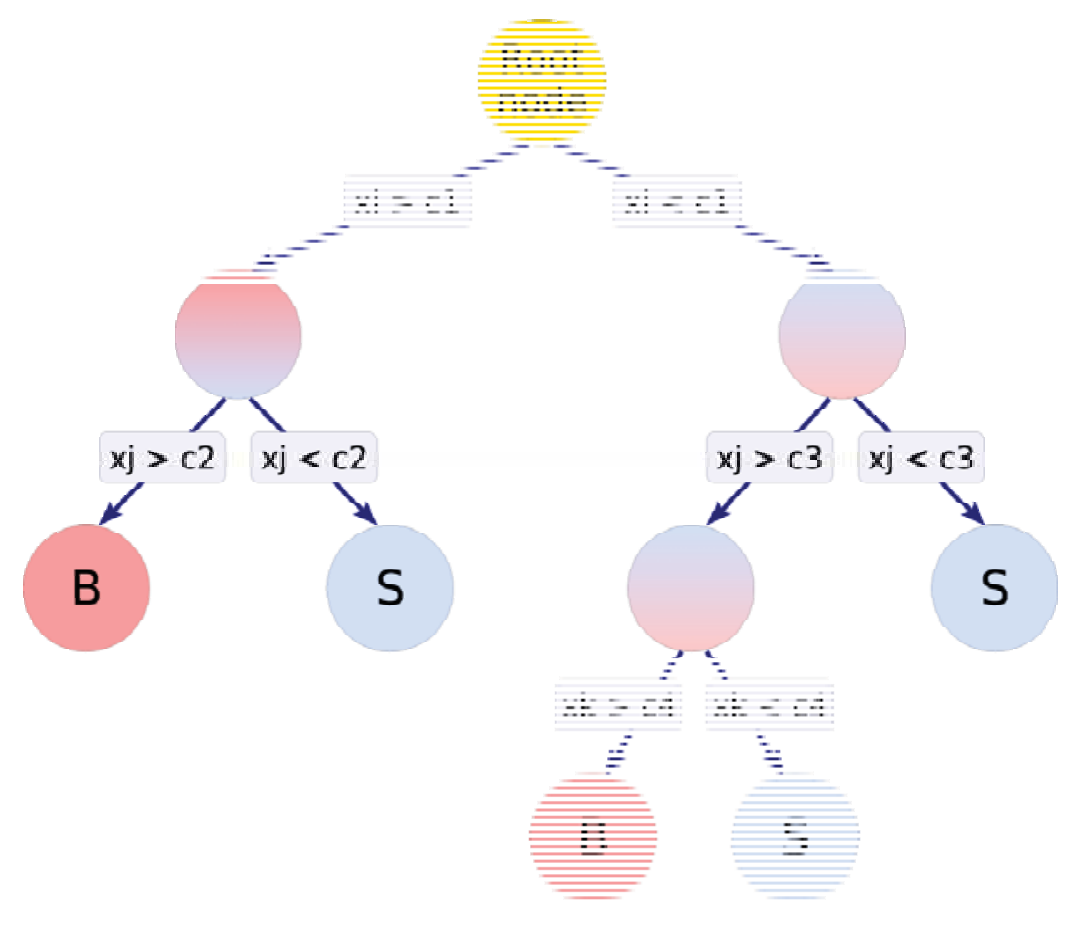

FIG. 16: Schematic view of a decision tree. Starting from the root node, a sequence of binary splits using the discriminating variables $x_{i}$ is applied to the data. Each split uses the variable that at this node gives the best separation between signal and background when being cut on. The same variable may thus be used at several nodes, while others might not be used at all. The leaf nodes at the bottom end of the tree are labeled " $\mathrm{S}$ " for signal and " $\mathrm{B}$ " for background depending on the majority of events that end up in the respective nodes. For regression trees, the node spliting is performed on the variable that gives the maximum decrease in the average squared error when attributing a constant value of the target variable as output of the node, given by the average of the training events in the corresponding (leaf) node (see Sec. 7.12).

\subsection{Boosted Decision and Regression Trees}

A decision (regression) tree $(\mathrm{BDT})^{21}$ is a binary tree structured classifier (regressor) similar to the one sketched in Fig. 16. Repeated left/right (yes/no) decisions are taken on one single variable at a time until a stop criterion is fulfilled. The phase space is split this way into many regions that are eventually classified as signal or background, depending on the majority of training events that end up in the final leaf node. In case of regression trees, each output node represents a specific value of the target variable. ${ }^{22}$ The boosting (see Sec. 6) of a decision (regression) tree extends this concept from one tree to several trees which form a forest. The trees are derived from the same training ensemble by reweighting events, and are finally combined into a single classifier (regressor) which is given by a (weighted) average of the individual decision (regression) trees. Boosting stabilizes the response of the decision trees with respect to fluctuations in the training sample and is able to considerably enhance the performance w.r.t. a single tree. In the following, we will use the term decision tree for both, decision- and regression trees and we refer to regression trees only if both types are treated differently.

Decision trees are well known classifiers that allow a straightforward interpretation as they can be visualized by a simple two-dimensional tree structure. They are in this respect similar to rectangular cuts. However, whereas a cut-based analysis is able to select only one hypercube as region of phase space, the decision tree is able to split the phase space into a large number of hypercubes, each of which is identified as either "signal-like" or "background-like", or attributed a constant event (target) value in case of a regression tree. For classification trees, the path down the tree to each leaf node represents an individual cut sequence that selects signal or background depending on the type of the leaf node.

A shortcoming of decision trees is their instability with respect to statistical fluctuations in the training sample from which the tree structure is derived. For example, if two input variables exhibit similar

\footnotetext{
${ }^{21}$ We use the acronym BDT for decision as well as regression trees.

${ }^{22}$ The target variable is the variable the regression "function" is trying to estimatc.
} 
separation power, a fluctuation in the training sample may cause the tree growing algorithm to decide to split on one variable, while the other variable could have been selected without that fluctuation. In such a case the whole tree structure is altered below this node, possibly resulting also in a substantially different classifier response.

This problem is overcome by constructing a forest of decision trees and classifying an event on a majority vote of the classifications done by each tree in the forest. All trees in the forest are derived from the same training sample, with the events being subsequently subjected to so-called boosting (see Sec. 6), a procedure which modifies their weights in the sample. Boosting increases the statistical stability of the classifier and typically also improves the separation performance compared to a single decision tree. However, the advantage of the straightforward interpretation of the decision tree is lost. While one can of course still look at a limited number of trees trying to interpret the training result, one will hardly be able to do so for hundreds of trees in a forest. Nevertheless, the general structure of the selection can already be understood by looking at a limited number of individual trees. In many cases, the boosting performs best if applied to trees (classifiers) that, taken individually, have not much classification power, i.e. small trees.

\section{Boosting, Bagging and Randomising}

The different "boosting" algorithms (in the following we will call also bagging or randomised trees "boosted") available for decision trees in TMVA are currently :

- AdaBoost (see Sec. 6.1) and AdaBoostR2(see Sec. 26) for regression

- Gradient Boost (see Sec. 6.2) (not for regression)

- Bagging (see Sec. 6.3)

- Randomised Trees, like the Random Forests of L. Breiman [30]. Each tree is grown in such a way that at each split only a random subset of all variables is considered. Moreover, each tree in the forest is grown using only a (resampled) subset of the original training events. The size of the subset as well as the number of variables considered at each split are user-defined.

A possible modification of Eq. (21) for the result of the combined classifier from the forest is to use the training purity ${ }^{23}$ in the leaf node as respective signal or background weights rather than relying on the binary decision. Such an approach however should be adopted with care as the purity in the leaf nodes is sensitive to overtraining and therefore typically overestimated. Tests performed so far with this option did not show significant performance increase. Further studies together with tree pruning are needed to better understand the behaviour of the purity-weighted BDTs.

\section{Training (Building) a decision tree}

The training, building or growing of a decision tree is the process that defines the splitting criteria for each node. The training starts with the root node, where an initial splitting criterion for the full training sample is determined. The split results in two subsets of training events that each go through the same algorithm of determining the next splitting iteration. This procedure is repeated until the whole tree is built. At each node, the split is determined by finding the variable and corresponding cut value that provides the best separation between signal and background. The node splitting stops once it has reached the minimum number of events which is specified in the BDT configuration. The leaf nodes are classified as signal or background according to the class the majority of events belongs to. End-nodes can be classified either in the same way, or according to their purity.

A variety of separation criteria can be configured to assess the performance of a variable and a specific cut requirement. Because a cut that selects predominantly background is as valuable as one that selects signal, the criteria are symmetric with respect to the event classes. All separation criteria have a maximum where the samples are fully mixed, i.e., at purity $p=0.5$, and fall off to zero when the sample consists of one event class only. Tests have revealed no significant performance disparity between the following separation criteria :

- Gini Index [default], defined by $p \cdot(1-p)$;

${ }^{23}$ The purity of a node is given by the ratio of signal events to all events in that node. Hence pure background nodes have zero purity. 
- Cross entropy, defined by $-p \cdot \ln (p)-(1-p) \cdot \ln (1-p)$;

- Misclassification error, defined by $1-\max (p, 1-p)$;

- Statistical significance, defined by $S / \sqrt{S+B}$;

- Average squared error, defined by $1 / N \cdot \sum^{N}(y-\hat{y})^{2}$ for regression trees where $\mathrm{y}$ is the regression target of each event in the node and $\hat{y}$ is its mean value over all events in the node (which would be the estimate of $y$ that is given by the node).

Since the splitting criterion is always a cut on a single variable, the training procedure selects the variable and cut value that optimises the increase in the separation index between the parent node and the sum of the indices of the two daughter nodes, weighted by their relative fraction of events. The cut values are optimised by scanning over the variable range. The default value of 20 scan points proved to be a good compromise between computing time and step size. Finer stepping values did not increase noticeably the performance of the BDTs. However, a truly optimal cut, given the training sample, can be determined by invoking an algorithm that tests all possible cuts on the training sample and finds the best one. This procedure is of course slower than scanning a coarse grid.

In principle, the splitting could continue until each leaf node contains only signal or only background events, which could suggest that perfect discrimination is achievable. However, such a decision tree would be strongly overtrained. To avoid overtraining a decision tree must be pruned.

\section{Pruning a decision tree}

Pruning is the process of cutting back a tree from the bottom up after it has been built to its maximum size. Its purpose is to remove statistically insignificant nodes and thus reduce the overtraining of the tree. It has been found to be beneficial to first grow the tree to its maximum size and then cut back, rather than interrupting the node splitting at an earlier stage. This is because apparently insignificant splits can nevertheless lead to good splits further down the tree. TMVA currently implements two tree pruning algorithms.

- For the expected error pruning [31] ail leaf nodes for which the statistical error estimates of the parent nodes are smaller than the combined statistical error estimates of their daughter nodes are recursively deleted. The statistical error estimate of each node is calculated using the binomial error $\sqrt{p \cdot(1-p) / N}$, where $N$ is the number of training events in the node and $p$ its purity. The amount of pruning is controlled by multiplying the error estimate by the fudge factor (the pruning strength). Expected error pruning is not available for the regression trees.

- Cost complexity pruning [32] relates the number of nodes in a subtree below a node to the gain in terms of misclassified training events by the subtree compared the the node itself with no further splitting. The cost estimate $R$ chosen for the misclassification of training events is given by the misclassification rate $1-\max (p, 1-p)$ in a node. The cost complexity for this node is then defined by

$$
\rho=\frac{R(\text { node })-R(\text { subtree below that node })}{\# \text { nodes(subtree below that node })-1} .
$$

The node with the smallest $\rho$ value in the tree is recursively pruned away as long as $\rho<$ PruneStrength. While for classification trees, one typically uses just the misclassification error in the pruning, but Gini-Index for the node splitting, regression trees use in both cases the squared error loss.

Note that the pruning is performed after the boosting so that the error fraction used by AdaBoost is derived from the unpruned tree.

It is possible to let an algorithm search for the optimal strength parameter. The training sample is divided into two subsamples, of which only one is used for training, while the other one serves for validation. The tree is pruned sequentially starting from the node which has the smallest value of the cost-complexity in the tree. After each pruning step the performance of the tree is assessed using the validation sample. This process is repeated until the ROOT node would be pruned. As optimal prune strength for this tree the value is chose which corresponds to the best performing tree using the validation sample.

While this type of pruning obviously gives the "optimally pruned tree" given the training data, it is not completely clear yet if this also applies for the tree in the forest. Currently it looks as if in TMVA, 
better results for the whole forest are often achieved when pruning is not applied, but rather the maximal tree depth is set to a relatively small value ( 3 or 4 ) already during the tree building phase.

Note that the Gradient boost does not apply a pruning algorithm. In this case it is recommended that the number of nodes in the tree is restricted to values between 5 to 20 .

\section{Variable ranking}

A ranking of the BDT input variables is derived by counting how often the variables are used to split decision tree nodes, and by weighting each split occurrence by the separation gain-squared it has achieved and by the number of events in the node [32]. This measure of the variable importance can be used for a single decision tree as well as for a forest.

\section{Performance}

Only limited experience has been gained so far with boosted decision trees in HEP. In the literature decision trees are sometimes referred to as the best "out of the box" classifiers. This is because little tuning is required in order to obtain reasonably good results. This is due to the simplicity of the method where each training step (node splitting) involves only a one-dimensional cut optimisation. Decision trees are also insensitive to the inclusion of poorly discriminating input variables. While for artificial neural networks it is typically more difficult to deal with such additional variables, the decision tree training algorithm will basically ignore non-discriminating variables as for each node splitting only the best discriminating variable is used. However, the simplicity of decision trees has the drawback that their theoretically best performance on a given problem is generally inferior to other techniques like neural networks. This is seen for example using the academic training samples included in the TMVA package. For this sample, which has equal RMS but shifted mean values for signal and background and linear correlations between the variables only, the Fisher discriminant provides theoretically optimal discrimination results. While the artificial neural networks are able to reproduce this optimal selection performance the BDTs always fall short in doing so. However, in other academic examples with more complex correlations or real life examples, the BDTs often outperform the other techniques. This is because either there are not enough training events available that would be needed by the other classifiers, or the optimal configuration (i.e. how many hidden layers, which variables) of the neural network has not been specified. We have only very limited experience at the time with the regression, hence cannot really comment on the performance in this case.

\subsection{Predictive learning via rule ensembles (RuleFit)}

This classifier is a TMVA implementation of Friedman-Popescu's RuleFit method described in [34]. Its idea is to use an ensemble of so-called rules to create a scoring function with good classification power. Each rule $r_{i}$ is defined by a sequence of cuts, such as

$$
\begin{aligned}
& r_{1}(\mathbf{x})=I\left(x_{2}<100.0\right) \cdot I\left(x_{3}>35.0\right) \\
& r_{2}(\mathbf{x})=I\left(0.45<x_{4}<1.00\right) \cdot I\left(x_{1}>150.0\right) . \\
& r_{3}(\mathbf{x})=I\left(x_{3}<11.00\right)
\end{aligned}
$$

where the $x_{i}$ are discriminating input variables, and $I(\ldots)$ returns the truth of its argument. A rule applied on a given event is non-zero only if all of its cuts are satisfied, in which case the rule returns 1.

The easiest way to create an ensemble of rules is to extract it from a forest of decision trees (cf. Sec. 7.12). Every node in a tree (except the root node) corresponds to a sequence of cuts required to reach the node from the root node, and can be regarded as a rule. Hence for the tree illustrated in Fig. 16 on page 42 a total of 8 rules can be formed. Linear combinations of the rules in the ensemble are created with coefficients (rule weights) calculated using a regularised minimisation procedure [35]. The resulting linear combination of all rules defines a score function (see below) which provides the RuleFit response $y_{\mathrm{RF}}(\mathbf{x})$.

In some cases a very large rule ensemble is required to obtain a competitive discrimination between signal and background. A particularly difficult situation is when the true (but unknown) scoring function 
is described by a linear combination of the input variables. In such cases, e.g., a Fisher discriminant would perform well. To ease the rule optimisation task, a linear combination of the input variables is added to the model. The minimisation procedure will then select the appropriate coefficients for the rules and the linear terms.

As for all TMVA classifiers, the goal of the rule learning is to find a classification function $y_{\mathrm{RF}}(\mathbf{x})$ that optimally classifies an event according to the tuple of input observations (variables) $\mathbf{x}$. The classification function is written as

$$
y_{\mathrm{RF}}(\mathbf{x})=a_{0}+\sum_{m=1}^{M_{R}} a_{m} f_{m}(\mathbf{x}),
$$

where the set $\left\{f_{m}(\mathrm{x})\right\}_{M_{R}}$ forms an ensemble of base learners with $M_{R}$ elements. A base learner may be any discriminating function derived from the training data. In our case, they consist of rules and linear terms as described in the introduction. The complete model then reads

$$
y_{\mathrm{RF}}(\mathbf{x})=a_{0}+\sum_{m=1}^{M_{R}} a_{m} r_{m}(\mathbf{x})+\sum_{i=1}^{n_{\mathrm{var}}} b_{i} x_{i}
$$

To protect against outliers, the variables in the linear terms are modified to

$$
x_{i}^{\prime}=\min \left(\delta_{i}^{+}, \max \left(\delta_{i}^{-}\right)\right),
$$

where $\delta_{i}^{ \pm}$are the lower and upper $\beta$ quantiles ${ }^{24}$ of the variable $x_{i}$. The value of $\beta$ is user-defined. If the variables are used "as is", they may have an unequal a priori influence relative to the rules. To counter this effect, the variables are normalised

$$
x_{i}^{\prime} \rightarrow \sigma_{r} \cdot x_{i}^{\prime} / \sigma_{i},
$$

where $\sigma_{r}$ and $\sigma_{i}$ are the estimated standard deviations of an ensemble of rules and the variable $x_{i}^{\prime}$, respectively.

\section{Rule generation}

The rules are extracted from a forest of decision trees. There are several ways to generate a forest. In the current RuleFit implementation, each tree is generated using a fraction of the training sample. The fraction depends on which method is used for generating the forest. Currently two methods are supported: AdaBoost and Random Forest. For AdaBoost, the whole training set is used for all trees. The diversity is obtained through using different event weights for each tree. Instead, for a random forest, the diversity is created by training each tree using random sub-samples. If this method is chosen, the fraction is calculated from the training sample size $N$ (signal and background) using the empirical formula [36]

$$
f=\min (0.5,(100.0+6.0 \cdot \sqrt{N}) / N) .
$$

By default, AdaBoost is used for creation of the forest. In general it seems to perform better than the random forest.

The topology of each tree is controlled by the minimum and maximum number of events allowed for further splitting of a node. For each tree, a fraction is drawn from a uniform distribution within the given range. The obtained fraction is then multiplied with the number of training events used for the tree, giving the minimum number of events in a node to allow for splitting. In this way both large trees (small fraction) giving complex rules and small trees (large fraction) for simple rules are created. For a given forest of $N_{t}$ trees, where each tree has $n_{\ell}$ leaf nodes, the maximum number of possible rules is

$$
M_{R, \max }=\sum_{i=1}^{N_{t}} 2\left(n_{\ell, i}-1\right) .
$$

\footnotetext{
${ }^{24}$ Quantiles are points taken at regular intervals from the PDF of a random variable. For example, the 0.5 quantile corresponds to the median of the PDF.
} 
To prune similar rules, a distance is defined between two topologically equal rules. Two rules are topologically equal if their cut sequences follow the same variables only differing in their cut values. The rule distance used in TMVA is then defined by

$$
\delta_{R}^{2}=\sum_{i} \frac{\delta_{i, L}^{2}+\delta_{i, U}^{2}}{\sigma_{i}^{2}},
$$

where $\delta_{i, L(U)}$ is the difference in lower (upper) limit between the two cuts containing the variable $x_{i}$ : $i=1, \ldots, n_{\mathrm{var}}$. The difference is normalised to the RMS-squared $\sigma_{i}^{2}$ of the variable. Similar rules with a distance smaller than a user-defined minimum are removed from the rule ensemble. This parameter can be tuned to improve speed and to suppress noise. In principle, this should be achieved in the fitting procedure. However, pruning the rule ensemble using a distance cut will reduce the fitting time and will probably also reduce the number of rules in the final model. Note that the cut should be used with care since a too large cut value will deplete the rule ensemble and weaken its classification performance.

\section{Fitting}

Once the rules are defined, the coefficients in Eq. (79) are fitted using the training data. For details, the fitting method is described in [35]. A brief description is provided below to motivate the corresponding RuleFit options.

A loss function $L\left(y_{\mathrm{RF}}(\mathbf{x}) \mid \hat{y}\right)$, given by the "squared-error ramp" [35]

$$
L\left(y_{\mathrm{RH}} \mid \hat{y}\right)=\left(\hat{y}-H\left(y_{\mathrm{RH}}\right)\right)^{2},
$$

where $H(y)=\max \left(-1, \min \left(y_{\mathrm{R} F}, 1\right)\right)$, quantifies the "cost" of misclassifying an event of given true class $\hat{y}$. The risk $R$ is defined by the expectation value of $L$ with respect to $\mathrm{x}$ and the true class. Since the true distributions are generally not known, the average of $N$ training events is used as an estimate

$$
R=\frac{1}{N} \sum_{i=1}^{N} L\left(y_{\mathrm{RF}}\left(\mathbf{x}_{i}\right) \mid \hat{y}_{i}\right) .
$$

A line element in the parameter space of the rule weights (given by the vector $\mathbf{a}$ of all coefficients) is then defined by

$$
\mathbf{a}(\epsilon+\delta \epsilon)=\mathbf{a}(\epsilon)+\delta \epsilon \cdot \mathbf{g}(\epsilon),
$$

where $\delta \epsilon$ is a positive small increment and $\mathbf{g}(\epsilon)$ is the negative derivative of the estimated risk $R$, evaluated at $\mathbf{a}(\epsilon)$. The estimated risk-gradient is evaluated using a sub-sample of the training events.

Starting with all weights set to zero, the consecutive application of Eq. (87) creates a path in the a space. At each step, the procedure selects only the gradients $g_{k}$ with absolute values greater than a certain fraction $(\tau)$ of the largest gradient. The fraction $\tau$ is an a priori unknown quantity between 0 and 1 . With $\tau=0$ all gradients will be used at each step, while only the strongest gradient is selected for $\tau=1$. A measure of the "error" at each step is calculated by evaluating the risk (Eq. 86) using the validation sub-sample. By construction, the risk will always decrease at each step. IIowever, for the validation sample the value will increase once the model starts to be overtrained.

If the selected $\tau$ is a negative number, the best value is estimated by means of a scan. In such a case several paths are fitted in parallel, each with a different value of $\tau$. The number of paths created depend on the required precision on $\tau$. By only selecting the paths being "close enough" to the minimum at each step, the speed for the scan is kept down. The path leading to the lowest estimated error is then selected. Once the best $\tau$ is found, the fitting proceeds until a minimum is found. A simple example with a few scan points is illustrated in Fig. 17. 


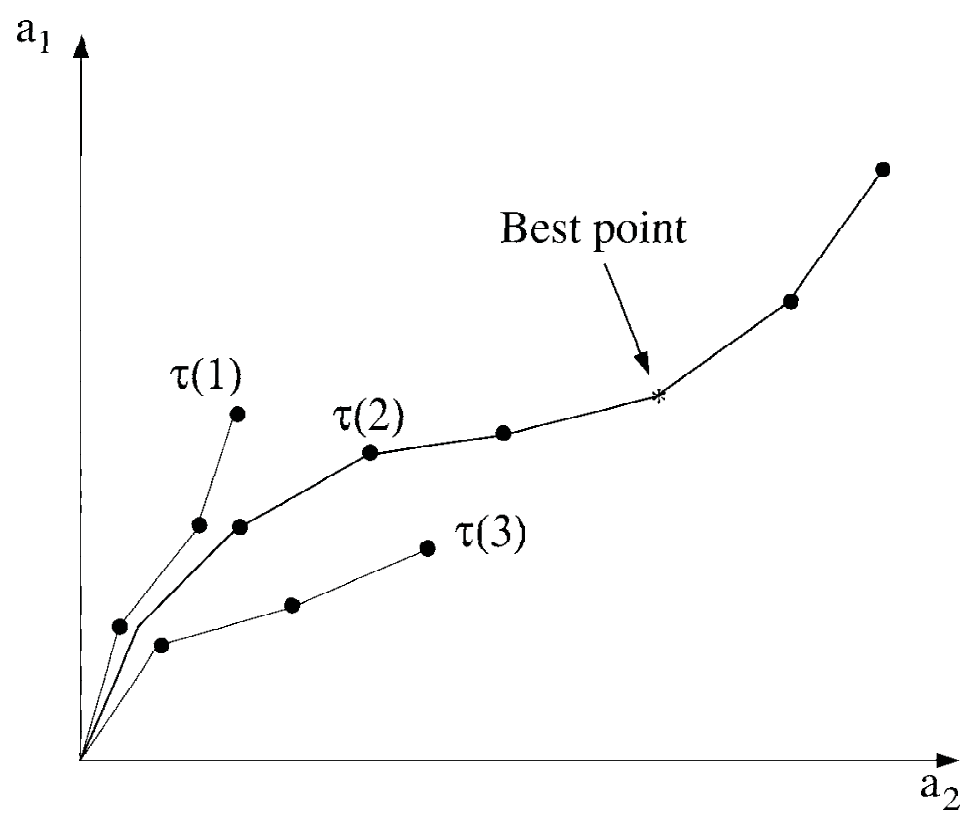

Fig. 17: An example of a path scan in two dimensions. Each point represents an $\epsilon$ in Eq. (87) and each step is given by $\delta \epsilon$. The direction along the path at each point is given by the vector $\mathrm{g}$. For the first few points, the paths $\tau(1,2,3)$ are created with different values of $\tau$. After a given number of steps, the best path is chosen and the search is continued. It stops when the best point is found. That is, when the estimated error-rate is minimum.

\subsubsection{Variable ranking}

Since the input variables are normalised, the ranking of variables follows naturally from the coefficients of the model. To each rule $m\left(m=1, \ldots, M_{R}\right)$ can be assigned an importance defined by

$$
I_{m}=\left\{a_{m} \mid \sqrt{s_{m}\left(1.0-s_{m}\right)},\right.
$$

where $s_{m}$ is the support of the rule with the following definition

$$
s_{m}=\frac{1}{N} \sum_{n=1}^{N} r_{m}\left(\mathbf{x}_{n}\right) .
$$

The support is thus the average response for a given rule on the data sample. A large support implies that many events pass the cuts of the rule. Hence, such rules cannot have strong discriminating power. On the other hand, rules with small support only accept few events. They may be important for these few events they accept, but they are not in the overall picture. The definition (88) for the rule importance suppresses rules with both large and small support.

For the linear terms, the definition of importance is

$$
I_{i}=\left|b_{i}\right| \cdot \sigma_{i},
$$

so that variables with small overall variation will be assigned a small importance.

A measure of the variable importance may then be defined by

$$
J_{i}=I_{i}+\sum_{m \mid x_{i} \in r_{m}} I_{m} / q_{m},
$$

where the sum is over all rules containing the variable $x_{i}$, and $q_{m}$ is the number of variables used in the rule $r_{m}$. This is introduced in order to share the importance equally between all variables in rules with more than one variable. 


\section{Performance}

Rule ensemble based learning machines are not yet well known within the HEP community, although they start to receive some attention [37]. Apart from RuleFit [34] other rule ensemble learners exists, such as SLIPPER [38].

The TMVA implementation of RuleFit follows closely the original design described in Ref. [34]. Currently the performance is however slightly less robust than the one of the Friedman-Popescu package. Also, the experience using the method is still scarce at the time of this writing.

To optimise the performance of RuleFit several strategies can be employed. The training consists of two steps, rule generation and rule ensemble fitting. One approach is to modify the complexity of the generated rule ensemble by changing either the number of trees in the forest, or the complexity of each tree. In general, large tree ensembles with varying trees sizes perform better than short non-complex ones. The drawback is of course that fitting becomes slow. However, if the fitting performs well, it is likely that a large amount of rules will have small or zero coefficients. These can be removed, thus simplifying the ensemble. The fitting performance can be improved by increasing the number of steps along with using a smaller step size. Again, this will be at the cost of speed performance although only at the training stage. The setting for the parameter $\tau$ may greatly affect the result. Currently an automatic scan is performed by default. In general, it should find the optimum $\tau$. If in doubt, the user may set the value explicitly. In any case, the user is initially advised to use the automatic scan option to derive the best path.

\section{Combining MVA Methods}

In challenging classification or regression problems with a high demand for optimisation, or when treating feature spaces with strongly varying properties, it can be useful to combined MVA methods. There is large room for creativity inherent in such combinations. For TMVA we distinguish three classes of combinations:

1. Boosting MVA methods,

2. Categorising MVA methods,

3. Building committees of MVA methods.

While the first two combined methods are available since TMVA 4.1, the third one is still under development. The MVA booster is a generalisation of the Boosted Decision Trees approach (see Sec. 7.12) to any method in TMVA. Category methods allow the user to specify sub-regions of phase space, which are assigned by requirements on input or spectator variables, and which define disjoint sub-populations of the training sample, thus improving the modelling of the training data. Finally, Committee methods allow one to input MVA methods into other MVA methods, a procedure that can be arbitrarily chained.

All of these combined methods are of course MVA methods themselves, treated just like any other method in TMVA for training, testing, evaluation and application. This also allows to categorise a committee method, for example.

\subsection{Boosted classifiers}

Since generalised boosting is not yet available for regression in TMVA, we restrict the following discussion to classification applications. A boosted classifier is a combination of a collection of classifiers of the same type trained on the same sample but with different events weights. ${ }^{25}$ The response of the final classifier is a weighted response of each individual classifier in the collection. The boosted classifier is potentially more powerful and more stable with respect to statistical fluctuations in the training sample. The latter is particularly the case for bagging as "boost" algorithm (cf.Sec. 6.3, page 21).

The following sections do not apply to decision trees. We refer to Sec. 7.12 (page 42) for a description of boosted decision trees. In the current version of TMVA only the AdaBoost and Bagging algorithms are implemented for the boost of arbitrary classifiers. The boost algorithms are described in detail in Sec. 6 on page 19 .

\footnotetext{
${ }^{25}$ The Boost method is at the moment only applicable to classification problems.
} 
The parameters most relevant for the boost process are the number of boost iterations and the choice of the boost algorithm (several options). By default boosted classifiers are combined as a weighted average with weights computed from the misclassification error. It is also possible to use the arithmetic average instead.

The boosting process was originally introduced for simple classifiers. The most commonly boosted classifier is the decision tree (DT - cf.Sec. 7.12, page 42). Decision trees need to be boosted a few hundred times to effectively stabilise the BDT response and achieve optimal performance.

Another simple classifier in the TMVA package is the Fisher discriminant (cf.Sec. 7.7, page $32-$ which is equivalent to the linear discriminant described in Sec. 7.8). Because the output of a Fisher discriminant represents a linear combination of the input variables, a linear combination of different Fisher discriminants is again a Fisher discriminant. Hence linear boosting cannot improve the performance. It is nevertheless possible to effectively boost a linear discriminant by applying the linear combination not on the discriminant's output, but on the actual classification results provided. ${ }^{26}$ This corresponds to a "non-linear" transformation of the Fisher discriminant output according to a step function. The Boost method in TMVA also features a fully non-linear transformation that is directly applied to the classifier response value. Overall, the following transformations are available :

- linear : no transformation is applied to the MVA output.

- step : the output is -1 below the step and +1 above (default setting),

- log : logarithmic transformation of the output.

We point out that the performance of a boosted classifier strongly depends on its characteristics as well as on the nature of the input data. A careful adjustment of options is required if AdaBoost is applied to an arbitrary classifier, since otherwise it might even lead to a worse performance than for the unboosted method.

\subsection{Category Classifier}

The Category method allows the user to separate the training data (and accordingly the application data) into disjoint sub-populations exhibiting different properties. The separation into phase space regions is done by applying requirements on the input and/or spectator variables (variables defined as spectators are not used as direct inputs to MVA methods). It thus reaches beyond a simple sub-classification of the original feature space. In each of these disjoint regions (each event must belong to one and only one region), an independent training is performed using the most appropriate MVA method, training options and set of training variables in that zone. The division into categories in presence of distinct subpopulations reduces the correlations between the training variables, improves the modelling; and hence increases the classification and regression performance. ${ }^{27}$ Another common application is when variables are not available in some phase space regions. For example, a sub-detector may only cover a fraction of the training data sample. By defining two categories the variables provided by this detector could be excluded from the training in the phase space region outside of its fiducial volume. Finally, an advantage of categorisation, often exploited in likelihood fits, also lies in the gain of signal significance when the fraction of signal events in the event sample differs between the categories.

We point out that the explicit use of the Category method is not mandatory. The categorisation functionality can be achieved by hand by training independent MVAs in each of the disjoint regions. Our experience however shows that often the corresponding bookkeeping is considered too cumbersome and the required categorisation is not performed, thus leading to performance loss. ${ }^{28}$ The Category method is straightforward to implement and should help in almost all analyses to better describe the training sample. Performance comparison with the corresponding non-categorised method should guide the user in his/her choice.

\footnotetext{
26 Note that in the TMVA standard cxample, which uses lincarly corrclated, Gaussian-distributcd input variables for signal and background, a single Fisher discriminant, already provides the theoretically maximum separation power. Hence on this example, no further gain can be expected by boosting, no matter what, "tricks" are applied.

${ }_{27}$ This is particularly the case for projective likelihood methods, even when including prior decorrelation of the input data (cf. Sec. 7.2).

${ }^{28}$ In the above example with the reduced detector fiducial acceptance, it often occurs that non-physical values ("- $\left.99 "\right)$ are assigned to the variables for events where they are not available. Inserting such meaningless (apart from indicating the category) values into an MVA penalises its performance.
} 
For the current release, the Category method is only implemented for classification. Extension to regression is however straightforward and should follow soon.

The following example illustrates how sub-classifiers are added to a MethodCategory object 'mcategory' :

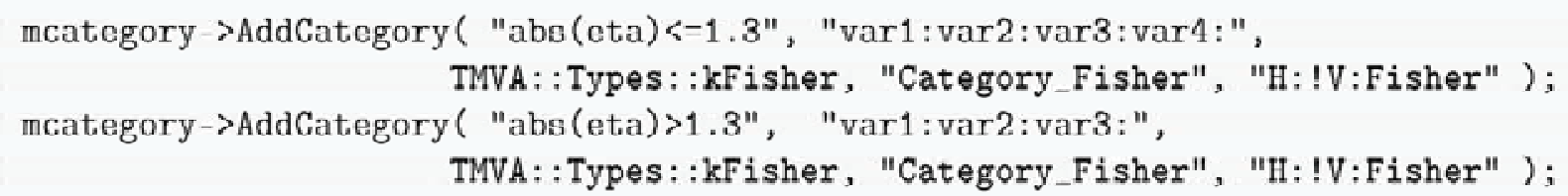

Code Example 3: Adding a category with sub-classifier of type Fisher : the cut in the first argument defines the region to which each of the sub-classifiers are applied, the second argument is the list of variables which are to be considered for this region (in this example var4 is not available when abs (eta) $>1.3$ ), the third argument contains the internal enumerator for a classifier of type Fisher (the MVA method may differ between categories, the fourth argument is the user-defined identifier, and the fifth argument contains the options string for the Fisher classifier. The variable eta must have been defined as an input or spectator variable.

The Category method is implemented in an entirely transparent way, conserving full flexibility for the user on which input variables, MVA method and options to use in each category, and how the category should be defined.

The categories are defined via cuts on the input and/or spectator variables. In case of particularly intricate category definitions it may turn out advantageous for the user to pre-define and pre-fill category variables, and use those as spectators in TMVA to set an event's category. It is required that the cuts are defined in such a way that they create disjoint event samples. TMVA will issue a fatal error in case of an ambiguous category definition.

The advantage offered by the Category classifier is illustrated with the following academic example. We consider four uncorrelated and Gaussian distributed input variables for training. The mean values of the variables are shifted between signal and background, hence providing classification information. In addition, all mean values (signal and background) depend on a spectator variable eta : for abs (eta) $>1.3$ $($ abs $($ eta $)<=1.3)$ they have a positive (negative) offset. Hence, the Gaussians are broader and the variables receive an effective correlation when integrating over eta. The distributions for signal and background of one out of the four variables is shown for both abs (eta) categories in Fig. 18. Figure 19 shows the ROC curves of the categorised and non-categorised Fisher and Projective Likelihood classifiers. Optimum performance is recovered by the Category classifier.

\section{Performance}

The performance of the Category method depends entirely on the judicious choice of the categories, properly separating events with different properties, and on the performance of the sub-methods within these categories. Control by performance comparison with the corresponding non-categorised methods allows to measure the performance gain, and to assess whether categorisation improves the classification.

\section{Which MVA method should I use for my problem?}

There is obviously no generally valid answer to this question. To guide the user, we have attempted a coarse assessment of various MVA properties in Table 5. Simplicity is a virtue, but only if it is not at the expense of significant loss of discrimination power. Robustness with respect to overtraining could become an issue when the training sample is scarce. Some methods require more attention than others in this regard. For example, boosted decision trees are particularly vulnerable to overtraining if used without care. $^{29}$ To circumvent overtraining a problem-specific adjustment of the pruning strength parameter is required.

\footnotetext{
${ }^{29}$ However, experience shows that the BDT performance is amazingly robust - even for strongly overtrained decision trees.
} 


\section{TMVA Input Variables: var4}

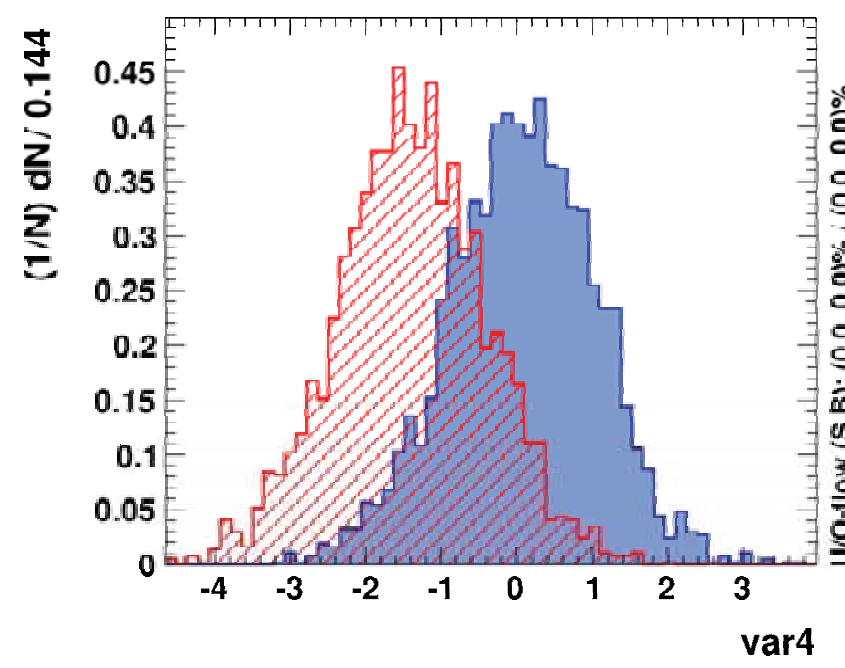

\section{TMVA Input Variables: var4}

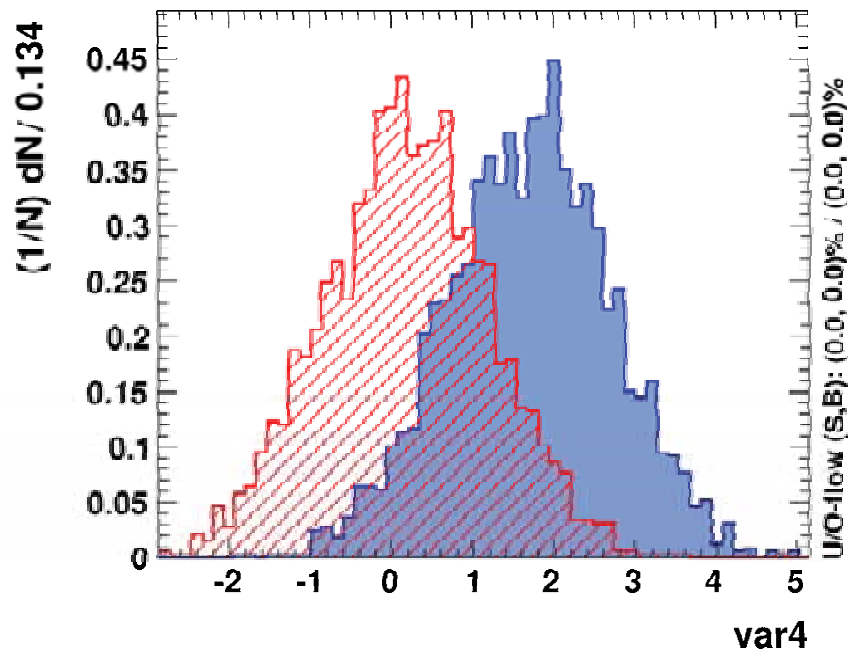

FIG. 18: Examples for the usage of categories. The variable var4 depends on abs (eta) : for abs (eta) $<=1.3$ (left plot), the signal and background Gaussian distributions are both shifted to lower values, while for abs (eta) $>1.3$ (right plot) shifts to larger values have been applied. Ignoring this dependence, broadens the inclusive Gaussian distributions and generates variable correlations (here a coefficient of +0.4 between var 3 and var 4 ) due to the existence of distinct subsamples. The Category classifier treats the two cases independently, thus recovering optimal separation.

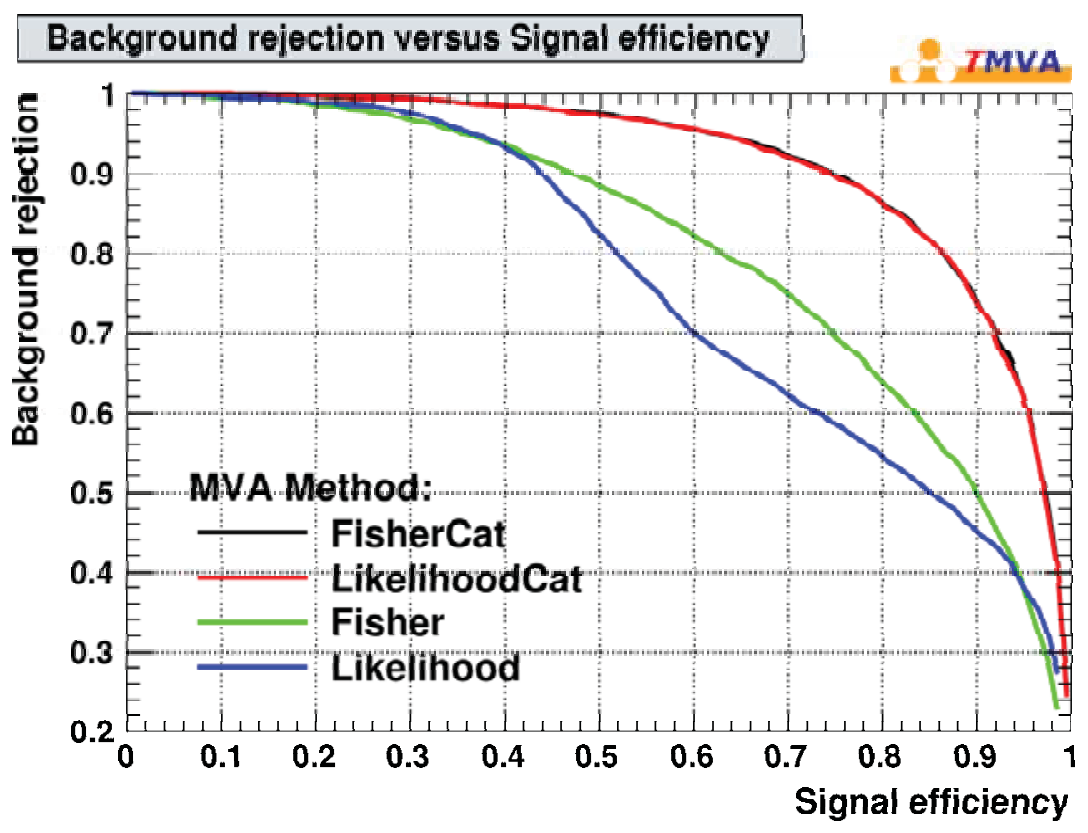

FIG. 19: ROC curves for categorised and non-categorised Fisher and Projective Likelihood classifiers applied to an academic four-Gaussian example with eta-dependent mean values distinguishing two categories (cf. Fig. 18 for the category-dependent signal and background distributions of one of the four input variables). The categorised ("...Cat") classifiers recover optimum performance (their red and black curves are superimposed). 


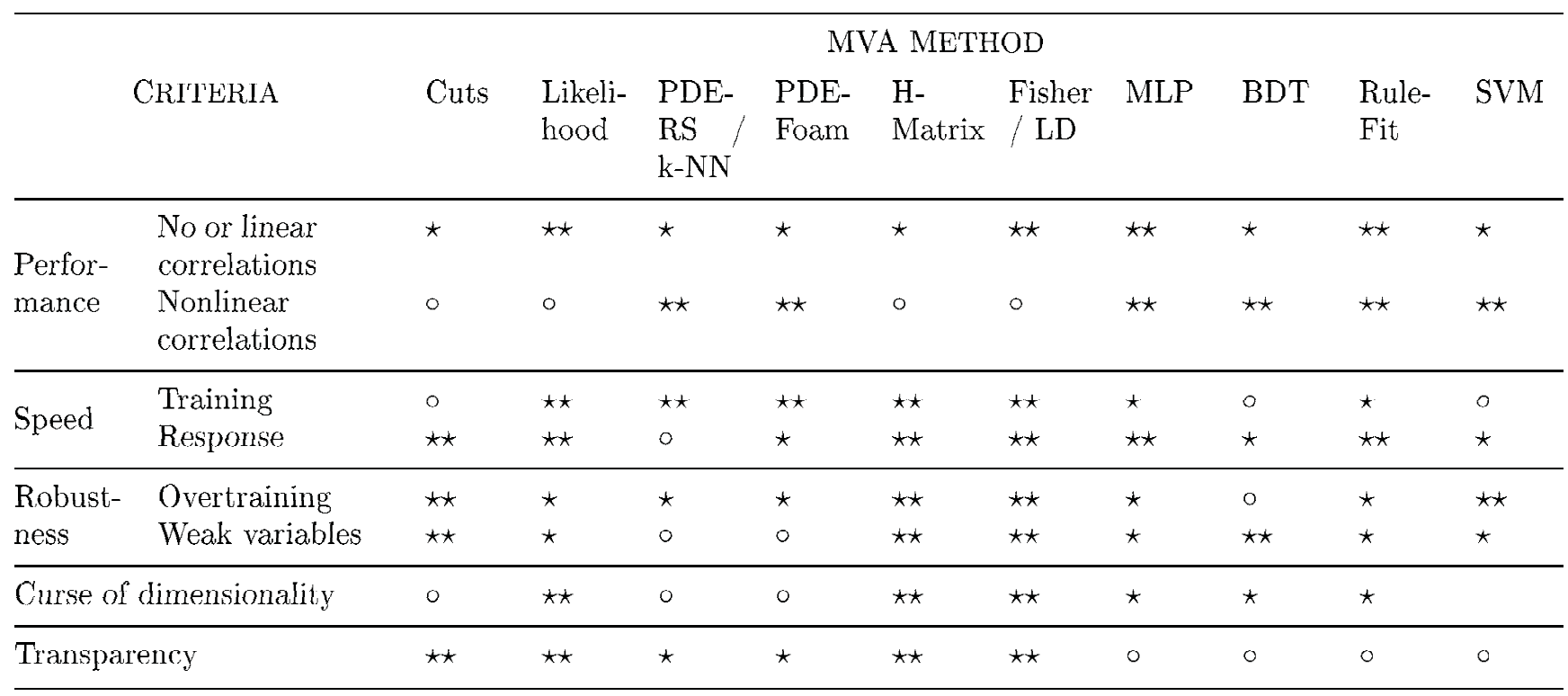

TAB. 5: Assessment of MVA method properties. The symbols stand for the attributes "good" ( $\star \star$ ), "fair" ( $\star$ ) and "bad" (o). "Curse of dimensionality" refers to the "burden" of required increase in training statistics and processing time when adding more input variables. See also comments in the text. The FDA method is not listed here since its properties depend on the chosen function.

To assess whether a linear discriminant analysis (LDA) could be sufficient for a classification (regression) problem, the user is advised to analyse the correlations among the discriminating variables (among the variables and regression target) by inspecting scatter and profile plots (it is not enough to print the correlation coefficients, which by definition are linear only). Using an LDA greatly reduces the number of parameters to be adjusted and hence allow smaller training samples. It usually is robust with respect to generalisation to larger data samples. For moderately intricate problems, the function discriminant analysis (FDA) with some added nonlinearity may be found sufficient. It is always useful to cross-check its performance against several of the sophisticated nonlinear methods to see how much can be gained over the use of the simple and very transparent FDA.

For problems that require a high degree of optimisation and allow to use a large number of input variables, complex nonlinear methods like neural networks, the support vector machine, boosted decision trees and/or RuleFit are more appropriate.

Very involved multi-dimensional variable correlations with strong nonlinearities are usually best mapped by the multidimensional probability density estimators such as PDE-RS, k-NN and PDE-Foam, requiring however a reasonably low number of input variables.

For RuleFit classification we emphasise that the TMVA implementation differs from Friedman-Popescu's original code [34], with slightly better robustness and out-of-the-box performance for the latter version. In particular, the behaviour of the original code with respect to nonlinear correlations and the curse of dimensionality would have merited two stars. ${ }^{30}$ We also point out that the excellent performance for by majority linearly correlated input variables is achieved somewhat artificially by adding a Fisher-like term to the RuleFit classifier (this is the case for both implementations, cf. Sec. 7.13 on page 45).

In Fig 7 (page 15) we have shown the example of ROC-curves obtained from various linear and nonlinear classifiers on a simple toy Monte Carlo sample of linear correlated Gaussian distribution functions. Here all classifiers capable of dealing with such type of correlations are equally performing. Only the projective likelihood method, which ignores all correlations, performs significantly worse (it recovers optimum performance after prior decorrelation of the variables). For such a problem, a Fisher (aka linear) discriminant is optimal by construction, and the non-linear methods effectively reduce in the training to linear discriminants achieving competitive, but somewhat less robust performance.

For another academic toy Monte Carlo example however, which exhibits strongly non-linear correlations in form of a "Schachbrett" (chess board with two-dimensional Gaussian-distributed signal and

${ }^{30}$ An interface to Fricdman-Popescu's original code can be requested from the TMVA authors. 

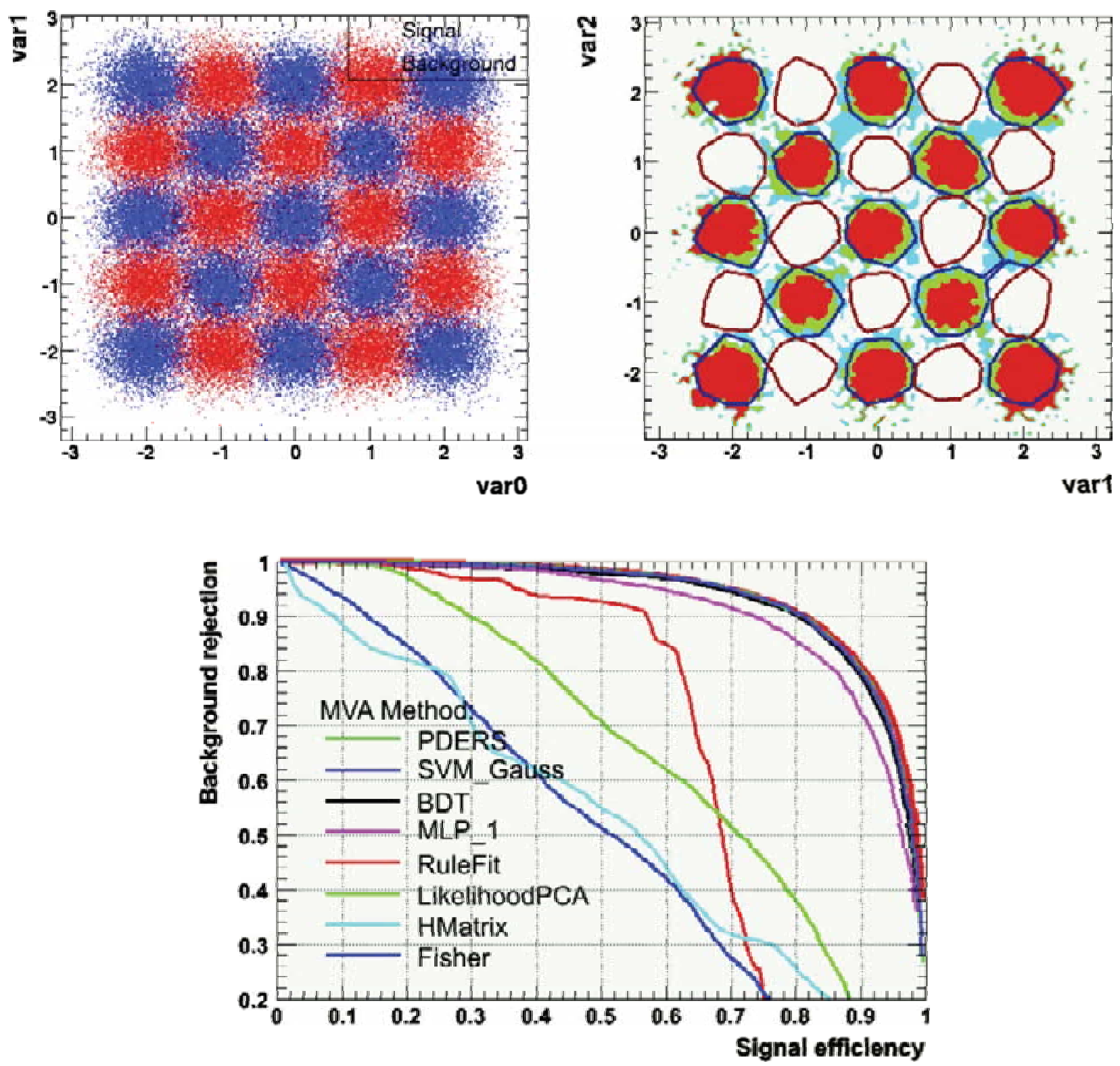

FIG. 20: The top left plot shows the two-dimensional Gaussian-distributed signal (blue) and background (red) distributions for the "Schachbrett" toy Monte Carlo sample. In the top right plot is drawn as an example the Support Vector Machine's classification response in terms of the weights given to events in the two dimensional plane. Dark red signifies large MVA output values, i.e. signal like event regions. The bottom plot gives the ROC curves obtained with several TMVA classifiers for this example after training. The theoretically best ROC curve is known and shown by the thick red outer line. While all the non-linear classification methods (after carefully tuning their parameters) are able to approximate the theoretical limit, the linear methods strongly underperform. 
background spots - see Fig. 20), the limitations of the linear classifiers, being unable to capture the features of the data, become manifest. Non-linear classifiers, once appropriately optimised such as choosing a sufficiently complex network architecture for the MLP, or the proper kernel functions in the SVM, all non-linear methods reproduce or approximate the theoretical limit of this classification problem (indicated by the thick red line in the ROCc curve in the lower plot of Fig. 20).

\section{TMVA implementation status summary for classification and re- gression}

All TMVA methods are fully operational for user analysis, requiring training, testing, evaluating and reading for the application to unknown data samples. Additional features are optional and - despite our attempts to provide a fully transparent analysis - not yet uniformly available. A status summary is given in Table 6 and annotated below.

Although since TMVA 4 the framework supports multi-dimensional MVA outputs it has not yet been implemented for classification. For regression, only a few methods are fully multi-target capable so far (see Table 6).

Individual event-weight support is now commonly realised, only missing (and not foreseen to be provided) for the less recommended neural network CFMlpANN. Support of negative event weights occurring; e.g., in NLO MC requires more scrutiny.

Ranking of the input variables cannot be defined in a straightforward manner for all MVA methods. Transparent and objective variable ranking through performance comparison of the MVA method under successive elimination of one input variable at a time is under consideration (so far only realised for the naive-Bayes likelihood classifier).

Standalone $\mathrm{C}++$ response classes (not required when using the Reader application) are generated by the majority of the classifiers, but not yet for regression analysis. The missing ones for PDE-RS, PDE-Foam, k-NN, Cuts and CFMipANN will only be considered on explicit request.

The availability of help messages, which assist the user with the performance tuning and which are printed on standard output when using the booking option ' $\mathrm{H}$ ', is complete.

Finally, custom macros are provided for some MVA methods to analyse specific properties, such as the fidelity of likelihood reference distributions or the neural network architecture, etc. More macros can be added upon user request.

\section{Conclusions and Plans}

TMVA is a toolkit that unifies highly customisable multivariate (MVA) classification and regression algorithms in a single framework thus ensuring convenient use and an objective performance assessment. It is designed for machine learning applications in high-energy physics, but not restricted to these. Source code and library of TMVA-v.3.5.0 and higher versions are part of the standard ROOT distribution kit (v5.14 and higher). The newest TMVA development version can be downloaded from Sourceforge.net at http://tmva.sourceforge.net.

This Users Guide introduced the main steps of a TMVA analysis allowing a user to optimise and perform her/his own multivariate classification or regression. Let us recall the main features of the TMVA design and purpose :

- TMVA works in transparent factory mode to allow an unbiased performance assessment and comparison : all MVA methods see the same training and test data, and are evaluated following the same prescription.

- A complete TMVA analysis consists of two steps :

1. Training : the ensemble of available and optimally customised MVA methods are trained and tested on independent signal and background data samples; the methods are evaluated and the most appropriate (performing and concise) ones are selected.

2. Application : selected trained MVA methods are used for the classification of data samples with unknown signal and background composition, or for the estimate of unknown target values 


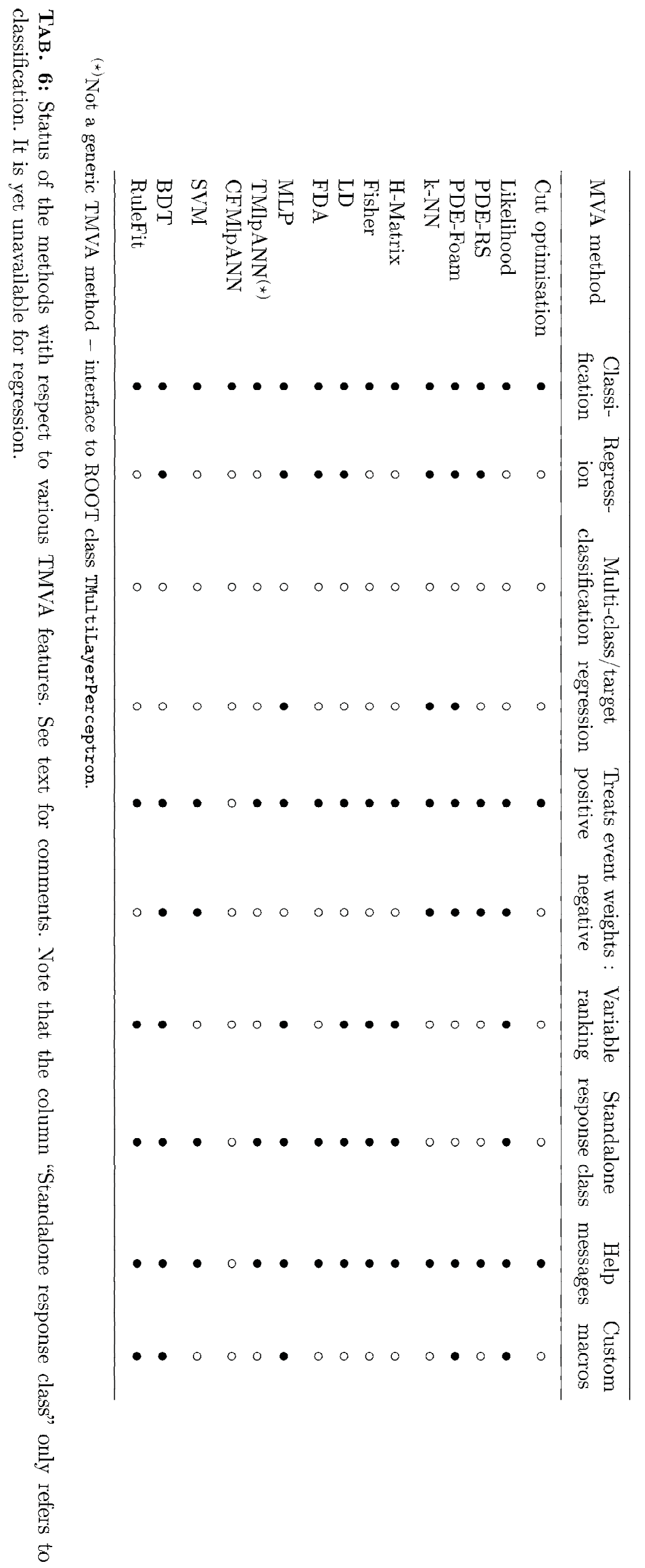


(regression).

- A Factory class object created by the user organises the customisation and interaction with the MVA methods for the training, testing and evaluation phases of the TMVA analysis. The training results together with the configuration of the methods are written to result ("weight") files in XML format.

- Standardised outputs during the Factory running, and dedicated ROOT macros allow a refined assessment of each method's behaviour and performance for classification and regression.

- Once appropriate methods have been chosen by the user, they can be applied to data samples with unknown classification or target values. Here, the interaction with the methods occurs through a Reader class object created by the user. A method is booked by giving the path to its weight file resulting from the training stage. Then, inside the user's event loop, the MVA response is returned by the Reader for each of the booked MVA method, as a function of the event values of the discriminating variables used as input for the classifiers. Alternatively, for classification, the user may request from the Reader the probability that a given event belongs to the signal hypothesis and/or the event's Rarity.

- In parallel to the XML files, TMVA generates standalone C++ classes after the training; which can be used for classification problems (feature not available yet for regression). Such classes are available for all classifiers except for cut optimisation, PDE-RS, PDE-Foam, k-NN and the old CFMlpANN.

We give below a summary of the TMVA methods, outlining the current state of their implementation, their advantages and shortcomings.

- Rectangular Cut Optimisation

The current implementation is mature. It includes speed-optimised range searches using binary trees, and three optimisation algorithms : Monte Carlo sampling, a Genetic Algorithm and Simulated Annealing. In spite of these tools, optimising the cuts for a large number of discriminating variables remains challenging. The user is advised to reduce the available dimensions to the most significant variables (e.g., using a principal component analysis) prior to optimising the cuts.

- Likelihood

Automatic non-parametric probability density function (PDF) estimation through histogram smoothing and interpolation with various spline functions and quasi-unbinned kernel density estimators is implemented. The PDF description can be individually tuned for each input variable.

- PDE-RS

The multidimensional probability density estimator ( $P D E)$ approach is in an advanced development stage featuring adaptive range search, several kernel estimation methods, and speed optimised range search using event sorting in binary trees. It has also been extended to regression.

- PDE-Foam

This new multidimensional PDE algorithm uses self-adapting phase-space binning and is a fast realisation of PDE-RS in fixed volumes, which are determined and optimised during the training phase. Much work went into the development of PDE-Foam. It has been thoroughly tested, and can be considered a mature method. PDE-Foam performs classification and regression analyses.

$-k-N N$

The k-Nearest Neighbour classifier is also in a mature state, featuring both classification and regression. The code has been well tested and shows satisfactory results. With scarce training statistics it may slightly underperform in comparison with PDE-RS, whereas it is significantly faster in the application to large data samples.

- Fisher and H-Matrix

Both are mature algorithms, featuring linear discrimination for classification only. Higher-order correlations are taken care of by FDA (see below).

- Linear Discriminant (LD)

$\mathrm{LD}$ is equivalent to Fisher but providing both classification and linear regression.

- Function Discriminant Analysis (FDA)

FDA is a mature algorithm, which has not been extensively used yet. It extends the linear discriminant to moderately non-linear correlations that are fit to the training data.

- Artificial Neural Networks 
Significant work went into the implementation of fast feed-forward multilayer perceptron algorithms into TMVA. Two external ANNs have been integrated as fully independent methods, and another one has been newly developed for TMVA, with emphasis on flexibility and speed. The performance of the latter ANN (MLP) has been cross checked against the Stuttgart ANN (using as an example $\tau$ identification in ATLAS), and was found to achieve competitive performance. The MLP ANN also performs multi-target regression.

- Support Vector Machine

SVM is a relatively new multivariate analysis algorithm with a strong statistical background. It performs well for nonlinear discrimination and is insensitive to overtraining. Optimisation is straightforward due to a low number of adjustable parameters (only two in the case of Gaussian kernel). The response speed is slower than for a not-too-exhaustive neural network, but comparable with other nonlinear methods. SVM is being extended to multivariate regression.

- Boosted Decision Trees

The BDT implementation has received constant attention over the years of its development. The current version includes additional features like bagging or gradient boosting, and manual or automatic pruning of statistically insignificant nodes. It is a highly performing MVA method that also applies to regression problems.

- RuleFit

The current version has the possibility to run either the original program written by J. Friedman [34] or an independent TMVA implementation. The TMVA version has been improved both in speed and performance and achieves almost equivalent results with respect to the original one, requiring however somewhat more tuning.

The new framework introduced with TMVA 4 provides the flexibility to combine MVA methods in a general fashion. Exploiting these capabilities for classification and regression however requires to create socalled committee methods for each combination. So far, we provide a generalised Boost method, allowing to boost any classifier by simply setting the variable Boost_Num in the configuration options to a positive number (plus possible adjustment of other configuration parameters). The result is a potentially powerful committee method unifying the excellent properties of boosting with MVA methods that already represent highly optimised algorithms.

Boosting is not the only combination the new framework allows us to establish. TMVA now also supports the categorisation of classification according to the phase space, which allows a better modelling and hence simplifies the mapping of the feature space. This particularly improves simple methods, such las projective likelihood and linear discriminants.

It is also planned to develop a committee method that allows to insert the result of MVA methods as input to another MVA method. 


\section{Acknowledgements}

We thank our colleagues and friends from the TMVA core developer group Peter Speckmayer, Jörg Stelzer, Jan Therhaag and Eckhard von Töerne for the fruitful collaboration. The fast growth of TMVA would not have been possible without the contribution and feedback from many developers (also coauthors of this Users Guide) and users to whom we are indebted. We thank in particular the CERN Summer students Matt Jachowski (Stanford U.) for the implementation of TMVA's MLP neural network, and Yair Mahalalel (Tel Aviv U.) for a significant improvement of PDE-RS. The Support Vector Machine has been contributed to TMVA by Andrzej Zemla and Marcin Wolter (IFJ PAN Krakow), and the kNN method has been written by Rustem Ospanov (Texas U.). We are grateful to Lucian Ancu, Doug Applegate, Kregg Arms, Renè Brun and the ROOT team, Andrea Bulgarelli, Marc Escalier, Zhiyi Liu, Colin Mclean, Elzbieta Richter-Was, Alfio Rizzo, Lydia Roos, Vincent Tisserand, Alexei Volk, Jiahang Zhong for helpful feedback and bug reports. Thanks also to Lucian Ancu for improving the plotting macros.

\section{Références}

[1] J. Neyman and E. Pearson, "On the Problem of the Most Efficient Tests of Statistical Hypotheses". Philosophical Transactions of the Royal Society of London, Series A, Containing Papers of a Mathematical or Physical Character 231, 289 (1933); P.G. Hoel, S.C. Port, and C.J. Stone, "Testing Hypotheses", Ch. 3 in Introduction to Statistical Theory. New York, Houghton Mifflin, 56, 1971.

[2] R. Brun and F. Rademakers, "ROOT - An Object Oriented Data Analysis Framework", Nucl. Inst. Meth. in Phys. Res. A 389, 81 (1997).

[3] J. Friedman, T. Hastie and R. Tibshirani, "The Elements of Statistical Learning", Springer Series in Statistics, 2001.

[4] A. Webb, "Statistical Pattern Recognition", 2nd Edition, J. Wiley \& Sons Ltd, 2002.

[5] L.I. Kuncheva, "Combining Pattern Classifiers", J. Wiley \& Sons, 2004.

[6] A. Hoecker et al., "The TMVA Users Guide", arXiv :physics/0703039, version 5 July 2009.

[7] I. Narsky, "StatPatternRecognition : A $C++$ Package for Statistical Analysis of High Energy Physics Data", physics/0507143 (2005).

[8] The following web pages give information on available statistical tools in HEP and other areas of science : https://plone4.fnal.gov:4430/P0/phystat/, http://astrostatistics.psu.edu/statcodes/.

[9] The BABAR Physics Book, BABAR Collaboration (P.F. Harrison and H. Quinn (editors) et al.), SLAC-R-0504 (1998); S. Versillé, PhD Thesis at LPNHE, http://pnhe-babar.in2p3.fr/theses/these_ SophieVersille.ps.gz (1998).

[10] To our information, the Rarity has been originally defined by F. Le Diberder in an unpublished Mark II internal note. In a single dimension, as defined in Eq. (13), it is equivalent to the $\mu$-transform developed in : M. Pivk, "Etude de la violation de $C P$ dans la désintégration $B^{0} \rightarrow h^{+} h^{-}(h=\pi, K)$ auprès du détecteur BABAR à SLAC', PhD thesis (in French), http://tel.archives-ouvertes.fr/documents/archives0/00/00/29/91/index_fr.html (2003).

[11] M. Kendall, A. Stuart, K.J. Ord, and S. Arnold, Kendall's Advanced Theory of Statistics : Volume 2A - Classical Inference and and the Linear Model (Kendall's Library of Statistics), A Hodder Arnold Publication, $6^{\text {th }}$ edition, April 1999.

[12] T.M. Cover and J.A. Thomas, "Elements of information theory", Wiley-Interscience, New York, NY, USA, 1991.

[13] Y.-I. Moon, B. Rajagopalan, and U. Lall, Phys. Rev. E, 52 (no. 3), 2318 (1995).

[14] J.L. Bentley, "Multidimensional Binary Search Trees Used for Associate Searching", Communications of the ACM, 18, 509 (1975) ; J.L. Bentley, "Multidimensional Divide-and-Conquer", Communications of the ACM, 23(4), 214 (1980); J.H. Friedman, J.L. Bentley and R.A. Finkel, "An Algorithm for Finding Matches in Logarithmic Expected Time", ACM Trans. on Mathematical Software, 3(3), 209 (1977). 
[15] R. Sedgewick, "Algorithms in C++", Addison Wesley, Chapter 26, Boston, USA (1992).

[16] T. Carli and B. Koblitz, Nucl. Instrum. Meth. A 501, 576 (2003) [hep-ex/0211019].

[17] S. Jadach, Comput. Phys. Commun. 130, 244 (2000); S. Jadach, Comput. Phys. Commun. 152, 55 (2003).

[18] D. Dannheim et al., Nucl. Instr. and Meth. A 606, 717 (2009).

[19] F. James, "MINUIT, Function Minization and ERROR Analysis", Version 94.1, CERN program Library long writeup D506.

[20] P.J.M. Van Laarhoven and E.H.L. Aart, "Simulated Annealing : Theory and Application", D. Reidel Publishing, Dordrecht, Holland, 1987.

[21] N. Metropolis, A.W. Rosenbluth, M.N. Rosenbluth, A.M. Teller, and E. Teller, J. Chem. Phys. 21, $6,1087(1953)$.

[22] 353QH twice smoothing algorithm, presented by J. Friedman in Proc. of the 1974 CERN School of Computing, Norway, Aug 11-24, 1974.

[23] D.W. Scott, "Multivariate Density Estimation, Theory, Practice, and Visualization", WileyInterscience, New York, 1992.

[24] R.A. Fisher, Annals Eugenics 7, 179 (1936).

[25] P.C. Mahalanobis, Proc. Nat. Inst. Sci. India, Part 2A, 49 (1936); P.C. Mahalanobis, "On the generalized distance in statistics", Proceedings of the National Institute of Science, Calcutta, 12, 49 (1936).

[26] C.G. Broyden, "The Convergence of a Class of Double-rank Minimization Algorithms", J. Inst. of Math. and App. 6, 76 (1970); R. Fletcher, "A New Approach to Variable Metric Algorithms", Computer J. 13, 317 (1970) ; D. Goldfarb, "A Family of Variable Metric Updates Derived by Variational Means", Math. Comp. 24, 23 (1970); D.F. Shannon, "Conditioning of Quasi-Newton Methods for Function Minimization", Math. Comp. 24, 647 (1970).

[27] R.E. Schapire, "The strenght of weak learnability, (1990) Machine Learning 5 197-227; Y. Freud, "Boosting a weak learning algorithm by majority (1995) Inform. and Comput. 121 256-285;

[28] Y. Freund and R.E. Schapire, J. of Computer and System Science 55, 119 (1997).

[29] H. Drucker,Improving regressors using boosting techniques,In D. H. Fisher (Ed.), Proceedings of the fourteenth international conference on machine learning (ICML 1997) (pp. 107i $\measuredangle \frac{1}{2} 115$ ). Nashville. TN, USA, July $8 \ddot{i} ; \frac{1}{2} 12$. Morgan Kaufmann, ISBN $1 \ddot{i} ; \frac{1}{2} 55860 i ; \frac{1}{2} 486 i i \frac{1}{2} 3$.

[30] L. Breiman, Random Forests, Technical Report, University of California 2001.

[31] Y.R. Quinlan, "Simplifying Decision Trees", Int. J. Man-Machine Studies, 28, 221 (1987).

[32] L. Breiman, J. Friedman, R. Olshen and C. Stone, "Classification and Regression Trees", Wadsworth (1984).

[33] We use the ROOT class TParallelCoord by B. Dalla Piazza, 2007.

[34] J. Friedman and B.E. Popescu, "Predictive Learning via Rule Ensembles", Technical Report, Statistics Department, Stanford University, 2004.

[35] J. Friedman and B.E. Popescu, "Gradient Directed Regularization for Linear Regression and Classification", Technical Report, Statistics Department, Stanford University, 2003.

[36] RuleFit web site : http:/www-stat.stanford.edu/ jhf/r-rulefit/RuleFit_help.html.

[37] J. Conrad and F. Tegenfeldt, JHEP 0607, 040 (2006) [hep-ph/0605106].

[38] W. Cohen and Y. Singer, Proceedings of the Sixteenth National Conference on Artificial Intelligence (AAAI-99), 335, AAAI Press, 1999.

[39] V. Vapnik and A. Lerner, "Pattern recognition using generalized portrait method", Automation and Remote Control, 24, 774 (1963).

[40] V. Vapnik and A. Chervonenkis, "A note on one class of perceptrons", Automation and Remote Control, 25 (1964). 
[41] B.E. Boser, I.M. Guyon, and V.N. Vapnik, "A training algorithm for optimal margin classifiers", in D. Haussler, ed., Proceedings of the 5th Annual ACM Workshop on Computational Leraning Theory, 144, ACM Press (1992).

[42] C. Cortes and V. Vapnik, "Support vector networks", Machine Learning, 20, 273 (1995).

[43] V. Vapnik, "The Nature of Statistical Learning Theory", Springer Verlag, New York, 1995.

[44] C.J.C. Burges, "A Tutorial on Support Vector Machines for Pattern Recognition", Data Mining and Knowledge Discovery, 2, 1 (1998).

[45] J. Platt, "Fast training of support vector machines using sequential minimal optimization", in B. Scholkopf, C. Burges and A. Smola, eds., Advances in Kernel Methods - Support Vector Learning, ch. 12, pp. 185, MIT Press, 1999.

[46] S. Keerthi, S. Shevade, C. Bhattacharyya and K. Murthy, "Improvements to Platt's SMO algorithm for SVM classifier design", Technical Report CD-99-14, Dept. of Mechanical and Production Engineering, Natl. Univ. Singapore, Singapore, 1999.

[47] P. Huber, "Robust estimation of a location parameter", Annals of Mathematical Statistics, 35, 73101,1964 